\title{
A rigorous definition of fiberwise quantum cohomology and equivariant quantum cohomology
}

\author{
Peng LU
}

\section{Introduction.}

The notion of quantum cohomology was first proposed by the physicist Vafa [V] and its mathematical foundation was established by Ruan and Tian [RT] for semi-positive symplectic manifolds. However computing the quantum cohomology is a difficult task in general since it does not have the functorial property of behaving well under pull-back. If $X$ and $Y$ are two semi-positive symplectic manifolds, and $f: X \rightarrow Y$ is a continuous map, then there is a ring homomorphism $f^{*}: H^{*}(Y ; \mathbb{Q}) \rightarrow H^{*}(X ; \mathbb{Q})$ for ordinary cohomology. However for quantum cohomology, there does not exist such homomorphism preserving quantum multiplication. The quantum cohomology of projective spaces is known for quite some time. The quantum cohomology of Grassmannians was computed by Witten [W] and by Seibert and Tian [ST]. The notion of equivariant quantum cohomology was first proposed by Givental and Kim in a heuristic way [GK], and they conjectured several properties of equivariant quantum cohomology, namely direct product, restriction and induction. Assuming these properties, they computed the quantum cohomology of complete flag manifolds rigorously. Later, another account was given by Astashkevish and Sadov [AS]. They also introduced the notion of fiberwise quantum cohomology heuristically (called vertical quantum cohomology in [AS]), and computed the quantum cohomology of partial flag manifolds again assuming the conjectured properties. Kim did a similar thing for partial flag manifolds [K1]. The advantage of fiberwise quantum cohomology and equivariant quantum cohomology is that the functorial pull-back property of ordinary cohomology is partially restored for them, i.e. restriction property. It is exactly this property and several other functorial properties that enables Givental et al. to complete the computation. Ciocan-Fontanine computed the Gromov-Witten invariants of complete flag manifolds using the canonical complex structure, so he could give a presentation of the quantum cohomology of complete flag 
manifolds rigorously $[\mathrm{CF}]$. Other examples of quantum cohomology were worked out by Batyrev for toric manifolds [B].

In this work we will give a mathematical rigorous definition of fiberwise quantum cohomology and equivariant quantum cohomology in strong semi-positive symplectic case(see definition below). Assume that $\mathcal{X} \rightarrow Y$ is a smooth fiber bundle, $Y$ is a closed smooth manifold and the fiber are closed manifolds. Further we assume that $\mathcal{X}$ admits a differential two form $\omega$ whose restriction to each fiber $V_{z}$ is a strong semi-positive symplectic form. The main results of this paper are: 1) We will define fiberwise GromovWitten mixed invariants (called fiberwise mixed invariants below) of arbitrary genus for $\mathcal{X} \rightarrow Y$ using perturbed pseudo-holomorphic curves, then we prove several properties of fiberwise mixed invariants such as direct product, restriction, induction and the so-called composition law; 2) Using fiberwise mixed invariants, we will define fiberwise quantum cohomology and equivariant quantum cohomology. The properties alluded to above namely direct product, restriction, induction and associativity of fiberwise quantum cohomology and equivariant quantum cohomology will then follow from the corresponding properties of the fiberwise mixed invariants. So we put the computation in [GK], [AS] and [K1] on a solid foundation; 3) As examples, we will compute the fiberwise quantum cohomology of several fiber bundles with fiber a flag manifold. In this paper, we basically will follow the approach of Ruan and Tian to quantum cohomology [RT]. The main part of this paper will be devoted to a proof of the composition law of the fiberwise mixed invariants. In the proof, most analytic results are adopted from [RT].

This paper is organized as following: Section 1 contains a compactness theorem and several transversality result for the moduli space we used to define the fiberwise mixed invariants. The definition of the fiberwise mixed invariants and some simple properties are given in section 2. Section 3 is devoted to establish the composition law of the fiberwise mixed invariants. Section 4 contains the definitions of fiberwise quantum cohomology and equivariant quantum cohomology, their properties are proved. In section 5 , fiberwise quantum cohomologies of several fiber bundles with fiber being a flag manifold are computed.

During the preparation of this paper, the author learned B. Kim's paper "On equivariant quantum cohomology" [K2], in which he defined equivariant quantum cohomology for flag manifolds only. His approach was different from ours; he used Kontsevich's moduli space of stable maps to define equivariant Gromov-Witten classes. 
Acknowledgment. This paper is part of the author's dissertation at SUNY, Stony Brook. I am extremely grateful to my advisor Prof. Gang Tian for introducing me to the problem and for his patient help. I would like to thank Prof. Blaine H. Lawson Jr. for teaching me the ring structures of partial flag manifolds.

\section{A Compactness Theorem and Several Transversality Results.}

\subsection{A Compactness Theorem.}

Definition 1.1. (i) An almost complex structure $J$ on a symplectic manifold $(V, \omega)$ is said to be $\omega$-tamed if $\omega(\xi, J \xi)>0$, for any $\xi \neq 0 \in T V$, and if further $\omega(J \xi, J \eta)=\omega(\xi, \eta)$, for all $\xi, \eta \in T V$, we call $J \omega$-compatible.

(ii) A symplectic manifold $(V, \omega)$ is called strong semi-positive if for any $\omega$-compatible almost complex structure $J$ on $V$, every $J$-holomorphic curve $u: \mathbb{C} P^{1} \rightarrow V$ has non-negative first Chern number, i.e. $\int_{P^{1}} u^{*} c_{1}(T V) \geq 0$. If this inequality is always strictly positive, we call $(V, \omega)$ positive symplectic manifold.

Remark 1.1. The concept of semi-positive symplectic manifolds was originally defined by $\mathrm{D}$. McDuff in [M3]. That definition requires that there is no spherical second homology class $A$ such that $\omega(A)>0,0>c_{1}(A) \geq 3-n$. We will need above definition when we show the dimension of boundaries of the moduli space is codimension 2 in section 2 . Note both monotone symplectic manifolds and Calabi-Yau manifolds are strong semi-positive. The author thanks the referee for pointing out this difference.

The following is the basic setup we will work with in this section.

Assumption ( $\star$ ). Let $\mathcal{X} \stackrel{p}{\rightarrow} Y$ be a smooth fiber bundle over a closed smooth manifold $Y$ whose fiber are closed manifolds diffeomorphic to $V$ of dimension $2 n$. Assume that $\mathcal{X}$ admits a differential two form $\omega$ with bounded $H^{l, q}$ Sobolev norm $(l \geq 10, q>1)$, whose restriction to each fiber $V_{z}$ is a symplectic form. Here $V_{z}$ is the fiber over a point $z \in Y$, i.e. $\omega$ is a fiberwise symplectic form on $\mathcal{X}$. Assume further that $\left(V_{z},\left.\omega\right|_{V_{z}}\right)$ is a strong semi-positive symplectic manifold for each $z$ in $Y$. 
To define the fiberwise quantum cohomology for $\mathcal{X} \rightarrow Y$, we first need to define the fiberwise mixed invariants. Suppose that $\Sigma$ is a stable curve of genus $g$ with $m$ irreducible components $\Sigma_{i}$ and $k$ marked points. Assume that $A_{i} \in H_{2}(V ; \mathbb{Z})$ are $m$ homology classes. The fiberwise mixed invariant can be considered as a multilinear map

$$
\Phi_{\left(A_{1}, \cdots, A_{m}, \omega, \Sigma, P\right)}^{v}: \prod_{1}^{k} H^{*}(\mathcal{X}, \mathbb{Q}) \times \prod_{1}^{l} H^{*}(\mathcal{X}, \mathbb{Q}) \rightarrow H^{*}(Y, \mathbb{Q}) .
$$

To evaluate the image of this map on the element $\gamma \in H_{*}(Y, \mathbb{Q})$, a key observation is that we should use the pull-back pseudo-manifold fiber bundle $\mathcal{X}_{Z} \rightarrow Z$ where $F: Z \rightarrow Y$ is a pseudo-manifold representative of $\gamma$ and the pseudo-manifold $\mathcal{X}_{Z}$ is the fiber product $Z \times_{F} \mathcal{X}$. The advantage of this definition is that it enables us to prove the restriction property easily. Its price is that $Z$ is only a pseudo-manifold, so that we need to carefully treat the moduli space of each stratum of $Z$.

A compact pseudo-manifold in $Y$ is a stratified space $Z$ together with a continuous map $F: Z \rightarrow Y$ satisfying: each stratum is a smooth manifold without boundary, each lower stratum is at least codimension 2 , and $F$ is smooth on each stratum. Note that any rational homology class in $Y$ can be represented by a finite dimensional compact pseudo-manifold, and any two such pseudo-manifolds representing the same homology class form the boundary of a compact pseudo-manifold (and so are cobordant in the usual sense) (see [C]). Fix a finite dimensional compact pseudo-manifold $F: Z \rightarrow Y$, and denote by $\mathcal{X}_{Z}$ the fiber product $Z \times_{F} \mathcal{X}$. Then $\mathcal{X}_{Z} \rightarrow Z$ is a pseudo-manifold fibration with fiber $V$. $\omega$ induces a two form on $\mathcal{X}_{Z}$ by the natural map $\mathcal{X}_{Z} \stackrel{\tilde{F}}{\rightarrow} \mathcal{X}$, which is still denoted by $\omega$. It is a fiberwise symplectic form on $\mathcal{X}_{Z}$. Note that $\omega$ is continuous and is smooth on each stratum. $\left(\mathcal{X}_{Z}, \omega, Z, V\right)$ is the basic object we will study below.

Remark 1.2. We can define a trivialization of $\mathcal{X}_{Z}$ as follows. For any $z_{\infty} \in Z$, choose a neighborhood $W$ of $F\left(z_{\infty}\right)$ in $Y$, such that $\left.\mathcal{X}\right|_{W} \cong W \times V$. Choose a neighborhood $U$ of $z_{\infty}$ in $Z$ such that $F(U) \subset W$, then $\left.\mathcal{X}_{Z}\right|_{U}$ has an induced trivialization $\left.\mathcal{X}_{Z}\right|_{U} \cong U \times V$, where $U$ may not be smooth. Note that for any two different trivializations of $\mathcal{X}_{Z}$ over $U_{1}$ and $U_{2}$, the transition function $f_{12}: U_{1} \cap U_{2} \rightarrow \operatorname{Diff}(V)$ factors through as $U_{1} \cap U_{2} \stackrel{F}{\rightarrow} W_{1} \cap W_{2} \stackrel{h_{12}}{\longrightarrow}$ $\operatorname{Diff}(V)$, where $h_{12}$ is smooth transition function between two trivializations of $\mathcal{X}$ over $W_{1}$ and $W_{2}$. This property is important for defining various convergences below. This trivialization of $\mathcal{X}_{Z}$ is crucial when we study the structure of moduli space. We fix a such trivialization for $\mathcal{X}_{Z}$ from now on. 
Define a vector bundle $E \stackrel{\pi}{\rightarrow} \mathcal{X}_{Z}$ whose fiber $E_{x}$ is $T_{x} V_{z}$ for each $x \in$ $\mathcal{X}_{Z}, z=p(x)$. A complex structure $J$ on $E$ is called a fiberwise almost complex structure on $\mathcal{X}_{Z}$. A complex structure $J$ on $E$ is said to be tamed by $\omega$ if $\omega(\xi, J \xi)>0$, for any $\xi \neq 0 \in E$. If further $\omega(J \xi, J \eta)=\omega(\xi, \eta)$, for any $\xi, \eta \in E$, we call the complex structure $J$ on $E \omega$-compatible. We now show that there is a fiberwise almost complex structure on $\mathcal{X}_{Z}$ compatible with $\omega$.

Lemma 1.1. Under Assumption( $(*)$, the vector bundle $E$ admits a continuous complex structure which has bounded $H^{l, q}$ norm on each stratum, and is compatible with $\omega$. Furthermore for any $z_{\infty} \in Z, J$ satisfies

Condition 1. $\left\|\left.J\right|_{z_{r} \times V}-\left.J\right|_{z_{\infty} \times V}\right\|_{C^{4}(V)} \rightarrow 0$ as $z_{r} \rightarrow z_{\infty}, z_{r} \in U$. Here we use the trivialization in Remark 1.2 and view $\left.J\right|_{*}$ as a section of $\operatorname{Hom}(T V, T V)$.

Proof. Since the vector bundle $E$ is a pseudo-manifold smooth in fiber directions, we can find a continuous Riemannian metric $g$ on $E$, such that $g$ is smooth on each stratum and $g$ satisfies $\left\|\left.g\right|_{z_{r} \times V}-\left.g\right|_{z_{\infty} \times V}\right\|_{C^{4}(V)} \rightarrow 0$ as $z_{r} \rightarrow z_{\infty}, z_{r} \in U$. Here we use the trivialization in Remark 1.2 and view $\left.g\right|_{*}$ as a section of $\operatorname{Hom}(T V \times T V, \mathbb{R})$. Then there is an unique automorphism $A$ of $E$ such that $\omega(\xi, \eta)=g(A \xi, \eta)$, for any $\xi, \eta \in E_{x}, x \in \mathcal{X}_{Z}$. Since $\omega$ is skew symmetric, $-A^{2}$ is positive definite with respect to $g$. It is easy to check that $J=\left(-A^{2}\right)^{-\frac{1}{2}} A$ is the required complex structure on $E$.

Note that since the deformation of compatible complex structures on the bundle $E$ is un-obstructed, the tangent space at $J$ consists of all sections $s$ of the bundle $E n d(E)$ satisfying $s \circ J+J \circ s=0$ and $g(s \xi, \eta)=g(\xi, s \eta)$ for all $\xi, \eta \in E$. Let $\mathcal{J}_{l, q}^{v}\left(\mathcal{X}_{Z}, \omega\right)$ be the space of $\omega$-compatible continuous complex structures on $E$ with bounded $H^{l, q}$ norm on each stratum satisfying Condition 1. Here and in the following ' $v$ ' means fiberwise. Although $J$ is not smooth, $\mathcal{J}_{l, q}^{v}\left(\mathcal{X}_{Z}, \omega\right)$ is a smooth Banach manifold.

Next we will define the so-called fiberwise perturbed $J$-holomorphic map. Recall that a $k$-point genus $g$ stable curve $C=\left(\Sigma ; x_{1}, \cdots, x_{k}\right)$ is a reduced connected curve $\Sigma$ whose singularities are only double points (it may have no singularities at all), plus $k$ distinct smooth points $x_{1}, \cdots, x_{k}$ in $\Sigma$, such that every smooth rational component of $\Sigma$ contains at least three points from $x_{1}, \cdots, x_{k}$ or double points of $\Sigma$, and every elliptic curve component contains at least one such points. Such a $\Sigma$ is called an admissible curve. 
Assume that $\Sigma$ has $m$ irreducible components $\Sigma_{1}, \cdots, \Sigma_{m}$ (where $\Sigma_{i}$ may have double points). Let $J$ be a fiberwise almost complex structure on $\mathcal{X}_{Z}$, and $\pi_{j}$ be the projection from $\Sigma_{i} \times \mathcal{X}_{Z}$ to its $j$-th factor $(j=1,2)$. A fiberwise inhomogeneous term $\nu$ over $\Sigma \times \mathcal{X}_{Z}$ is a set of sections $\nu_{1}, \cdots, \nu_{m}$, where each $\nu_{i}$ is a section of $\operatorname{Hom}\left(\pi_{1}^{*} T \Sigma_{i}, \pi_{2}^{*} E\right)$, satisfying

(i) $\nu_{i}$ is continuous everywhere and differentiable on each stratum;

(ii) Condition 2. Use the notation of Remark 1.2, for any $z_{\infty} \in Z$,

$$
\left\|\left.\nu_{i}\right|_{\Sigma_{i} \times z_{r} \times V}-\left.\nu_{i}\right|_{\Sigma_{i} \times z_{\infty} \times V}\right\|_{C^{4}\left(\Sigma_{i} \times V\right)} \rightarrow 0, \text { as } z_{r} \rightarrow z_{\infty}, z_{r} \in U ;
$$

(iii) The $J$-anti-linear condition: $\nu_{i}\left(j_{\Sigma_{i}}\left(v_{i}\right)\right)=-J\left(\nu_{i}\left(v_{i}\right)\right)$, where $j_{\Sigma_{i}}$ is the complex structure on $\Sigma_{i}, v_{i}$ is any vector in $T \Sigma_{i}$.

Definition 1.2. Let $\Sigma$ be an admissible curve, $J$ a fiberwise almost complex structure on $\mathcal{X}_{Z}$, and $\nu$ a fiberwise inhomogeneous term on $\Sigma \times \mathcal{X}_{Z}$. A fiberwise $(J, \nu)$-perturbed holomorphic map from $\Sigma$ into $\mathcal{X}_{Z}$ is a continuous map $f: \Sigma \rightarrow \mathcal{X}_{Z}$ which is differentiable at the smooth points of $\Sigma$, such that $f(\Sigma) \subset V_{z}$ for some $z \in Z$, and $f$ satisfies the inhomogeneous CauchyRiemann equation on each component $\Sigma_{i}(i=1, \cdots, m)$. Let $f_{i}=\left.f\right|_{\Sigma_{i}}$. Then

$$
\left(\bar{\partial}_{J} f_{i}\right)(x)=\nu_{i}\left(x, f_{i}(x)\right), \text { for any } x \in \Sigma_{i} \backslash \operatorname{Sing}\left(\Sigma_{i}\right), \quad i=1, \cdots, m,
$$

where $\bar{\partial}_{J}$ denotes the differential operator $d+J \circ d \circ j_{\Sigma_{i}}$. We denote the space of all fiberwise $(J, \nu)$ holomorphic map $f$ from $\Sigma$ into $\mathcal{X}_{Z}$ satisfying $\left[f_{i *}\left(\Sigma_{i}\right)\right]=A_{i}, i=1, \cdots, m$ by $\mathcal{M}_{\left(A_{1}, \cdots, A_{m}\right)}^{v}\left(\Sigma, \mathcal{X}_{Z}, J, \nu\right)$. Here $A_{1}, \cdots, A_{m}$ are $\mathrm{m}$ homology classes in $H_{2}(V, \mathbb{Z})$.

We will use $\mathcal{M}_{\left(A_{1}, \cdots, A_{m}\right)}^{v}\left(\Sigma, \mathcal{X}_{Z}, J, \nu\right)$ to define the fiberwise mixed invariants later. This moduli space is similar to the moduli space used to prove that the Gromov-Witten mixed invariants are independent of the choice of the almost complex structure (see $[R T]$ ). In fact that moduli space is the same as our moduli space for the case where the bundle is $V \times[0,1] \rightarrow[0,1]$. The further generality lies in that the bundle $\mathcal{X}_{Z} \rightarrow Z$ may be nontrivial and that $Z$ is only a pseudo-manifold. Now we begin establishing a compactness theorem about the structure of $\mathcal{M}_{\left(A_{1}, \cdots, A_{m}\right)}^{v}\left(\Sigma, \mathcal{X}_{Z}, J, \nu\right)$ which is required for the definition of the fiberwise mixed invariants.

Recall that a degeneration of admissible curves is a holomorphic fibration $\tilde{\pi}: S \rightarrow \Delta \subset \mathbb{C}^{l}$ satisfying: (1) $S$ is a $l+1$-dimensional complex variety with normal crossings; (2) All fibers of $\tilde{\pi}$ are admissible. Denote by $j_{S}$ the 
complex structure on $S, J$ a $\omega$-tamed fiberwise almost complex structure on $\mathcal{X}_{Z}$. Let $\tilde{\pi}_{1}$ and $\tilde{\pi}_{2}$ be the projection maps from $S \times \mathcal{X}_{Z}$ to $S$ and $\mathcal{X}_{Z}$ respectively. An inhomogeneous term $\tilde{\nu}$ on $S \times \mathcal{X}_{Z}$ is defined to be a section of $\operatorname{Hom}\left(\tilde{\pi}_{1}^{*} T S, \tilde{\pi}_{2}^{*} E\right) \rightarrow S \times \mathcal{X}_{Z}$ satisfying

(i) $\tilde{\nu}$ is continuous and differentiable on each stratum;

(ii) Condition $2^{\prime}$. Using the notation of Remark 1.2, for any $z_{\infty} \in Z$,

$$
\left\|\left.\tilde{\nu}\right|_{S \times z_{r} \times V}-\left.\tilde{\nu}\right|_{S \times z_{\infty} \times V}\right\|_{C^{4}(S \times V)} \rightarrow 0, \text { as } z_{r} \rightarrow z_{\infty}, z_{r} \in U
$$

(iii) The anti- $J$-linear condition: $J \circ \tilde{\nu}=-\tilde{\nu} \circ j_{S}$.

The setting for the compactness theorem is: fix a degeneration of admissible curves $\tilde{\pi}: \mathcal{S} \rightarrow \Delta$, assume that $\tilde{\omega}$ is the induced fiberwise continuous symplectic form on $\mathcal{X}_{Z} \times \Delta \rightarrow Z \times \Delta$ with bounded $H^{k, p}$ norm on each stratum, $\tilde{J}$ is a $\omega$-tamed fiberwise almost complex structure, and $\tilde{\nu}_{1}, \cdots, \tilde{\nu}_{m}$ are families of fiberwise inhomogeneous terms on $S$. Let $\left\{t_{r}\right\}$ be a sequence in $\Delta$ converging to the origin as $r \rightarrow \infty$, set $\omega^{(r)}=\left.\tilde{\omega}\right|_{\mathcal{X}_{Z} \times t_{r}}, J^{(r)}=$ $\left.\tilde{J}\right|_{\mathcal{X}_{Z} \times t_{r}}, \Sigma^{(r)}=\tilde{\pi}^{-1}\left(t_{r}\right)$. Further assume that each $\Sigma^{(r)}$ consists of $m$ components $\left(\Sigma_{1}^{(r)}, \cdots, \Sigma_{m}^{(r)}\right)$, and for any fixed $i, \Sigma_{i}^{(r)}$ are diffeomorphic to each other for any $r$. Set $\nu^{(r)}=\left(\nu_{1}^{(r)}, \cdots, \nu_{m}^{(r)}\right)$, where $\nu_{i}^{(r)}=\left.\tilde{\nu}_{i}\right|_{\Sigma_{i}^{(r)} \times \mathcal{X}_{z}}, \nu^{(r)}$ is the inhomogeneous term on $\Sigma^{(r)}$. Note that $\Sigma_{i}^{(r)}$ converges to the $i$-th component $\Sigma_{i}^{(\infty)}$ of an admissible curve $\Sigma^{(\infty)}=\tilde{\pi}^{-1}(0)$, and $\nu_{i}^{(r)}$ converges to the $i$-th component $\nu_{i}^{(\infty)}=\left.\tilde{\nu}_{i}\right|_{\Sigma_{i}^{(\infty)} \times \mathcal{X}_{Z}}$ of inhomogeneous term $\nu^{(\infty)}=\left(\nu_{1}^{(\infty)}, \cdots, \nu_{m}^{(\infty)}\right)$ on $\Sigma^{(\infty)} \times \mathcal{X}_{Z}$ in $C^{4}$-topology in the following sense: there are continuous maps $\tau_{i}^{(r)}: \Sigma_{i}^{(r)} \rightarrow \Sigma_{i}^{(\infty)}$ and compact set $K_{i}^{(r)}$ in $\Sigma_{i}^{(\infty)}$ satisfying : (1) $K_{i}^{(r)} \subset K_{i}^{(r+1)}, \cup K_{i}^{(r)}=\Sigma_{i}^{(\infty)} \backslash$ \{double points\}; (2) $\tau_{i}^{(r)}$ restricts to a diffeomorphism from $\left(\tau_{i}^{(r)}\right)^{-1}\left(K_{i}^{(r)}\right)$ onto $K_{i}^{(r)}$; (3) For each index $i$, index $s$, and each stratum $Z_{\alpha} \subset Z$, both

$$
\left\|j_{\Sigma_{i}^{(\infty)}} \circ d \tau_{i}^{(r)}-d \tau_{i}^{(r)} \circ j_{\Sigma_{i}^{(r)}}\right\|_{C^{4}\left(\left(\tau_{i}^{(r)}\right)^{-1}\left(K_{i}^{(s)}\right)\right)}
$$

and

$$
\left\|\nu_{i}^{(\infty)} \circ d \tau_{i}^{(r)}-\nu_{i}^{(r)}\right\|_{C^{4}\left(\left(\tau_{i}^{(r)}\right)^{-1}\left(K_{i}^{(s)}\right) \times \mathcal{X}_{z_{\alpha}}\right)}
$$

converge to zero as $r \rightarrow \infty$.

Given $f^{(r)} \in \mathcal{M}_{\left(A_{1}, \cdots, A_{m}\right)}^{v}\left(\Sigma^{(r)}, \mathcal{X}_{Z}, J^{(r)}, \nu^{(r)}\right)$, we say that $f^{(r)}$ converges to $f^{(\infty)}: \Sigma^{(\infty)} \rightarrow \mathcal{X}_{Z}$ if $\left\|f_{i}^{(\infty)} \circ \tau_{i}^{(r)}-f_{i}^{(r)}\right\|_{C_{\text {loc }}^{3}} \rightarrow 0$ as $r \rightarrow 0$. Here we 
need the trivialization of Remark 1.2 to define $C_{\text {loc }}^{3}$-norm, and we also need a trivialization of $V$ to define $f_{i}^{(\infty)} \circ \tau_{i}^{(r)}-f_{i}^{(r)}$.

Proposition 1.1 (Compactness Theorem). Let $f^{(r)}$ be in

$$
\mathcal{M}_{\left(A_{1}, \cdots, A_{m}\right)}^{v}\left(\Sigma^{(r)}, \mathcal{X}_{Z}, J^{(r)}, \nu^{(r)}\right) \text {. }
$$

Then there is an admissible curve $\tilde{\Sigma}$, which is the union of the smooth resolution $\tilde{\Sigma}^{(\infty)}$ of $\Sigma^{(\infty)}$ and finitely many smooth rational curves such that a subsequence of $\left\{f^{(r)}\right\}_{1}^{\infty}$ converges to a $\left(J^{(\infty)}, \nu\right)$-perturbed holomorphic map $f$ on $\tilde{\Sigma}$, where the fiberwise inhomogeneous term $\nu$ coincide with $\nu^{(\infty)}$ on $\tilde{\Sigma}^{(\infty)}$ and vanishes on those rational curves, and moreover we have $\left[f_{*}(\tilde{\Sigma})\right]=A_{1}+\cdots+A_{m}$ and $f(\tilde{\Sigma}) \subset V_{z}$, for some $z \in Z$.

Proof. Without loss of generality we may assume that $\Sigma^{(r)}$ is smooth, since otherwise we can consider the $\left\{\Sigma_{i}^{(r)}\right\}_{1}^{\infty}$ family or its resolution for each fixed $i$. In the rest part of proof, we will use $A$ instead of $A_{1}, \cdots, A_{m}$. We also make a reduction to eliminate the fiberwise inhomogeneous term $\nu^{(r)}$. Let $\mathcal{W}=S \times \mathcal{X}_{Z} . \mathcal{W}$ has a fiber bundle structure $\mathcal{W} \stackrel{\tilde{p}}{\rightarrow} Z, \tilde{p}(s, x)=p(x)$, and we introduce a fiberwise almost complex structure $J_{\mathcal{W}}^{(r)}$ on $\mathcal{W}$ as follows. Given the vector bundle $E_{\mathcal{W}} \rightarrow \mathcal{W}$ whose fiber is $\left.E_{\mathcal{W}}\right|_{(s, x)}=T_{s} S \times E_{x}$, if $(u, v)$ is a vector in $\left.E_{\mathcal{W}}\right|_{(s, x)}$, define

$$
J_{\mathcal{W}}^{(r)}(u, v)=\left(j_{S}(u), J^{(r)}(v)+\nu^{(r)}\left(j_{S}(u)\right)\right) .
$$

It is easy to check that this is a fiberwise almost complex structure on $\mathcal{W}$ tamed for sufficiently small $\nu^{(r)}$ by the fiberwise symplectic form $\omega_{\mathcal{W}}$ which is the sum of the pull back of $\omega_{S}$ and $\omega$ to $\mathcal{W}$. Moreover if we take the graph of $f^{(r)}, F^{(r)}: \Sigma^{(r)} \rightarrow \mathcal{W}, F^{(r)}(x)=\left(x, f^{(r)}(x)\right)$ where $x \in \Sigma^{(r)}$, then $F^{(r)}$ is a $J_{\mathcal{W}}^{(r)}$-holomorphic map. So, it suffices to show that a subsequence of $\left\{F^{(r)}\right\}_{1}^{\infty}$ converges to a holomorphic map $F: \tilde{\Sigma} \rightarrow \mathcal{W}$ with $\left[F_{*}(\Sigma)\right]=\left[F_{*}^{(r)}\left(\Sigma^{(r)}\right)\right]$, where $\tilde{\Sigma}$ is given in Proposition 1.1. Thus we have reduced the perturbed case to the non-perturbed case $\nu^{(r)}=0$. From now on we assume that each $f^{(r)}$ is $J^{(r)}$-holomorphic. Now we fix a $J^{(\infty)}$-compatible metric $h$ on $E$, which satisfies

(i) $h$ is continuous, and smooth on each smooth stratum $\mathcal{X}_{Z}$;

(ii) Condition 3. Using the notation of Remark 1.2, for any $z_{\infty} \in U$,

$$
\left\|\left.h\right|_{z_{r} \times V}-\left.h\right|_{z_{\infty} \times V}\right\|_{C^{4}(V)} \rightarrow 0, \text { as } z_{r} \rightarrow z_{\infty}, z_{r} \in U .
$$


Because $J^{(r)}$ depends continuously on $t_{r}$, there is a positive constant $c$ such that $\omega^{(r)}\left(\xi, J^{(r)} \xi\right) \geq c \cdot h(\xi, \xi)$, for all $z$ and sufficiently large $r$, where $\xi \in E_{z}$.

Lemma 1.2. There is a uniformly constant $C_{A}$ which depends only on $A$ and $c$, such that for any $f^{(r)} \in \mathcal{M}_{A}^{v}\left(\Sigma^{(r)}, \mathcal{X}_{Z}, J^{(r)}, 0\right)$, we have

$$
\int_{\Sigma_{(r)}}\left|d f^{(r)}\right|_{\mu_{r}, h}^{2} d \mu_{r} \leq C_{A}
$$

where the volume form $d \mu_{r}$ is defined using any conformal metric $\mu_{r}$ on $\Sigma^{(r)}$.

Proof. Fixing $\Sigma=\Sigma^{(r)}, f=f^{(r)}, \omega=\omega^{(r)}, J=J^{(r)}, \mu=\mu_{r}, f(\Sigma) \subset V_{z}$, we then have

$$
\left.\int_{\Sigma} f^{*} \omega\right|_{V_{z}}=\left.\int_{f(\Sigma)} \omega\right|_{V_{z}}=\left.\int_{A} \omega\right|_{V_{z}}
$$

Since $\left.\omega\right|_{V_{z}}$ is a continuous function of $t^{(r)}$ and $z$, the above integral is bounded by some constant independent of $t^{(r)}$ and $z$.

At each point $x \in V_{z}$, choose a local unitary frame $e_{1}, \cdots, e_{n} \in T_{x}^{1,0} V_{z}$ with respect to a metric $h$, and denote by $\left\{e_{i}^{*}\right\}_{1}^{n}$ the dual basis of $\left\{e_{i}\right\}_{1}^{n}$. The symplectic form can now be written as

$$
\left.\omega\right|_{V_{z}}=\omega_{i j}^{(2,0)}\left(t_{r}, z\right) e_{i}^{*} \wedge e_{j}^{*}+\omega_{i j}^{(1,1)}\left(t_{r}, z\right) e_{i}^{*} \wedge \overline{e_{j}^{*}}+\omega_{i j}^{(0,2)}\left(t_{r}, z\right) \overline{e_{i}^{*}} \wedge \overline{e_{j}^{*}}
$$

Let $\frac{\partial}{\partial u_{1}}, \frac{\partial}{\partial u_{2}}$ be a local orthonormal basis of $(\Sigma, \mu)$, such that

$$
j\left(\frac{\partial}{\partial u_{1}}\right)=\frac{\partial}{\partial u_{2}}, \quad j\left(\frac{\partial}{\partial u_{2}}\right)=-\frac{\partial}{\partial u_{1}},
$$

then the Cauchy-Riemann equation

$$
(d f+J \circ d f \circ j)\left(\frac{\partial}{\partial u_{1}}\right)=0
$$

can be written locally as

$$
f_{1}^{i}=-\sqrt{-1} f_{2}^{i}, \quad f_{1}^{\bar{i}}=\sqrt{-1} f_{2}^{\bar{i}}
$$

where

$$
d f\left(\frac{\partial}{\partial u_{1}}\right)=f_{1}^{i} e_{i}+f_{1}^{\bar{i}} \bar{e}_{i} \quad \text { and } \quad d f\left(\frac{\partial}{\partial u_{2}}\right)=f_{2}^{i} e_{i}+f_{2}^{\bar{i}} \bar{e}_{i}
$$


From these equations we deduce that

$$
\begin{aligned}
\left.f^{*} \omega\right|_{V_{z}}= & \omega_{i j}^{(2,0)}\left(t_{r}, z\right) f^{*}\left(e_{i}^{*} \wedge e_{j}^{*}\right)+\omega_{i j}^{(1,1)}\left(t_{r}, z\right) f^{*}\left(e_{i}^{*} \wedge \bar{e}_{j}^{*}\right) \\
& +\omega_{i j}^{(0,2)}\left(t_{r}, z\right) f^{*}\left(\overline{e_{i}^{*}} \wedge \bar{e}_{j}^{*}\right) \\
= & -\sqrt{-1} \omega_{i j}^{1,1}\left(t_{r}, z\right)\left(f_{1}^{i} f_{1}^{\bar{j}}+f_{2}^{i} f_{2}^{\bar{j}}\right) d u_{1} \wedge d u_{2}
\end{aligned}
$$

The fact that $\left.J\right|_{V_{z}}$ is a $\left.\omega\right|_{V_{z}}$-tamed almost complex structure implies that (see the choice of metric $h$ above)

$$
\begin{aligned}
& \left.\omega\right|_{V_{z}}\left(d f\left(\frac{\partial}{\partial u_{1}}\right), J \circ d f\left(\frac{\partial}{\partial u_{1}}\right)\right)+\left.\omega\right|_{V_{z}}\left(d f\left(\frac{\partial}{\partial u_{2}}\right), J \circ d f\left(\frac{\partial}{\partial u_{2}}\right)\right) \\
& \geq c \cdot\left(\left|d f\left(\frac{\partial}{\partial u_{1}}\right)\right|_{h}^{2}+\left|d f\left(\frac{\partial}{\partial u_{2}}\right)\right|_{h}^{2}\right)=c|d f|_{h}^{2} .
\end{aligned}
$$

On the other hand, direct computation shows that

$$
\begin{aligned}
& \left.\omega\right|_{V_{z}}\left(d f\left(\frac{\partial}{\partial u_{1}}\right), J \circ d f\left(\frac{\partial}{\partial u_{1}}\right)\right)+\left.\omega\right|_{V_{z}}\left(d f\left(\frac{\partial}{\partial u_{2}}\right), J \circ d f\left(\frac{\partial}{\partial u_{2}}\right)\right) \\
& =-\sqrt{-1} \omega_{i j}^{1,1}\left(t_{r}, z\right)\left(f_{1}^{i} f_{1}^{\bar{j}}+f_{2}^{i} f_{2}^{j}\right) d u_{1} \wedge d u_{2},
\end{aligned}
$$

and so the lemma follows.

Lemma 1.3. There are $\epsilon_{0}>0$ and $C>0$ which depend only on $A, \tilde{J}$, and $\tilde{\omega}$, such that for any $J$-holomorphic map $f^{(r)} \in \mathcal{M}_{A}^{v}\left(\Sigma^{(r)}, \mathcal{X}_{Z}, J^{(r)}, 0\right), f^{(r)}$ : $\Sigma^{(r)} \rightarrow V_{z_{r}}$ for some $z_{r} \in Z$, and any conformal metric $\mu_{r}$ on $\Sigma^{(r)}$ with curvature bounded by 1 , if $\int_{B_{2 R}(x)}\left|d f^{(r)}\right|_{\mu_{r}}^{2} d \mu_{r} \leq \epsilon_{0}$ and the injectivity radius at $x$ is not less than $2 R$, where $x \in \Sigma^{(r)}, R>0$, we have

$$
\sup _{B_{R}(x)}\left|d f^{(r)}\right|_{\mu_{r}}^{2} \leq \frac{C}{R^{2}}
$$

where $B_{R}(x)$ is the geodesic ball centered at $x$ and with radius $R$. Consequently $\left\|f^{(r)}\right\|_{C^{4}\left(B_{R}(x)\right)} \leq C_{4}$ for some constant $C_{4}$ independent of $z_{r}$.

Proof. This is essentially Theorem 2.3 in [PW]. Here we briefly elaborate on the establishment of the Bochner type formula. Fix a connection on bundle $E$ which is compatible with the metric $h$ and the complex structure $J^{(\infty)}$. 
Suppose that in local trivialization in $V_{z}, f^{(r)}=\left(f_{1}^{(r)}, \cdots, f_{2 n}^{(r)}\right)$ and $B_{2 R}(x)$ has coordinates $u_{1}, u_{2}$. By direct computation, we have following formula

$$
\begin{aligned}
\Delta\left(d f^{(r)}\right)= & \nabla^{*} \nabla\left(d f^{(r)}\right) \\
= & \text { polynomials of } \frac{\partial f_{i}^{(r)}}{\partial u_{\alpha}} \text { up to degree } 3 \\
& + \text { linear combinations of } \frac{\partial^{2} f_{i}^{(r)}}{\partial u_{\alpha} \partial u_{\beta}} \cdot \frac{\partial f_{j}^{(r)}}{\partial u_{\gamma}}
\end{aligned}
$$

where the coefficients of the polynomial involve $\tilde{J}$, the metric $h$, the connection $\nabla$, and their derivatives in the direction of $V_{z_{r}}$.

Then

$$
\begin{aligned}
\Delta_{\mu_{r}}\left(\left|d f^{(r)}\right|_{\mu_{r}, h}^{2}\right)= & 2\left\langle\nabla^{*} \nabla\left(d f^{(r)}\right), d f^{(r)}\right\rangle-2\left\langle\nabla\left(d f^{(r)}\right), \nabla\left(d f^{(r)}\right)\right\rangle \\
= & \tilde{C}_{1}\left|d f^{(r)}\right|_{\mu_{r}, h}^{2}+\tilde{C}_{2}\left|d f^{(r)}\right|_{\mu_{r}, h}^{4}-\left\langle\nabla\left(d f^{(r)}\right), \nabla\left(d f^{(r)}\right)\right\rangle \\
& + \text { linear combinations of } \frac{\partial^{2} f_{i}^{(r)}}{\partial u_{\alpha} \partial u_{\beta}} \cdot \frac{\partial f_{j}^{(r)}}{\partial u_{\gamma}} \cdot \frac{\partial f_{k}^{(r)}}{\partial u_{\delta}} \\
\leq & C_{1}\left|d f^{(r)}\right|_{\mu_{r}, h}^{2}+C_{2}\left|d f^{(r)}\right|_{\mu_{r}, h}^{4} \\
& +\epsilon \cdot\left\langle\nabla\left(d f^{(r)}\right), \nabla\left(d f^{(r)}\right)\right\rangle \\
& +C(\epsilon)\left|d f^{(r)}\right|_{\mu_{r}, h}^{4}-\left\langle\nabla\left(d f^{(r)}\right), \nabla\left(d f^{(r)}\right)\right\rangle
\end{aligned}
$$

where we use the Cauchy-Schwartz inequality with $\epsilon \leq 1$ in the last step. Note that since $J^{(r)}, \omega^{(r)}, h, \nabla$, and their derivatives in the $V_{z_{r}}$ directions all depend continuously on the parameters $t_{r}$ and $z_{r}$, we can choose constants $C_{1}, C_{2}, C(\epsilon)$ to be independent of $t_{r}, z_{r}$. So we have

$$
\Delta_{\mu_{r}}\left(\left|d f^{(r)}\right|_{\mu_{r}, h}^{2}\right) \leq C_{1}\left|d f^{(r)}\right|_{\mu_{r}, h}^{2}+C_{2}\left|d f^{(r)}\right|_{\mu_{r}, h}^{4}
$$

and the lemma is proved.

Using the trivialization in Remark 1.2 to identify $V_{z_{r}}$ with $V_{z}$ and seeing that the image of $f^{(r)}$ lies in $V_{z}$, the rest of the proof of Proposition 1.1 becomes similar to the proof of Proposition 3.1 in [RT], and so we omit it. Observe that the image of the limit $f$ is contained in $V_{z}$. 
The above proposition tell us that the limit map in the compactification of the fiberwise moduli space are still those that could possibly appear in non-fiberwise case (the so-called Gromov-Uhlenbeck compactification). So if we could establish a transversality theorem in the fiberwise case, counting the dimension of boundary will go through as in non-fiberwise case. It is this transversality theorem we now turn to.

\subsection{Several Transversality Results.}

Definition 1.3. Let $\Sigma, J$, and $\nu$ be as in Definition 1.2. A fiberwise $\Sigma$ cusp $(J, \nu)$-perturbed map $f$ is a continuous map from $\Sigma^{\prime}$ to $V_{z}$ for some $z \in Z$, where the domain $\Sigma^{\prime}$ of $f$ is obtained by joining a chain of $S^{2}$ 's at some double points of $\Sigma$ to separate the two components at the double points, and then attaching some trees of $S^{2}$ 's. The restriction of $f$ to $\Sigma$ is a $(J, \nu)$-perturbed holomorphic map and its restriction to the $S^{2}$ 's is $J$ holomorphic. We call components of $\Sigma$ principal components and the others bubble components. A fiberwise $\Sigma$-cusp curve is an equivalence class of cusp maps modulo the parameterization groups of bubbles.

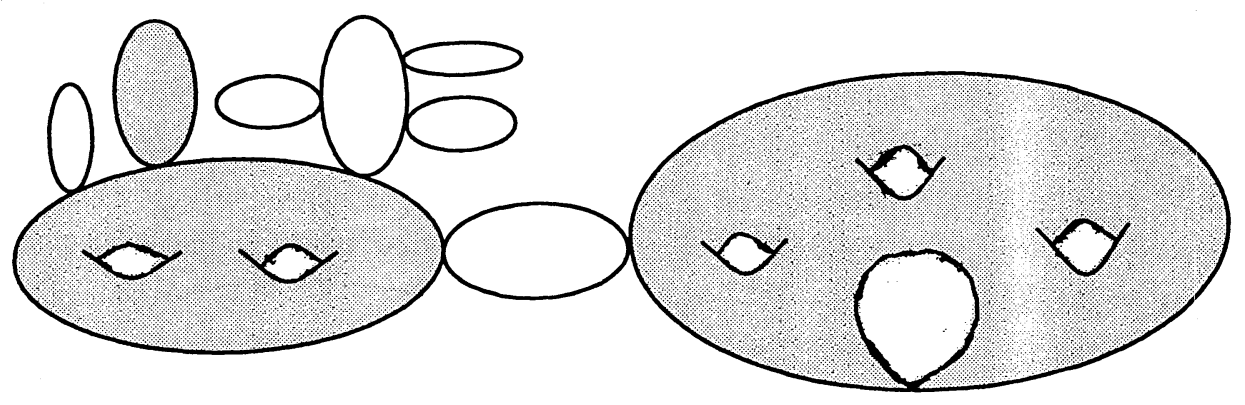

Figure 1: Domain of a cusp curve

By Proposition 1.1, we can compactify $\mathcal{M}_{\left(A_{1}, \cdots, A_{m}\right)}^{v}\left(\Sigma, \mathcal{X}_{Z}, J, \nu\right)$ by adding all possible fiberwise $\Sigma$-cusp curves with total homology class $A=$ $A_{1}+\cdots+A_{m}$. We divide the set of fiberwise cusp curves by some equivalence relations: 
(i) Some of the bubble components may be multiple covering maps, in this case we simply forget the multiplicity and take the reduced map onto its image;

(ii) Adjacent or consecutive bubbles have the same image, and we collapse them into one bubble. Note that the resulting curves are still fiberwise cusp curves with possibly different total homology class.

Let $\overline{\mathcal{M}}_{\left(A_{1}, \cdots, A_{m}\right)}^{v}\left(\Sigma, \mathcal{X}_{Z}, J, \nu\right)$ be the quotient of the fiberwise cusp curve compactification of $\mathcal{M}_{\left(A_{1}, \cdots, A_{m}\right)}^{v}\left(\Sigma, \mathcal{X}_{Z}, J, \nu\right)$ by above equivalence relation. Fix $k \geq 4, q>p \geq 2, l \geq k+1$. Let $\mathcal{P}_{k-1, p}\left(\mathcal{X}_{Z}, \omega\right) \rightarrow \mathcal{J}_{l, q}^{v}\left(\mathcal{X}_{Z}, \omega\right)$ be a vector bundle, the fiber over $J \in \mathcal{J}_{l, q}^{v}\left(\mathcal{X}_{Z}, \omega\right)$ is the space of all inhomogeneous term defined by $J . \mathcal{P}_{k-1, p}\left(\mathcal{X}_{Z}, \omega\right)$ is a smooth Banach manifold.

Proposition 1.2. Under Assumption ( $\star$ ), given any compact finite dimensional pseudo-submanifold $Z$ of $Y$, for a generic $(J, \nu) \in \mathcal{P}_{k-1, p}\left(\mathcal{X}_{Z}, \omega\right)$,

$$
\mathcal{M}_{\left(A_{1}, \cdots, A_{m}\right)}^{v}\left(\Sigma, \mathcal{X}_{Z}, J, \nu\right)
$$

is an oriented pseudo-manifold of real dimension $2 C_{1}(V) \cdot A+2 n(1-g(\Sigma))+$ $\operatorname{dim} Z$, and $\overline{\mathcal{M}}_{\left(A_{1}, \cdots, A_{m}\right)}^{v}\left(\Sigma, \mathcal{X}_{Z}, J, \nu\right) \backslash \mathcal{M}_{\left(A_{1}, \cdots, A_{m}\right)}^{v}\left(\Sigma, \mathcal{X}_{Z}, J, \nu\right)$ consists of finite many pieces (called strata) and each stratum is branchedly covered by a smooth manifold of codimension at least 2. Here $A=A_{1}+\cdots+A_{m}$ and $g(\Sigma)$ is the genus of the stable curve. More precisely, we have

$$
\mathcal{M}_{\left(A_{1}, \cdots, A_{m}\right)}^{v}\left(\Sigma, \mathcal{X}_{Z}, J, \nu\right)
$$

consists of smooth stratum $\mathcal{M}_{\left(A_{1}, \cdots, A_{m}\right)}^{v}\left(\Sigma, \mathcal{X}_{Z^{\alpha}}, J, \nu\right)$ of dimension $2 C_{1}(V) \cdot A+2 n(1-g(\Sigma))+\operatorname{dim} Z^{\alpha}$ where $Z^{\alpha}$ run through all smooth stratum of $Z$;

(ii)

$$
\overline{\mathcal{M}}_{\left(A_{1}, \cdots, A_{m}\right)}^{v}\left(\Sigma, \mathcal{X}_{Z}, J, \nu\right) \backslash \mathcal{M}_{\left(A_{1}, \cdots, A_{m}\right)}^{v}\left(\Sigma, \mathcal{X}_{Z}, J, \nu\right)
$$

consists of all

$$
\overline{\mathcal{M}}_{\left(A_{1}, \cdots, A_{m}\right)}^{v, p}\left(\Sigma, \mathcal{X}_{Z^{\alpha}}, J, \nu\right) \backslash \mathcal{M}_{\left(A_{1}, \cdots, A_{m}\right)}^{v}\left(\Sigma, \mathcal{X}_{Z^{\alpha}}, J, \nu\right)
$$

(iii) Each $\overline{\mathcal{M}}_{\left(A_{1}, \cdots, A_{m}\right)}^{v, p}\left(\Sigma, \mathcal{X}_{Z^{\alpha}}, J, \nu\right) \backslash \mathcal{M}_{\left(A_{1}, \cdots, A_{m}\right)}^{v}\left(\Sigma, \mathcal{X}_{Z^{\alpha}}, J, \nu\right)$ consists of finite many pieces, each of which is branched covered by a smooth manifold of codimension at least 2 in $\mathcal{M}_{\left(A_{1}, \cdots, A_{m}\right)}^{v}\left(\Sigma, \mathcal{X}_{Z^{\alpha}}, J, \nu\right)$, 
where $\overline{\mathcal{M}}_{\left(A_{1}, \cdots, A_{m}\right)}^{v, p}\left(\Sigma, \mathcal{X}_{Z^{\alpha}}, J, \nu\right)$ stands for the partial compactification of $\mathcal{M}_{\left(A_{1}, \cdots, A_{m}\right)}^{v}\left(\Sigma, \mathcal{X}_{Z^{\alpha}}, J, \nu\right)$. By partial compactification, we mean that we do not include limits of family of curves $f^{(r)}$ in $\mathcal{M}_{A_{1}, \cdots, A_{m}}^{v}\left(\Sigma, \mathcal{X}_{Z^{\alpha}}, J, \nu\right)$ with image in $V_{z_{r}}$, where the limit point $z_{\infty}$ is in not in $Z^{\alpha}$.

Proof. By Proposition 1.1, all we need to show is: for a generic $(J, \nu)$, for each stratum $Z^{\alpha}$ of $Z$,

$$
\mathcal{M}_{\left(A_{1}, \cdots, A_{m}\right)}^{v}\left(\Sigma, \mathcal{X}_{Z^{\alpha}}, J, \nu\right)
$$

is a smooth manifold of dimension

$$
2 C_{1}(V) \cdot A+2 n(1-g(\Sigma))+\operatorname{dim} Z^{\alpha},
$$

and

$$
\overline{\mathcal{M}}_{\left(A_{1}, \cdots, A_{m}\right)}^{v, p}\left(\Sigma, \mathcal{X}_{Z^{\alpha}}, J, \nu\right) \backslash \mathcal{M}_{\left(A_{1}, \cdots, A_{m}\right)}^{v}\left(\Sigma, \mathcal{X}_{Z^{\alpha}}, J, \nu\right)
$$

consists of finite many pieces, each piece branch-covered by a smooth manifold of codimension at least 2 in $\mathcal{M}_{\left(A_{1}, \cdots, A_{m}\right)}^{v}\left(\Sigma, \mathcal{X}_{Z^{\alpha}}, J, \nu\right)$. We divide the proof into three steps.

Step 1. The stratum decomposition. A stratum in

$$
\overline{\mathcal{M}}_{\left(A_{1}, \cdots, A_{m}\right)}^{v, p}\left(\Sigma, \mathcal{X}_{Z^{\alpha}}, J, \nu\right) \backslash \mathcal{M}_{\left(A_{1}, \cdots, A_{m}\right)}^{v}\left(\Sigma, \mathcal{X}_{Z^{\alpha}}, J, \nu\right)
$$

is a set of fiberwise cusp curves satisfying : (1) They have domain of the same homeomorphic type; (2) Each connected component has a fixed homology class; (3) The components which have the same image are the same for each curve even though these components may not be adjacent to each other. We denote by $D$ a set of triples, each triple consists of these three datas: a homeomorphic type of the domain of the fiberwise cusp curves with intersection points, a homology class associated to each component, and a specification of components with the same image.

Definition 1.4. Let $D$ be given as above, $\Sigma_{1}, \cdots, \Sigma_{m}$ be the principal components of $\Sigma$, and $B_{1}, \cdots, B_{k}$ be the bubble components of $D$. $D$ is called $A$-admissible if there are positive integers $b_{1}, \cdots, b_{k}$ such that $A=\sum_{1}^{m}\left[P_{i}\right]+\sum_{1}^{m} b_{j}\left[B_{j}\right]$, where $\left[P_{1}\right], \cdots,\left[P_{m}\right],\left[B_{1}\right], \cdots,\left[B_{k}\right]$ denote the homology classes associated with $\Sigma_{1}, \cdots, \Sigma_{m}, B_{1}, \cdots, B_{k}$. We say that $D$ is $(J, \nu)$-effective if every principal component can be represented by a $(J, \nu)$ map and every bubble component can be represented by a J-holomorphic map. 
Denote by $\mathcal{D}_{A, \Sigma}^{J, \nu}$ the set of all $A$-admissible, $(J, \nu)$-effective $D$ 's. It is proved in [RT] that $\mathcal{D}_{A, \Sigma}^{J, \nu}$ is a finite set. For each $D \in \mathcal{D}_{A, \Sigma}^{J, \nu}$, let $\mathcal{M}_{D}^{v}\left(\Sigma, \mathcal{X}_{Z^{\alpha}}, J, \nu\right)$ be the stratum in

$$
\overline{\mathcal{M}}_{\left(A_{1}, \cdots, A_{m}\right)}^{v, p}\left(\Sigma, \mathcal{X}_{Z^{\alpha}}, J, \nu\right) \backslash \mathcal{M}_{\left(A_{1}, \cdots, A_{m}\right)}^{v}\left(\Sigma, \mathcal{X}_{Z^{\alpha}}, J, \nu\right)
$$

specified by $D$.We perform one more reduction by identifying the domains of those components which have the same image, and change the homology class accordingly. Suppose that the resulting new domain and homology class of each component are specified by $\bar{D}$. Denote by $\mathcal{M}_{\bar{D}}^{v}\left(\Sigma, \mathcal{X}_{Z^{\alpha}}, J, \nu\right)$ the space of cusp $(J, \nu)$-maps whose domain and the homology class of each components are specified by $\bar{D}$. Then for each $f \in \mathcal{M}_{\bar{D}}^{v}\left(\Sigma, \mathcal{X}_{Z^{\alpha}}, J, \nu\right)$, different bubble components have different image and each bubble map is somewhere injective. However the image of a principal component may be only one point. We identify $\mathcal{M}_{D}^{v}\left(\Sigma, \mathcal{X}_{Z^{\alpha}}, J, \nu\right)$ with $\mathcal{M}_{\bar{D}}^{v}\left(\Sigma, \mathcal{X}_{Z^{\alpha}}, J, \nu\right)$ below.
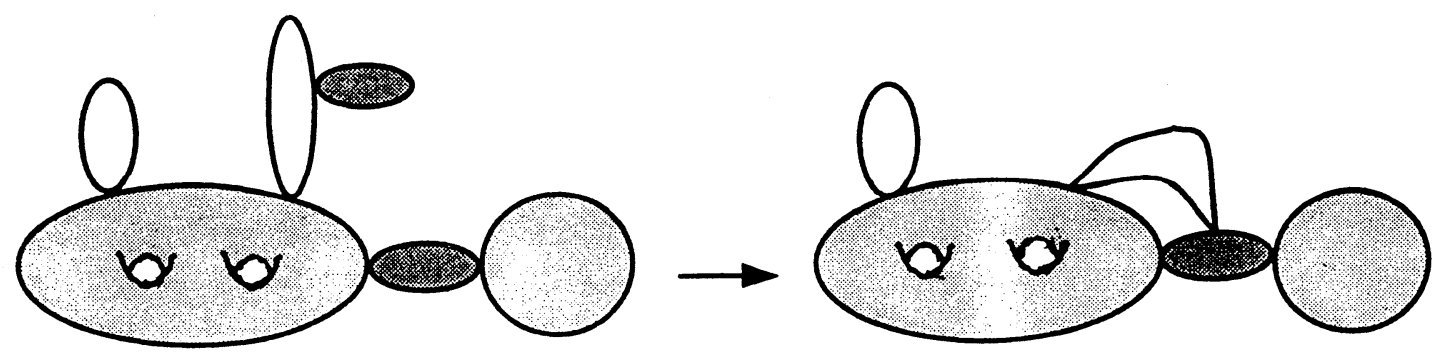

Figure 2: Creating a cycle in the domain

Again let $\Sigma_{1}, \cdots, \Sigma_{m}$ be the principal components, $\tilde{\Sigma}_{1}, \cdots, \tilde{\Sigma}_{m}$ be their smooth resolution $\left(\Sigma_{i}\right.$ may have double points), and $B_{1}, \cdots, B_{k}$ be the bubble components. Now we begin to construct a smooth branched covering of $\mathcal{M}_{\bar{D}}^{v}\left(\Sigma, \mathcal{X}_{Z^{\alpha}}, J, \nu\right)$. Let $\Sigma_{\bar{D}}$ be the domain of the maps in $\mathcal{M}_{\bar{D}}^{v}\left(\Sigma, \mathcal{X}_{Z^{\alpha}}, J, \nu\right)$, 
and consider

$$
\begin{aligned}
& \widetilde{\mathcal{M}}_{\bar{D}}^{v}\left(\Sigma, \mathcal{X}_{Z^{\alpha}}, J, \nu\right)= \\
& \left\{f: \Sigma_{\bar{D}} \rightarrow \mathcal{X}_{Z^{\alpha}} \mid f\right. \text { is a continuous fiberwise map, } \\
& \left.\quad f\right|_{\Sigma_{i}} \in \mathcal{M}_{\left[P_{i}\right]}^{v}\left(\Sigma_{i}, \mathcal{X}_{Z^{\alpha}}, J, \nu_{i}\right),\left.f\right|_{B_{i}} \in \widetilde{\mathcal{M}}_{\left[B_{j}\right]}^{v, *}\left(S^{2}, \mathcal{X}_{Z^{\alpha}}, J, 0\right), \\
& \left.\quad \operatorname{Im}\left(f_{B_{j}}\right) \neq \operatorname{Im}\left(f_{B_{j^{\prime}}}\right), \text { if } j \neq j^{\prime}\right\},
\end{aligned}
$$

where $\widetilde{\mathcal{M}}_{\left[B_{j}\right]}^{v, *}\left(S^{2}, \mathcal{X}_{Z^{\alpha}}, J, 0\right) \subset \mathcal{M}_{\left[B_{j}\right]}^{v}\left(S^{2}, \mathcal{X}_{Z^{\alpha}}, J, 0\right)$ is the subspace of nonmultiple covering maps. The action of the reparametrization group on each bubble component induces a $G^{k}$ action on $\widetilde{\mathcal{M}}_{\bar{D}}^{v}\left(\Sigma, \mathcal{X}_{Z^{\alpha}}, J, \nu\right), G=P S L_{2}$, so $\mathcal{M}_{\bar{D}}^{v}\left(\Sigma, \mathcal{X}_{Z^{\alpha}}, J, \nu\right)=\widetilde{\mathcal{M}}_{\bar{D}}^{v}\left(\Sigma, \mathcal{X}_{Z^{\alpha}}, J, \nu\right) / G^{k}$. We first construct a covering of $\widetilde{\mathcal{M}}_{\bar{D}}^{v}\left(\Sigma, \mathcal{X}_{Z^{\alpha}}, J, \nu\right)$. Let $x_{1}^{i}, \cdots, x_{p_{i}}^{i} \in \Sigma_{i}$ be $p_{i}$ double points or marked points which are bubbling points. Let $h_{i}$ be the number of intersection points on $\Sigma_{i}$ and $h^{j}$ be the number of intersection points on the bubble component $B_{j}$. Consider the evaluation map

$$
\begin{aligned}
e_{\bar{D}}: & \prod_{i}\left(\mathcal{M}_{\left[P_{i}\right]}^{v}\left(\tilde{\Sigma}_{i}, \mathcal{X}_{Z^{\alpha}}, J, \nu_{i}\right) \times\left(\tilde{\Sigma}_{i}\right)^{h_{i}-p_{i}}\right) \times \\
& \prod_{j}\left(\widetilde{\mathcal{M}}_{\left[B_{j}\right]}^{v, *}\left(S^{2}, \mathcal{X}_{Z^{\alpha}}, J, 0\right) \times\left(S^{2}\right)^{h^{j}}\right) \longrightarrow \\
& \prod_{i} \mathcal{X}_{Z^{\alpha}}^{h_{i}} \times \prod_{j} \mathcal{X}_{Z^{\alpha}}^{h^{j}}
\end{aligned}
$$

where $e_{\bar{D}}$ is defined as follows:

For each $\tilde{\Sigma}_{i}$, we define

$$
\begin{aligned}
& e_{P_{i}}: \mathcal{M}_{\left[P_{i}\right]}^{v}\left(\tilde{\Sigma}_{i}, \mathcal{X}_{Z^{\alpha}}, J, \nu_{i}\right) \times\left(\tilde{\Sigma}_{i}\right)^{h_{i}-p_{i}} \longrightarrow \mathcal{X}_{Z^{\alpha}}^{h_{i}} \\
& e_{P_{i}}\left(f, \tilde{x}_{1}, \cdots, \tilde{x}_{h_{i}-p_{i}}\right)=\left(f\left(x_{1}^{i}\right), \cdots, f\left(x_{p_{i}}^{i}\right), f\left(\tilde{x}_{1}\right), \cdots, f\left(\tilde{x}_{h_{i}-p_{i}}\right)\right) .
\end{aligned}
$$

For each $B_{j}$, we define

$$
\begin{aligned}
& e_{B_{j}}: \widetilde{\mathcal{M}}_{\left[B_{j}\right]}^{v, *}\left(S^{2}, \mathcal{X}_{Z^{\alpha}}, J, 0\right) \times\left(S^{2}\right)^{h^{j}} \longrightarrow \mathcal{X}_{Z^{\alpha}}^{h^{j}} \\
& e_{B_{j}}\left(f, y_{1}, \cdots, y_{h^{j}}\right)=\left(f\left(y_{1}\right), \cdots, f\left(y_{h^{j}}\right)\right) .
\end{aligned}
$$

Then $e_{\bar{D}}=\prod_{i} e_{P_{i}} \times \prod_{j} e_{B_{j}}$. Recall that if $M, N$ are submanifolds of a manifold $X$, then $M \cap N$ can be interpreted as $(M \times N) \cap \Delta$, where $\Delta$ is the diagonal of $X \times X$. So we can realize the intersection pattern specified by 
$\bar{D}$ by constructing a "diagonal" in the product. Let $z_{1}, \cdots, z_{t_{\bar{D}}}$ be all the intersection points. For each $z_{s}$, let

$$
I_{s}=\left\{\tilde{\Sigma}_{i_{1}}, \cdots, \tilde{\Sigma}_{i_{q}}, B_{j_{1}}, \cdots, B_{j_{r}}\right\}
$$

be the set of components which intersect at $z_{s}$. Now we construct a fiberwise product $\mathcal{X}_{Z^{\alpha}, s}^{v}$ of $\mathcal{X}_{Z^{\alpha}}$ such that its diagonal describes the intersection at $z_{s}$. This is done as follows: We allocate one or two factors from each of $\mathcal{X}_{Z^{\alpha}}^{h_{i_{1}}}, \cdots, \mathcal{X}_{Z^{\alpha}}^{h_{i_{q}}}$, according to whether or not $z_{s}$ is a self-intersection point of the corresponding principal component. We also allocate one factor from each of the $\mathcal{X}_{Z{ }^{\alpha}}^{h_{k}}, k=1, \cdots, r$. Here $\mathcal{X}_{Z}^{h_{i}}$ and $\mathcal{X}_{Z}^{h^{j}}$ are the images of $e_{P_{i}}$ and $e_{B_{j}}$, respectively. Then we take the fiberwise product of these factors over $Z^{\alpha}$, denote it by $\mathcal{X}_{Z^{\alpha}, s}^{v}$, and denote its diagonal by $\Delta_{Z^{\alpha}, s}^{v}$. Then

$$
\Delta_{Z^{\alpha}, \bar{D}}^{v}=\Delta_{Z^{\alpha}, 1}^{v} \times \cdots \times \Delta_{Z^{\alpha}, t_{\bar{D}}}^{v} \subset \mathcal{X}_{Z^{\alpha}}^{\sum h_{i}+\sum h^{j}}
$$

is the "diagonal" realizing the intersection pattern between components of $\bar{D}$. Let $\pi$ be the natural projection from

$$
\begin{array}{r}
\prod_{i}\left(\mathcal{M}_{\left[P_{i}\right]}^{v}\left(\tilde{\Sigma}_{i}, \mathcal{X}_{Z^{\alpha}}, J, \nu_{i}\right) \times\left(\tilde{\Sigma}_{i}\right)^{h_{i}-p_{i}}\right) \\
\quad \times \prod_{j}\left(\widetilde{\mathcal{M}}_{\left[B_{j}\right]}^{v, *}\left(S^{2}, \mathcal{X}_{Z^{\alpha}}, J, 0\right) \times\left(S^{2}\right)^{h^{j}}\right)
\end{array}
$$

onto

$$
\prod_{i} \mathcal{M}_{\left[P_{i}\right]}^{v}\left(\tilde{\Sigma}_{i}, \mathcal{X}_{Z^{\alpha}}, J, \nu_{i}\right) \times \prod_{j} \widetilde{\mathcal{M}}_{\left[B_{j}\right]}^{v, *}\left(S^{2}, \mathcal{X}_{Z^{\alpha}}, J, 0\right) .
$$

Then $\widetilde{\mathcal{M}}_{\bar{D}}^{v}\left(\Sigma, \mathcal{X}_{Z^{\alpha}}, J, \nu\right) \subset \pi\left(e_{\bar{D}}^{-1}\left(\Delta_{Z^{\alpha}, \bar{D}}^{v}\right)\right)$. But they might not be equal because we require that bubble components have different images. Define $\tilde{\mathcal{N}}_{\bar{D}}^{v}\left(\Sigma, \mathcal{X}_{Z^{\alpha}}, J, \nu\right)$ to be

$$
e_{\bar{D}}^{-1}\left(\Delta_{Z^{\alpha}, \bar{D}}^{v}\right) \cap \pi^{-1}\left(\widetilde{\mathcal{M}}_{\bar{D}}^{v}\left(\Sigma, \mathcal{X}_{Z^{\alpha}}, J, \nu\right)\right),
$$

and $\mathcal{N}_{\bar{D}}^{v}\left(\Sigma, \mathcal{X}_{Z^{\alpha}}, J, \nu\right)$ to be

$$
\tilde{\mathcal{N}}_{\bar{D}}^{v}\left(\Sigma, \mathcal{X}_{Z^{\alpha}}, J, \nu\right) / G^{\Sigma h^{j}}
$$

Then

$$
\pi: \mathcal{N}_{\bar{D}}^{v}\left(\Sigma, \mathcal{X}_{Z^{\alpha}}, J, \nu\right) \rightarrow \mathcal{M}_{\bar{D}}^{v}\left(\Sigma, \mathcal{X}_{Z^{\alpha}}, J, \nu\right)
$$


is the required branched covering.

Step 2. We will show that for generic $(J, \nu)$, for each stratum $Z^{\alpha}$ in $Z$, $\mathcal{M}_{\left(A_{1}, \cdots, A_{m}\right)}^{v}\left(\Sigma, \mathcal{X}_{Z^{\alpha}}, J, \nu\right)$ is a smooth oriented manifold of real dimension $2 C_{1}(V) \cdot A+2 n(1-g(\Sigma))+\operatorname{dim} Z^{\alpha}$, and $\mathcal{N}_{\bar{D}}^{v}\left(\Sigma, \mathcal{X}_{Z^{\alpha}}, J, \nu\right)$ is a smooth manifold of real dimension

$$
\begin{aligned}
& \sum\left(2 C_{1}(V) \cdot\left[P_{i}\right]+2 n\left(1-g_{i}\right)\right)+\sum\left(2 C_{1}(V) \cdot\left[B_{j}\right]+2 n-6\right) \\
& \quad+2 h_{\bar{D}}-2 u_{\Sigma}-2 s_{D}-2 n\left(h_{\bar{D}}-t_{\bar{D}}\right)+\operatorname{dim} Z^{\alpha},
\end{aligned}
$$

where $g_{i}$ is the genus of $\Sigma_{i}, u_{\Sigma}$ is twice of the number of double points of $\Sigma, s_{D}$ is the number of marked points which are bubbling points, $t_{\bar{D}}$ is the number of intersection points of $\bar{D}$ and $h_{\bar{D}}=\sum h_{i}+\sum h^{j}$. By bubbling points we mean there is a bubble attaching to that point.

The basic idea of the proof is due to D. McDuff [M1]. Let $\mathcal{P}_{k-1, p}\left(\mathcal{X}_{Z}, \omega\right) \rightarrow \mathcal{J}_{l, q}^{v}\left(\mathcal{X}_{Z}, \omega\right)$ be the vector bundle defined at the beginning of this subsection. Define

$$
\begin{aligned}
\operatorname{Map}_{k, p}^{v}\left(\tilde{\Sigma}_{i}, \mathcal{X}_{Z^{\alpha}},\left[P_{i}\right]\right) & =\left\{f_{i}: \tilde{\Sigma}_{i} \rightarrow \mathcal{X}_{Z^{\alpha}} \mid f_{i}\right. \text { is a fiber map } \\
& \text { with bounded } \left.H^{k, p} \text { norm, }\left[f_{i}\left(\tilde{\Sigma}_{i}\right)\right]=\left[P_{i}\right]\right\}, \\
\operatorname{Map}_{k, p}^{v}\left(S^{2}, \mathcal{X}_{Z^{\alpha}},\left[B_{j}\right]\right)= & \left\{f^{j}: S^{2} \rightarrow \mathcal{X}_{Z^{\alpha}} \mid f^{j}\right. \text { is a fiber map } \\
& \text { with bounded } \left.H^{k, p} \text { norm, }\left[f^{j}\left(S^{2}\right)\right]=\left[B_{j}\right]\right\} .
\end{aligned}
$$

(Here we require all the maps from $S^{2}$ to $\mathcal{X}_{Z^{\alpha}}$ to be somewhere injective). Let

$$
\Gamma_{k-1, p}\left(T \tilde{\Sigma}_{i}, e_{P_{i}}^{*}\left(\left.E\right|_{\mathcal{X}_{Z^{\alpha}}}\right)\right) \text { and } \Gamma_{k-1, p}\left(T S^{2}, e_{B_{j}}^{*}\left(\left.E\right|_{\mathcal{X}_{Z^{\alpha}}}\right)\right)
$$

be vector bundles over

$$
\prod_{i} \operatorname{Map}_{k, p}^{v}\left(\tilde{\Sigma}_{i}, \mathcal{X}_{Z^{\alpha}},\left[P_{i}\right]\right) \times \prod_{j} M a p_{k, p}^{v}\left(S^{2}, \mathcal{X}_{Z^{\alpha}},\left[B_{j}\right]\right) \times \mathcal{P}_{k-1, p}\left(\mathcal{X}_{Z}, \omega\right)
$$

whose fibers over a point $\left(f_{i}, f^{j}, J, \nu\right)$ are sections of $\Omega_{\Sigma_{i}}^{0,1}\left(f_{i}^{*}(E)\right)$ and $\Omega_{S^{2}}^{0,1}\left(f^{j *}(E)\right)$, respectively, with bounded $H^{k-1, p}$ norm. Then we obtain an infinite dimensional bundle with a section $S$ which is a partial differential 
operator,

$$
\begin{aligned}
& \prod_{i} \Gamma_{k-1, p}\left(T \tilde{\Sigma}_{i}, e_{P_{i}}^{*}(E)\right) \times \prod_{j} \Gamma_{k-1, p}\left(T S^{2}, e_{B_{j}}^{*}(E)\right) \longrightarrow \\
& \prod_{i} M p_{k, p}^{v}\left(\tilde{\Sigma}_{i}, \mathcal{X}_{Z^{\alpha}},\left[P_{i}\right]\right) \times \prod_{j} M a p_{k, p}^{v}\left(S^{2}, \mathcal{X}_{Z^{\alpha}},\left[B_{j}\right]\right) \times \mathcal{P}_{k-1, p}\left(\mathcal{X}_{Z}, \omega\right), \\
& S\left(f_{i}, f^{j}, J, \nu\right)=\left(\bar{\partial}_{J} f_{i}-\left.\nu_{i}\right|_{\Sigma_{i}}, \bar{\partial}_{J} f^{j}\right) .
\end{aligned}
$$

Lemma 1.4. The zero section of the above bundle is regular for the section S. i.e.,

$$
\widetilde{\mathcal{M}}_{\left(\left[P_{i}\right],\left[B_{j}\right], \mathcal{P}\right)}^{v}\left(\mathcal{X}_{Z^{\alpha}}\right)=\left\{\left(f_{i}, f^{j}, J, \nu\right) \mid S\left(f_{i}, f^{j}, J, \nu\right)=0\right\}
$$

is a Banach submanifold of

$$
\prod_{i} \operatorname{Map} p_{k, p}^{v}\left(\tilde{\Sigma}_{i}, \mathcal{X}_{Z^{\alpha}},\left[P_{i}\right]\right) \times \prod_{j} M a p_{k, p}^{v}\left(S^{2}, \mathcal{X}_{Z^{\alpha}},\left[B_{j}\right]\right) \times \mathcal{P}_{k-1, p}\left(\mathcal{X}_{Z}, \omega\right) .
$$

Proof. For any point $z \in Z^{\alpha}$, choose a neighborhood $U$ of $z$ in $Z^{\alpha}$, such that if we fix a trivialization $\left.\mathcal{X}_{Z^{\alpha}}\right|_{U}=p^{-1}(U) \cong U \times V$ and $T p^{-1}(U) \cong T U \times T V$, then the tangent space at $\left(f_{i}, f^{j}, J, \nu\right)$ is

$$
\begin{aligned}
& T_{f_{i}} \operatorname{Map}_{k, p}^{v}\left(\tilde{\Sigma}_{i}, \mathcal{X}_{Z^{\alpha}},\left[P_{i}\right]\right)=\Gamma_{k, p}\left(\tilde{\Sigma}_{i}, f_{i}^{*} T V_{z}\right) \times T_{z} Z^{\alpha}, \\
& T_{f^{j}} \operatorname{Map}_{k, p}^{v}\left(S^{2}, \mathcal{X}_{Z^{\alpha}},\left[B^{j}\right]\right)=\Gamma_{k, p}\left(S^{2}, f^{j *} T V_{z}\right) \times T_{z} Z^{\alpha}, \\
& T_{J} \mathcal{J}_{l, q}^{v}\left(\mathcal{X}_{Z}, \omega\right)=\Gamma_{l, q}(E n d(E, J)),
\end{aligned}
$$

where $\pi \circ f_{i}=z, \pi \circ f^{j}=z$ and $\operatorname{End}(E, J)$ is the set of all the endmorphism of $E$. Those endmorphism anti-commutes with $J$ and is self-adjoint with respect to the metric $\omega(\cdot, J \cdot)$. So

$$
\begin{aligned}
& T_{(J, \nu)} \mathcal{P}_{k-1, p}\left(\mathcal{X}_{Z}, \omega\right)=\Gamma_{l, q}(E n d(E, J)) \times \prod_{i} \Gamma_{k-1, p}\left(\pi_{1}^{*} T \tilde{\Sigma}_{i}, \pi_{2}^{*} E\right) \\
& T_{\left(f_{i}, 0\right)} \Gamma_{k-1, p}\left(T \tilde{\Sigma}_{i}, e_{P_{i}}^{*}(E)\right) \\
& \quad=\Gamma_{k, p}\left(\tilde{\Sigma}_{i}, f_{i}^{*} T V_{z}\right) \times T_{z} Z^{\alpha} \times \Omega_{\left(\tilde{\Sigma}_{i}, k-1, p\right)}^{0,1}\left(f_{i}^{*} T V_{z}\right) \\
& T_{\left(f^{j}, 0\right)} \Gamma_{k-1, p}\left(T S^{2}, e_{B_{j}}^{*}(E)\right) \\
& \quad=\Gamma_{k, p}\left(S^{2}, f^{j *} T V_{z}\right) \times T_{z} Z^{\alpha} \times \Omega_{\left(S^{2}, k-1, p\right)}^{0,1}\left(f^{j *} T V_{z}\right)
\end{aligned}
$$


Note: the fiber directions of the above two tangent spaces are

$$
\Omega_{\left(\Sigma_{i}, k-1, p\right)}^{0,1}\left(f_{i}^{*} T V_{z}\right) \quad \text { and } \quad \Omega_{\left(S^{2}, k-1, p\right)}^{0,1}\left(f^{j *} T V_{z}\right)
$$

respectively. Let us compute the projection of the linearization of $S$ to the fiber direction at $\left(f_{i}, f^{j}, J, \nu\right)$ satisfying $S\left(f_{i}, f^{j}, J, \nu\right)=0$.

$$
\begin{aligned}
& \prod_{i} \Gamma_{k, p}\left(\tilde{\Sigma}_{i}, f_{i}^{*} T V_{z}\right) \times \prod_{j} \Gamma_{k, p}\left(S^{2}, f^{j *} T V_{z}\right) \times T_{z} Z^{\alpha} \times \\
& \Gamma_{l, q}(E n d(E, J)) \times \prod_{i} \Gamma_{k-1, p}\left(\pi_{1}^{*} T \tilde{\Sigma}_{i}, \pi_{2}^{*} E\right) \stackrel{D S\left(f_{i}, f^{j}, J, \nu\right)}{\longrightarrow} \\
& \prod_{i} \Gamma_{k, p}\left(\tilde{\Sigma}_{i}, f_{i}^{*} T V_{z}\right) \times \prod_{j} \Gamma_{k, p}\left(S^{2}, f^{j *} T V_{z}\right) \times T_{z} Z^{\alpha} \times \\
& \prod_{i} \Omega_{\left(\tilde{\Sigma}_{i}, k-1, p\right)}^{0,1}\left(f_{i}^{*} T V_{z}\right) \times \prod_{j} \Omega_{\left(S^{2}, k-1, p\right)}^{0,1}\left(f^{j *} T V_{z}\right) \stackrel{P}{\longrightarrow} \\
& \prod_{i} \Omega_{\left(\tilde{\Sigma}_{i}, k-1, p\right)}^{0,1}\left(f_{i}^{*} T V_{z}\right) \times \prod_{j} \Omega_{\left(S^{2}, k-1, p\right)}^{0,1}\left(f^{j *} T V_{z}\right) .
\end{aligned}
$$

We fix a Hermitian metric $h$ on $E$ which is compatible with $J$, also fix a connection $\nabla$ on $E$ which is compatible with this metric and the fiberwise almost complex structure. Further suppose that for any $z \in Z,\left.\nabla\right|_{V_{z}}$ has torsion $\frac{1}{4} N_{\left.J\right|_{V_{z}}}$, where $N_{\left.J\right|_{V_{z}}}$ is the Nijenhuis torsion tensor of $\left(V_{z},\left.J\right|_{V_{z}}\right)$. The construction of such a connection is the same as the construction of such a connection in the non-fiberwise case. $\nabla$ defines a parallel translations $P_{i, t}$ and $P_{t}^{j}$ along path $\left\{f_{i t}\right\}_{i=1}^{m}$ and $\left\{f_{t}^{j}\right\}_{j=1}^{k}$, respectively, on the bundle $E$, 
which will be used to define $D S$. We now can write down $\operatorname{Pr} \circ D S$ :

$$
\begin{aligned}
& \operatorname{Pr} \circ D S\left(f_{i}, f^{j}, J, \nu\right)(\xi, \eta, \gamma, w, \delta) \\
& =\operatorname{Pr} \circ D S(\xi, 0,0,0,0)+\operatorname{Pr} \circ D S(0, \eta, 0,0,0) \\
& \quad \quad+\operatorname{Pr} \circ D S(0,0, \gamma, 0,0)+\operatorname{Pr} \circ D S(0,0,0, w, 0) \\
& \quad \quad+\operatorname{Pr} \circ D S(0,0,0,0, \delta) \\
& \operatorname{Pr} \circ D S(\xi, 0,0,0,0) \\
& =\left(\left(\nabla \xi_{i}+\left.J\right|_{V_{z}} \circ \nabla \xi_{i} \circ j_{\Sigma_{i}}+\frac{1}{4} N_{\left.J\right|_{V_{z}}}\left(\partial_{\left.J\right|_{V_{z}}}\left(f_{i}\right), \xi_{i}\right)\right)_{i=1}^{m}, 0\right), \\
& \operatorname{Pr} \circ D S(0, \eta, 0,0,0) \\
& =\left(0,\left(\nabla \eta^{j}+\left.J\right|_{V_{z}} \circ \nabla \eta^{j} \circ j_{S^{2}}+\frac{1}{4} N_{\left.J\right|_{V_{z}}}\left(\partial_{\left.J\right|_{V_{z}}}\left(f^{j}\right), \eta^{j}\right)\right)_{j=1}^{k}\right), \\
& \operatorname{Pr} \circ D S(0,0,0, w, 0) \\
& =\left(\left(\frac{1}{2} w\left(f_{i}\right) \circ d f_{i} \circ j_{\Sigma_{i}}\right)_{i=1}^{m},\left(\frac{1}{2} w\left(f^{j}\right) \circ d f^{j} \circ j_{S^{2}}\right)_{j=1}^{k}\right)
\end{aligned}
$$

where

$$
\xi=\left(\xi_{1}, \cdots, \xi_{m}\right), \eta=\left(\eta^{1}, \cdots, \eta^{k}\right) \quad \text { and } \quad \delta=\left(\delta_{1}, \cdots, \delta_{m}\right)
$$

The computation of these formulas is exactly the same as in the nonfiberwise case since we may choose the families of maps varying in the directions of $\xi$ or $\eta$ to lie in the same fiber $V_{z}$ (see [M1]).

To compute $\operatorname{Pr} \circ D S(0,0, \gamma, 0,0)$, we choose families of maps,

$$
f_{i, t}=f_{i}, f_{t}^{j}=f^{j}, J_{t}=J, z_{t}
$$

satisfying

$$
\left.\frac{d z_{t}}{d t}\right|_{t=0}=\gamma, \quad \text { and } \quad \nu_{t}=\nu .
$$

Here we suppose $\operatorname{Im}\left(f_{t}\right) \subset V_{z_{t}}$, so 


$$
\begin{aligned}
& \operatorname{Pr} \circ D S(0,0, \gamma, 0,0) \\
& =\left(\left(\frac{d}{d t} P_{i, t}\left(d f_{i, t}+\left.J\right|_{V_{z_{t}}} \circ d f_{i, t} \circ j_{\Sigma_{i}}-\left.\nu_{i}\right|_{\Sigma_{i}}\right)\right)_{i=1}^{m},\right. \\
& \left.\left(\frac{d}{d t} P_{t}^{j}\left(d f_{t}^{j}+\left.J\right|_{V_{z_{t}}} \circ d f_{t}^{j} \circ j_{S^{2}}\right)\right)_{j=1}^{k}\right) \\
& =\left(\left(\nabla_{\gamma}\left(d f_{i}+\left.J\right|_{V_{z_{t}}} \circ d f_{i} \circ j_{\Sigma_{i}}-\left.\nu_{i}\right|_{\Sigma_{i}}\right)\right)_{i=1}^{m},\right. \\
& \left.\left(\nabla_{\gamma}\left(d f^{j}+\left.J\right|_{V_{z_{t}}} \circ d f^{j} \circ j_{S^{2}}\right)\right)_{j=1}^{k}\right) \\
& =\left(\left(\frac{d}{d t}\left(d f_{i}+\left.J\right|_{\Sigma_{z_{t}}} \circ d f_{i} \circ j_{\Sigma_{i}}-\left.\nu_{i}\right|_{\Sigma_{i}}\right)\right.\right. \\
& \left.+\Gamma_{\star \star}^{\star}\left(d f_{i}+\left.J\right|_{z_{z_{t}}} \circ d f_{i} \circ j_{\Sigma_{i}}-\left.\nu_{i}\right|_{\Sigma_{i}}\right)\right)_{i=1}^{m} \text {, } \\
& \left.\left(\frac{d}{d t}\left(d f^{j}+\left.J\right|_{V_{z_{t}}} \circ d f^{j} \circ j_{S^{2}}\right)+\Gamma_{\star \star}^{\star}\left(d f^{j}+\left.J\right|_{V_{z_{t}}} \circ d f^{j} \circ j_{S^{2}}\right)\right)_{j=1}^{k}\right)
\end{aligned}
$$

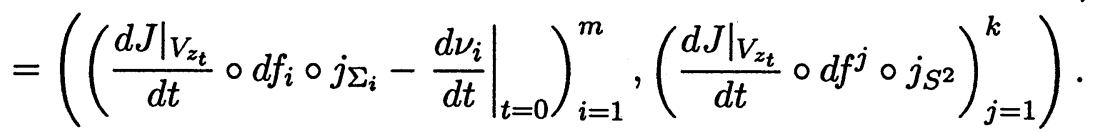

Note that in the last step we used $d f_{i}+\left.J\right|_{V_{z_{t}}} \circ d f_{i} \circ j_{\Sigma_{i}}-\nu_{i}=0, d f^{j}+$ $\left.J\right|_{V_{z_{t}}} \circ d f^{j} \circ j_{S^{2}}=0$ when $t=0$ and that $\Gamma_{\star \star}^{\star}$ denote the Christoffel symbol of the connection $\nabla$. Finally to compute $\operatorname{Pr} \circ D S(0,0,0,0, \delta)$, we choose families of maps $f_{i, t}=f_{i}, f_{t}^{j}=f^{j}, J_{t}=J, z_{t}=z$, and $\nu_{t}=\left(\nu_{i, t}\right)_{1}^{m}$ satisfying $\left.\frac{d \nu_{i, t}}{d t}\right|_{t=0}=\delta_{i}$, so

$$
\begin{aligned}
\operatorname{Pr} \circ D s\left(0,0,0,0, \delta_{i}\right) & \\
= & \left(\left(\frac{d}{d t} P_{i, t}\left(d f_{i}+\left.J\right|_{V_{z}} \circ d f_{i} \circ j_{\Sigma_{i}}-\nu_{i, t}\right)\right)_{i=1}^{m},\right. \\
& \left.\left(\frac{d}{d t} P_{t}^{j}\left(d f^{j}+\left.J\right|_{V_{y}} \circ d f^{j} \circ j_{S^{2}}\right)\right)_{j=1}^{k}\right) \\
= & \left(\delta_{i}, 0\right) .
\end{aligned}
$$

To show the section $S$ is transversal to the zero section, we need to show that $D S$ is surjective in the fiber direction, i.e. $\operatorname{Pr} \circ D S$ is a surjective map. According to Lemma 4.9 in [RT], $\operatorname{Pr} \circ D S$ is surjective for each fixed $z \in Z^{\alpha}$, where we specifically need the assumption that $f^{j}$ is somewhere injective. The only difference is that we need to show that the perturbation of $\left.J\right|_{V_{z}}$ 
and $\left.\nu\right|_{V_{z}}$ on $V_{z}$ used in the proof of Lemma 4.9 in [RT] can be extended to perturbations of $J$ and $\nu$ on $E$. This can clearly be done, so $\operatorname{Pr} \circ D S$ is surjective, and the lemma is proved.

To prove the proposition, all that is left is to show that

$$
e_{\bar{D}}: \widetilde{\mathcal{M}}_{\left(\left[P_{i}\right],\left[B_{j}\right], \mathcal{P}\right)}^{v}\left(\mathcal{X}_{Z^{\alpha}}\right) \times \prod_{i} \tilde{\Sigma}_{i}^{h_{i}-p_{i}} \times \prod_{j}\left(S^{2}\right)^{h^{j}} \longrightarrow \mathcal{X}_{Z^{\alpha}}^{\sum h_{i}+\Sigma h^{j}}
$$

is transversal to $\Delta_{Z^{\alpha}, \bar{D}}^{v}$. Let $\left(v_{1}, \cdots, v_{t_{\bar{D}}}\right)$ be a point in $\Delta_{Z^{\alpha}, \bar{D}}^{v}, I_{s}=$ $\left\{\tilde{\Sigma}_{i_{1}}, \cdots, \tilde{\Sigma}_{i_{p}}, B_{j_{1}}, \cdots, B_{j_{r}}\right\}$, and

$$
\left(f_{i}, f^{j}, J, \nu, x_{1}, \cdots, x_{\tilde{m}}, y_{1}, \cdots, y_{k}\right) \in e_{\bar{D}}^{-1}\left(v_{1}, \cdots, v_{t_{\bar{D}}}\right) .
$$

So $f_{i_{1}}\left(x_{i_{1}}\right)=\cdots=f_{i_{p}}\left(x_{i_{p}}\right)=f^{j_{1}}\left(y_{j_{1}}\right)=\cdots=f^{j_{r}}\left(y_{j_{r}}\right)=v_{s}$. By the Hartman-Winter Lemma [JS, Lemma 2.6.1], there are only finitely many accumulation points of $I m f^{j} \cap I m f^{j^{\prime}}$, since we require that $I m f^{j} \neq I m f^{j^{\prime}}$ for $j \neq j^{\prime}$. Therefore, for each $f^{j_{s}}$, we can choose a small disc $D_{j_{s}}$ around $y_{j_{s}}$ such that there is a smaller disc $D^{\prime} j_{s}$ satisfying that the annulus $D_{j_{s}}-D^{\prime} j_{s}$ does not intersect other bubble components. By the work of D. McDuff [M1], given any tangent vector $X \in T_{f^{j_{s}}\left(y_{j_{s}}\right)} V_{z}$, where $f(\Sigma) \subset V_{z}$, for some $z \in Z^{\alpha}$, there is a perturbation of almost complex structure $\left.J\right|_{V_{z}}$ to compatible almost complex structures $\left(\left.J\right|_{V_{z}}\right)_{t}$ on $V_{z}$ in a small neighborhood of $\left.I m f^{j_{s}}\right|_{D_{j_{s}}-D^{\prime} j_{s}}$, and also a perturbation $f_{t}^{j_{s}}$ of $f^{j_{s}}$ on $D_{j_{s}}$ such that $f_{t}^{j_{s}}$ is $\left(\left.J\right|_{V_{z}}\right)_{t}$ holomorphic, $f_{0}^{j_{s}}=f^{j_{s}}$, and $\left.\frac{d f_{t}^{j_{s}}}{d t}\left(y_{j_{s}}\right)\right|_{t=0}=X$. We can patch $f_{t}^{j_{s}}$ with $\left.f^{j_{s}}\right|_{S^{2}-D_{j_{s}}}$ to get $f_{t}^{j_{s}}$ defined on whole $S^{2}$ such that $\left.f_{t}^{j_{s}}\right|_{D_{j_{s}}-D^{\prime} j_{s}}$ does not intersect other bubble components either. Note that $\left(\left.J\right|_{V_{z}}\right)_{t}$ can be extended to a family of complex structure $J_{t}$ on $E$, i.e. we can choose perturbation $\left(\left.J\right|_{V_{z}}\right)_{t}$ which comes from $\mathcal{P}_{k-1, p}^{v}\left(\mathcal{X}_{Z}, \omega\right)$. Note that other bubbles are also $J_{t}$-holomorphic and $\left.\frac{d f_{t}^{j_{s}}}{d t}\left(y_{j_{s}}\right)\right|_{t=0}=X$.

For a principal component $f_{i_{s}}$, the argument is easier since we can perturb the inhomogeneous term. We can just choose an arbitrary perturbation $f_{i_{s}, t}$ on a small disc $D_{i_{s}}$ around $x_{i_{s}}$ such that $\left.\frac{d f_{i_{s}}, t}{d t}\left(x_{i_{s}}\right)\right|_{t=0}=X$, and then simply perturb $\left.\nu_{i}\right|_{V_{z}}$ to $\left(\left.\nu_{i}\right|_{V_{z}}\right)_{t}$ while keeping $\left(\left.\nu_{i_{s}}\right|_{V_{z}}\right)_{t}=\bar{\partial}_{J} f_{i_{s}, t}$ on the graph of $f_{i_{s}, t}$. Then $f_{i_{s}, t}$ satisfies an inhomogeneous Cauchy-Riemann equation with inhomogeneous term $\left(\nu_{i_{s}} \mid V_{z}\right)_{t}$. Note that $\left(\left.\nu_{i_{s}}\right|_{V_{z}}\right)_{t}$ can be extended to a family of elements in $\mathcal{P}_{k-1, p}^{v}\left(\mathcal{X}_{Z}, \omega\right)$. Applying this argument to each point $v_{s}$ and every point in $e_{\bar{D}}^{-1}\left(\Delta_{Z^{\alpha}, \bar{D}}^{v}\right)$, we have shown that the map $e_{\bar{D}}$ is transversal to $\Delta_{Z^{\alpha}, \bar{D}}^{v}$. Therefore $e_{\bar{D}}^{-1}\left(\Delta_{Z^{\alpha}, \bar{D}}^{v}\right)$ is a smooth Banach manifold. Moreover we have the following Fredholm map

$$
e_{\bar{D}}^{-1}\left(\Delta_{Z^{\alpha}, \bar{D}}^{v}\right) \stackrel{\tilde{\pi}}{\rightarrow} \mathcal{P}_{k-1, p}^{v}\left(\mathcal{X}_{Z}, \omega\right)
$$


By the Sard-Smale Theorem, for a generic element $(J, \nu)$ of $\mathcal{P}_{k-1, p}^{v}\left(\mathcal{X}_{Z}, \omega\right)$, its preimage $\tilde{\pi}^{-1}(J, \nu)$ is a smooth manifold (it is crucial here that $\mathcal{P}_{k-1, p}^{v}\left(\mathcal{X}_{Z}, \omega\right)$ has smooth structure). This implies that $\mathcal{M}_{\left(A_{1}, \cdots, A_{m}\right)}^{v}\left(\Sigma, \mathcal{X}_{Z^{\alpha}}, J, \nu\right)$ is a smooth manifold when we choose $\bar{D}$ with no bubbles $f^{j}$, and in general $\tilde{\mathcal{N}}_{\bar{D}}^{v}\left(\Sigma, \mathcal{X}_{Z^{\alpha}}, J, \nu\right)$ is smooth manifold. Since $G^{\sum h^{j}}$ acts freely on $\tilde{\mathcal{N}}_{\bar{D}}^{v}\left(\Sigma, \mathcal{X}_{Z^{\alpha}}, J, \nu\right), \mathcal{N}_{\bar{D}}^{v}\left(\Sigma, \mathcal{X}_{Z^{\alpha}}, J, \nu\right)=\tilde{\mathcal{N}}_{\bar{D}}^{v}\left(\Sigma, \mathcal{X}_{Z^{\alpha}}, J, \nu\right) / G^{\Sigma h^{j}}$ is smooth. Note that $G^{\sum h^{j}}$ acts freely on $e_{\bar{D}}^{-1}\left(\Delta_{Z^{\alpha}, \bar{D}}^{v}\right)$, so we can define a smooth manifold $E_{\bar{D}}^{-1}\left(\Delta_{Z^{\alpha}, \bar{D}}^{v}\right)=e_{\bar{D}}^{-1}\left(\Delta_{Z^{\alpha}, \bar{D}}^{v}\right) / G^{\sum h^{j}}$, a space that will be used below.

Step 3. A routine counting dimension argument gives the dimension formula (see $[\mathrm{RT}]$ ). We can see that the codimension of the boundary of the moduli space is at least two in the following way. Notice that for most fibers of the fibration $\mathcal{M}_{\left(A_{1}, \cdots, A_{m}\right)}^{v}\left(\Sigma, \mathcal{X}_{Z^{\alpha}}, J, \nu\right) \rightarrow Z^{\alpha}$, the boundary part of a fiber is of codimension two in the fiber as we know from the non-fiberwise case. Since $\overline{\mathcal{M}}_{A_{1}, \cdots, A_{m}}^{v, p}\left(\Sigma, \mathcal{X}_{Z^{\alpha}}, J, \nu\right)$ has the required smooth structure, its boundary has codimension at least two.

Remark 1.3. Since the moduli space has a canonically defined orientation in the non-fiberwise case, this orientation and the orientation of $Z^{\alpha}$ give a natural orientation of the moduli space $\mathcal{M}_{\left(A_{1}, \cdots, A_{m}\right)}^{v}\left(\Sigma, \mathcal{X}_{Z^{\alpha}}, J, \nu\right)$ in the fiberwise case.

Proposition 1.3. For a generic $(J, \nu) \in \mathcal{P}_{k-1, p}^{v}\left(\mathcal{X}_{Z}, \omega\right)$, and $D \in \mathcal{D}_{A, \Sigma}^{J, \nu}$, $\operatorname{dim} \mathcal{N}_{\bar{D}}^{v}\left(\Sigma, \mathcal{X}_{Z^{\alpha}}, J, \nu\right) \leq 2 C_{1}(V) \cdot A+2 n(1-g(\Sigma))-2 k_{\bar{D}}-2 s_{D}+\operatorname{dim} Z^{\alpha}$ where $k_{\bar{D}}$ is the number of bubble components of $D$ (not $\bar{D}$ ), and $s_{D}$ is the number of marked points which are also bubbling points.

Proof. Similar to Proposition 4.14 in [RT]. We omit it.

We want to prove another transversality result which will be used in defining the fiberwise mixed invariants later. Let $\left\{U_{i}\right\}_{i=1}^{c},\left\{W_{j}\right\}_{j=1}^{d}$ be two (possibly non-compact) families of smooth manifolds, and $L_{i}: U_{i} \rightarrow$ $\mathcal{X}_{Z}, M_{j}: W_{j} \rightarrow \mathcal{X}_{Z}$ be smooth maps. We require that the images all lie in one stratum, say $\mathcal{X}_{Z^{\alpha}}$. For each data $\bar{D}$, we know that $\mathcal{N}_{\bar{D}}^{v}\left(\Sigma, \mathcal{X}_{Z^{\alpha}}, J, \nu\right)$ is a smooth manifold for generic $(J, \nu)$. Consider the intersections of the components of $\operatorname{Im} f$ and $\operatorname{Im}\left(M_{j}\right)$, where $f \in \mathcal{N}_{\bar{D}}^{v}\left(\Sigma, \mathcal{X}_{Z^{\alpha}}, J, \nu\right)$ consists of principal components $\left(f_{i}\right)_{i=1}^{m}$ and bubble components $\left(f^{j}\right)_{j=1}^{k}$. Without loss 
of generality, we may assume that $\operatorname{Im}\left(M_{1}\right), \cdots, \operatorname{Im}\left(M_{p}\right)$ intersect the principal components $\operatorname{Im} f_{i_{s}}$ of $f, 1 \leq s \leq p$, and $\operatorname{Im} M_{p+1}, \cdots, \operatorname{Im} M_{d}$ intersect the bubble components $\operatorname{Im} f^{j_{s}}$ of $f, p+1 \leq s \leq d$, we call this an intersection pattern, and denote it by $T$. If $M_{i}$ intersect more than one component, we simply choose one of them. Let $C=\left(\Sigma ; x_{1}, \cdots, x_{c}\right)$ be a stable curve, and $X=\left(x_{1}, \cdots, x_{c}\right)$ be the set of marked points. We consider evaluation map

$$
\begin{aligned}
& e_{X, T} \times L \times M: \mathcal{N}_{\bar{D}}^{v}\left(\Sigma, \mathcal{X}_{Z^{\alpha}}, J, \nu\right) \times \prod_{s=1}^{p} \tilde{\Sigma}_{i_{s}} \times\left(S^{2}\right)^{d-p} \times U \times W \longrightarrow \\
& \quad \mathcal{X}_{Z^{\alpha}}^{c+d} \times \mathcal{X}_{Z^{\alpha}}^{c+d} \\
& e_{X, T}\left(f_{i}, f^{j} ; y_{1}, \cdots, y_{p} ; y_{p+1}, \cdots, y_{d}\right) \\
& \quad=\left(f\left(x_{1}\right), \cdots, f\left(x_{c}\right) ; f_{i_{1}}\left(y_{1}\right), \cdots, f_{i_{p}}\left(y_{p}\right) ; f^{j_{p+1}}\left(y_{p+1}\right), \cdots, f^{j_{d}}\left(y_{d}\right)\right),
\end{aligned}
$$

where $U=\prod_{i} U_{i}, W=\prod_{j} W_{j}, L=\prod_{i} L_{i}, M=\prod_{j} M_{j}, G=P G L_{2}, \Delta_{T}^{\alpha} \subset$ $\mathcal{X}_{Z^{\alpha}}^{c+d} \times \mathcal{X}_{Z^{\alpha}}^{c+d}$ is the diagonal corresponding to the intersection pattern $T$, and $\Delta_{T}^{\alpha}$ is defined similar to $\Delta_{Z^{\alpha}, \bar{D}}^{v}$. Note that $(f ; \cdots)$ is in $\left(e_{X, T} \times L \times M\right)^{-1}\left(\Delta_{T}^{\alpha}\right)$ if and only if $f\left(x_{i}\right) \in \operatorname{Im}\left(L_{i}\right), \operatorname{Im} f_{i_{s}} \cap \operatorname{Im}\left(M_{s}\right) \neq \emptyset, 1 \leq s \leq p$, and $\operatorname{Im} f^{j_{s}} \cap$ $\operatorname{Im}\left(M_{s}\right) \neq \emptyset, p+1 \leq s \leq d$.

Proposition 1.4. For a generic $(J, \nu) \in \mathcal{P}_{k-1, p}\left(\mathcal{X}_{Z}, \omega\right)$, any $D \in \mathcal{D}_{A, \Sigma}^{J, \nu}$, $\left(e_{X, T} \times L \times M\right)^{-1}\left(\Delta_{T}^{\alpha}\right)$ is a smooth manifold of dimension

$$
\begin{aligned}
& \operatorname{dim} \mathcal{N}_{\bar{D}}^{v}\left(\Sigma, \mathcal{X}_{Z^{\alpha}}, J, \nu\right)+2 d-\operatorname{codim} L-\operatorname{codim} M \\
& \leq 2 C_{1}(V) \cdot A+2 n(1-g(\Sigma))+\operatorname{dim} Z^{\alpha} \\
& \quad-2 k_{\bar{D}}-2 s_{D}+2 d-\operatorname{codim} L-\operatorname{codim} M,
\end{aligned}
$$

where $s_{D}, k_{\bar{D}}$ are defined as in Proposition 1.3, $\operatorname{codim} L_{i}=\operatorname{dim} \mathcal{X}_{Z^{\alpha}}-\operatorname{dim} U_{i}$, $\operatorname{codim} C=\sum_{i} \operatorname{codim} C_{i}, \operatorname{codim} M_{j}=\operatorname{dim} \mathcal{X}_{Z^{\alpha}}-\operatorname{dim} W_{j}$, and $\operatorname{codim} M=$ $\sum_{j} \operatorname{codim} M_{j}$. When the proposition is true, we say that $\mathcal{N}_{\bar{D}}\left(\Sigma, \mathcal{X}_{Z^{\alpha}}, J, \nu\right)$ is transversal to $L \times M$ for $(X, T)$, and such $(J, \nu)$ is good.

Proof. We use the notation $E_{\bar{D}}^{-1}\left(\Delta_{Z^{\alpha}, \bar{D}}^{v}\right)$ as defined at the end of proof of Proposition 1.2. All we need to show is that

$e_{(X, T, \mathcal{P})} \times L \times M: E_{\bar{D}}^{-1}\left(\Delta_{Z^{\alpha}, \bar{D}}^{v}\right) \times \prod_{s=1}^{p} \tilde{\Sigma}_{i_{s}} \times\left(S^{2}\right)^{d-p} \times U \times W \longrightarrow \mathcal{X}_{Z^{\alpha}}^{c+d} \times \mathcal{X}_{Z^{\alpha}}^{c+d}$

is transversal to $\Delta_{T}^{\alpha}$, where map $e_{(X, T, \mathcal{P})}$ is defined similarly to $e_{X, T}$. Since $E_{\bar{D}}^{-1}\left(\Delta_{Z^{\alpha}, \bar{D}}^{v}\right) \rightarrow \mathcal{P}_{k-1, p}\left(\mathcal{X}_{Z}, \omega\right)$ is a Fredholm map, according to the SardSmale Theorem, for a generic $(J, \nu), e_{X, T}$ is transversal to $\Delta_{T}^{\alpha}$. 
To prove $e_{(X, T, \mathcal{P})} \times L \times M$ is transversal to $\Delta_{T}^{\alpha}$, assume that

$$
\begin{gathered}
\left(f_{i}, f^{j}, J, \nu, y_{k}, u_{i}, w_{j}\right) \in\left(e_{(X, T, \mathcal{P})} \times L \times M\right)^{-1}\left(\Delta_{T}^{\alpha}\right), \\
f\left(x_{i}\right)=L_{i}\left(u_{i}\right), f_{i_{s}}\left(y_{s}\right)=M_{s}\left(w_{s}\right), 1 \leq s \leq p, f^{j_{s}}\left(y_{s}\right)=M_{s}\left(w_{s}\right),
\end{gathered}
$$
$p+1 \leq s \leq d$, we need to prove that for any $X_{i} \in T_{f\left(x_{i}\right)} \mathcal{X}_{Z^{\alpha}}, Y_{s} \in$ $T_{f_{i_{s}}\left(y_{s}\right)} \mathcal{X}_{Z^{\alpha}}, 1 \leq s \leq p$, and $Y_{s} \in T_{f^{j_{s}}\left(y_{s}\right)} \mathcal{X}_{Z^{\alpha}}, p+1 \leq s \leq d$, we can find a family of curves $\left(f_{i, t}, f_{t}^{j}, J_{t}, \nu_{t}, y_{s, t}\right) \in E_{\bar{D}}^{-1}\left(\Delta_{Z^{\alpha}, \bar{D}}^{v}\right) \times \prod_{s=1}^{p} \Sigma_{i_{s}}$ $\times_{G^{d-p}}\left(S^{2}\right)^{d-p}$ such that $\left.\frac{d f_{t}\left(x_{i}\right)}{d t}\right|_{t=0}=X_{i},\left.\frac{d f_{i_{s}, t}\left(y_{s, t}\right)}{d t}\right|_{t=0}=Y_{s}, 1 \leq s \leq p$, and $\left.\frac{d f_{t}^{j_{s}}\left(y_{s, t}\right)}{d t}\right|_{t=0}=Y_{s}, p+1 \leq s \leq d$. We achieve this by perturbing $\left(f_{i}, f^{j}, J, \nu, y_{k}\right)$ several times, each time we make one of the requirements satisfied without affecting other parts. The perturbation goes like the follows: suppose we want make $\left.\frac{d f_{i_{1}, t}\left(y_{1, t}\right)}{d t}\right|_{t=0}=Y_{1}$. If $Y_{1}$ is a fiber direction in $T_{f_{i_{1}}\left(y_{1}\right)} \mathcal{X}_{Z^{\alpha}}$, we then only need to perturb the curve within the fiber $V_{z}$, the perturbation being similar to the one in the proof of Proposition 1.2. The only new feature is that if $y_{1}$ is a double or cusp point of the domain of $f$, the pertubation has to keep the intersection pattern of the cusp curve, since otherwise the perturbed family will not be in $E_{\bar{D}}^{-1}\left(\Delta_{Z^{\alpha}, \bar{D}}^{v}\right)$. This can be done as follows: choose a curve $c_{t}$ in $V_{z}$ with $c_{0}=f_{i_{1}}\left(y_{1}\right)$ and $\left.\frac{d c_{t}}{d t}\right|_{t=0}=Y_{1}$. For any principal component intersecting at $f_{i_{1}}\left(y_{1}\right)$, say $f_{i}$, by perturbing the fiberwise inhomgeneous term, we can find $f_{i, t}$ which is a local perturbation of $f_{i}$ around $y_{1}$, such that $f_{i, t}\left(y_{1}\right)=c_{t}$. For any bubble component intersecting at $f_{i_{1}}\left(y_{1}\right)$, say $f^{j}$, by the work of D. McDuff [M2], we can actually perturb $J$ and $\nu$ in the same way as in Proposition 1.2, with $f_{t}^{j}\left(y_{1}\right)=c_{t}$. Now the differnt components of map $f_{t}$ intersect at point $c_{t}$. Once again we can extend the perturbed $\left(\left.J\right|_{V_{z}}\right)_{t},\left(\left.\nu\right|_{V_{z}}\right)_{t}$ to $\left(J_{t}, \nu_{t}\right) \in \mathcal{P}_{k-1, p}\left(\mathcal{X}_{Z}, \omega\right)$.

If $Y_{1}$ is not a fiber direction, we choose a trivialization of $\mathcal{X}_{Z^{\alpha}}$ around $z \in$ $Z^{\alpha}$ (see Remark 1.2). We only study the case that $Y_{1}$ is a horizontal vector under the trivialization, since this is sufficient for proving the surjectivity. Choose a curve $z_{t}$ in $Z^{\alpha}$ with $z_{0}=z,\left.\frac{d z_{t}}{d t}\right|_{t=0}=Y_{1}$, we perturb $J$ and $\nu$ in the following way: $\left.\left.J_{t}\right|_{V_{z_{t}}} \cong J\right|_{V_{z}},\left.\left.\nu_{t}\right|_{V_{z_{t}}} \cong \nu\right|_{V_{z}}$, so take $f_{t}=f$ but view that $\operatorname{Im} f_{t}$ lies in the fiber $V_{z_{t}}$. Now $f_{i_{1}, t}\left(y_{1, t}\right)=\left(z_{t}\right.$, fiber part $)$, the horizontal part of $\left.\frac{d f_{i_{1, t}}\left(y_{1, t}\right)}{d t}\right|_{t=0}$ is $Y_{1}$ and this perturbation keeps the intersection pattern of the cusp curve. So $e_{(X, T, \mathcal{P})} \times L \times M$ is transversal to $\Delta_{T}^{\alpha}$.

Dimension counting being routine, the inequality follows from Proposition 1.3 , and the proposition is proved.

Remark 1.4. (i) If $\tilde{Z}$ is a submanifold of $Z^{\alpha}$, assume that $\left(J_{0}, \nu_{0}\right)$ is good for $\mathcal{N}_{\bar{D}}^{v}\left(\Sigma, \mathcal{X}_{\tilde{Z}}, J_{0}, \nu_{0}\right)$, and consider the subspace $\widetilde{\mathcal{P}}_{k-1, p}\left(\mathcal{X}_{Z}, \omega\right) \subset$ 
$\mathcal{P}_{k-1, p}\left(\mathcal{X}_{Z}, \omega\right)$ which consists of $(J, \nu)$ 's whose restriction to $\mathcal{X}_{\tilde{Z}}$ equals $\left(J_{0}, \nu_{0}\right)$. Then using this space instead of $\mathcal{P}_{k-1, p}\left(\mathcal{X}_{Z}, \omega\right)$ in the proofs of Proposition 1.2 and 1.3, one can show that for a generic $(J, \nu)$ of $\widetilde{\mathcal{P}}_{k-1, p}\left(\mathcal{X}_{Z}, \omega\right), \mathcal{N}_{\bar{D}}^{v}\left(\Sigma, \mathcal{X}_{Z^{\alpha}}, J, \nu\right)$ is smooth and transversal to $L \times M$, since we may perturb $(J, \nu)$ only outside of $\mathcal{X}_{\tilde{Z}}$.

(ii) If $j_{t}$ is a smooth family of complex structure on $\Sigma, t \in[0,1]$, denote by $\Sigma_{t} \Sigma$ equipped with $j_{t}$, then one can consider the space $\cup_{t} \mathcal{N}_{\bar{D}}^{v}\left(\Sigma_{t}, \mathcal{X}_{Z^{\alpha}}, J, \nu\right)$. For a generic $(J, \nu) \in \mathcal{P}_{k-1, p}\left(\mathcal{X}_{Z}, \omega\right)$, this space is smooth and transversal to $L \times M$. The reason is: in the proof of Proposition 1.2 and 1.3, replacing those $M a p$ and $E_{\bar{D}}^{-1}\left(\Delta_{Z^{\alpha}, \bar{D}}^{v}\right)$ spaces by their union over $t, t \in[0,1]$, the remaining argument is the same.

The following proposition about the transversality of components of fiberwise cusp curves is important for the gluing argument in section 3 . Adopting the notation of Proposition 1.2, consider the moduli space

$$
\mathcal{M}_{\left(A_{1}, \cdots, A_{m}\right)}^{v}\left(\Sigma, \mathcal{X}_{Z^{\alpha}}, J, \nu\right) \text {. }
$$

It consists of fiberwise $(J, \nu)$ perturbed holomorphic maps which have no bubbles and whose components intersect each other at double points. We would like to show that the subset where at least two components intersect non-transversally is of codimension 2. Without loss of generality, we can assume that $\Sigma$ has only two components $\Sigma_{1}$ and $\Sigma_{2}$. Let $y_{1}$ and $y_{2}$ be the distinguished points on $\Sigma_{1}$ and $\Sigma_{2}$ corresponding to the intersection. We allow a self-intersection by setting $\Sigma_{1}=\Sigma_{2}$ and using their smooth resolution below. We then define

$$
\begin{aligned}
e_{y_{1}, y_{2}}: \mathcal{M}_{\left(A_{1}, A_{2}\right)}^{v}\left(\Sigma, \mathcal{X}_{Z^{\alpha}}, J, \nu\right) \rightarrow & \\
& \operatorname{Hom}\left(T_{y_{1}} \Sigma_{1},\left.E\right|_{\mathcal{X}_{Z^{\alpha}}}\right) \oplus \operatorname{Hom}\left(T_{y_{2}} \Sigma_{2},\left.E\right|_{\mathcal{X}_{Z^{\alpha}}}\right) \\
e_{y_{1}, y_{2}}(f)= & \left(d f_{1}\left(y_{1}\right), d f_{2}\left(y_{2}\right)\right),
\end{aligned}
$$

where $f_{1} \in \mathcal{M}_{A_{1}}\left(\Sigma_{1}, \mathcal{X}_{Z^{\alpha}}, J, \nu_{1}\right), f_{2} \in \mathcal{M}_{A_{2}}\left(\Sigma_{2}, \mathcal{X}_{Z^{\alpha}}, J, \nu_{2}\right)$. A generic element in $\operatorname{Hom}\left(T_{y_{1}} \Sigma_{1}, E{\mid \mathcal{X}_{Z^{\alpha}}}\right) \oplus \operatorname{Hom}\left(T_{y_{2}} \Sigma_{2},\left.E\right|_{\mathcal{X}_{Z^{\alpha}}}\right)$ is a smooth fibration over $\mathcal{X}_{Z^{\alpha}}$ of dimension $10 n+\operatorname{dim} Z^{\alpha}$ and maximal rank 4 . If $f_{1}$ and $f_{2}$ do not intersect transversally, then $f$ 's image will have lower rank. The set of homomorphisms of lower ranks is a union of manifolds $R_{i}$ consisting of homomorphisms of rank $i(\mathrm{i}=0,1,2,3) . \operatorname{dim} R_{0}=2 n+\operatorname{dim} Z^{\alpha}, \operatorname{dim} R_{i}=2(i+1) n+$ $\operatorname{dim} Z^{\alpha}+4-i, \mathrm{i}=1,2,3$. Thus their codimensions are $8 n, 6 n-3,4 n-2,2 n-1$.

Proposition 1.5. For a generic $(J, \nu), e_{y_{1}, y_{2}}$ is transversal to $R_{i}$. Hence $e_{y_{1}, y_{2}}^{-1}\left(R_{i}\right)$ has codimension at least $2 n-1$ in $\mathcal{M}_{\left(A_{1}, A_{2}\right)}^{v}\left(\Sigma, \mathcal{X}_{Z^{\alpha}}, J, \nu\right)$, $i \stackrel{=}{=} 0,1,2,3$. 
Proof. Use the notation $E_{\bar{D}}^{-1}\left(\Delta_{Z^{\alpha}, \bar{D}}^{v}\right)$ as defined at the end of the proof of Proposition 1.2. Now choose data $\bar{D}$ with no bubble components and denote $E_{\bar{D}}^{-1}\left(\Delta_{Z^{\alpha}, \bar{D}}^{v}\right)$ by $\mathcal{M}_{\left(A_{1}, A_{2}\right)}^{v}\left(\Sigma, \mathcal{X}_{Z^{\alpha}}, \mathcal{P}\right)$. Consider the map

$$
\begin{aligned}
& E_{y_{1}, y_{2}}: \mathcal{M}_{\left(A_{1}, A_{2}\right)}^{v}\left(\Sigma, \mathcal{X}_{Z^{\alpha}}, \mathcal{P}\right) \rightarrow \operatorname{Hom}\left(T_{y_{1}} \Sigma_{1},\left.E\right|_{\mathcal{X}_{Z^{\alpha}}}\right) \oplus \operatorname{Hom}\left(T_{y_{2}} \Sigma_{2},\left.E\right|_{\mathcal{X}_{Z^{\alpha}}}\right) \\
& E_{y_{1}, y_{2}}(f, J, \nu)=\left(d f_{1}\left(y_{1}\right), d f_{2}\left(y_{2}\right)\right),
\end{aligned}
$$

we can show that $E_{y_{1}, y_{2}}$ is transversal to $R_{i}, i=0,1,2,3$. The proof, similar to the proof of proposition 1.2, is omitted(see [RT], Theorem 5.10).

\section{Fiberwise Gromov-Witten Mixed Invariants.}

We now begin the definition of fiberwise mixed invariants, our aim being to use it to define the fiberwise quantum cohomology. Let $C=$ $\left(\Sigma ; x_{1}, \cdots, x_{c}\right)$ be a stable curve of genus $g, \Sigma=\left(\Sigma_{1}, \cdots, \Sigma_{m}\right), X=$ $\left(x_{1}, \cdots, x_{c}\right)$, and $A_{1}, \cdots, A_{m}$ be homology classes in $H_{2}(V ; \mathbb{Z}), A=A_{1}+$ $\cdots+A_{m}$. Without loss of generality, assume that $x_{1}, \cdots, x_{i_{1}}$ lie in $\Sigma_{1}$, $x_{i_{1}+1}, \cdots, x_{i_{2}}$ lie in $\Sigma_{2}, \cdots$, and $x_{i_{m-1}+1}, \cdots, x_{i_{m}}$ lie in $\Sigma_{m}$. Denote this position pattern by $P_{1}$. Take a bundle $\mathcal{X} \stackrel{p}{\rightarrow} Y$ satisfying Assumption ( $(*)$. Let $\left\{\alpha_{i}\right\}_{1}^{c},\left\{\beta_{j}\right\}_{1}^{d}$ be rational cohomology classes of $\mathcal{X}$, and $\gamma$ be the rational homology class of $Y$ satisfying

$$
\sum_{1}^{c} \operatorname{deg} \alpha_{i}+\sum_{1}^{d}\left(\operatorname{deg} \beta_{j}-2\right)=2 C_{1}(V) \cdot A+2 n(1-g)+\operatorname{deg}(\gamma) .
$$

Here we work with rational coefficients for homology and cohomology, but we could use real or complex coefficients. In section 5 we will actually use complex coefficients. Integer coefficients can not be used because an integral homology class may have no pseudo-manifold representative.

Let $F: Z \rightarrow Y$ be a pseudo-manifold representative of a homology class $\gamma$. Using the notation after Assumption $(\star), \tilde{F}^{*}\left(\alpha_{i}\right)$ and $\tilde{F}^{*}\left(\beta_{j}\right)$ are then cohomology classes in the pseudo-manifold $\mathcal{X}_{Z}$. Denote their Poincare duals by $P D\left(\tilde{F}^{*}\left(\alpha_{i}\right)\right)$ and $P D\left(\tilde{F}^{*}\left(\beta_{j}\right)\right)$ respectively. We can represent $P D\left(\tilde{F}^{*}\left(\alpha_{i}\right)\right)$ and $P D\left(\tilde{F}^{*}\left(\beta_{j}\right)\right)$ by pseudo-manifolds $U_{i}$ and $W_{j}$ in $\mathcal{X}_{Z}$ in the following way: there are continuous maps

$$
L_{i}: U_{i} \rightarrow \mathcal{X}_{Z}, \quad M_{j}: W_{j} \rightarrow \mathcal{X}_{Z}
$$

such that on each stratum, say $U_{i}^{s}$ or $W_{j}^{t}$, both $L_{i}$ and $M_{j}$ are smooth and each image $L_{i}\left(U_{i}^{s}\right)$ or $M_{j}\left(W_{j}^{t}\right)$ lies entirely in some stratum of $\mathcal{X}_{Z}$, say $\mathcal{X}_{Z^{\alpha_{i, s}}}$ or $\mathcal{X}_{Z^{\beta_{j, t}}}$. Furthermore they satisfy the following general position condition. 


\section{General position condition:}

$$
\begin{aligned}
& \operatorname{dim} \mathcal{X}_{Z^{\alpha_{i, s}}}-\operatorname{dim} U_{i}^{s} \geq \operatorname{dim} \mathcal{X}_{Z}-\operatorname{dim} U_{i}, \\
& \operatorname{dim} \mathcal{X}_{Z^{\beta_{j, t}}}-\operatorname{dim} W_{j}^{t} \geq \operatorname{dim} \mathcal{X}_{Z}-\operatorname{dim} W_{j} .
\end{aligned}
$$

Note that if $Z$ is smooth, then this definition coincides with the ordinary definition of the pseudo-manifold representative of a rational homology class.

Denote $L=\prod_{i} L_{i}, M=\prod_{j} M_{j}, L \times M: \prod_{i} U_{i} \times \prod_{j} W_{j} \rightarrow \mathcal{X}_{Z}^{c+d}$. Denote by $\operatorname{Im}$ the image of a map, fix an intersection pattern $\mathrm{T}: \operatorname{Im} f_{s_{j}}$ intersects $\operatorname{Im} W_{j}, j=1, \cdots, d$. Then define the evaluation map

$$
\begin{aligned}
& e_{(\Sigma, P, J, \nu)}: \mathcal{M}_{\left(A_{1}, \cdots, A_{m}\right)}^{v}\left(\Sigma, \mathcal{X}_{Z}, J, \nu\right) \times \prod_{j=1}^{d} \Sigma_{s_{j}} \longrightarrow \mathcal{X}_{Z}^{c+d} \\
& \left(f ; y_{1}, \cdots, y_{d}\right) \longrightarrow\left(f\left(x_{1}\right), \cdots, f\left(x_{c}\right) ; f_{s_{1}}\left(y_{1}\right), \cdots, f_{s_{d}}\left(y_{d}\right)\right) .
\end{aligned}
$$

Note that $e_{(\Sigma, P, J, \nu)}$ is a smooth map. It follows from our assumption on the degree of $\alpha_{i}$ and $\beta_{j}$ that the image of $e_{(\Sigma, P, J, \nu)}$ and $L \times M$ have complementary dimensions in $\mathcal{X}_{Z}^{c+d}$. Moreover, we have

Proposition 2.1. Under assumption (2.1), for a generic fiberwise almost complex structure $J$ and a generic inhomogeneous term $\nu$ in $\mathcal{P}_{k-1, p}\left(\mathcal{X}_{Z}, \omega\right)$, we have

(i) There are no sequences $\left\{f_{r}\right\}_{1}^{\infty}$ in $\mathcal{M}_{\left(A_{1}, \cdots, A_{m}\right)}^{v}\left(\Sigma, \mathcal{X}_{Z}, J, \nu\right)$ such that $f_{r}\left(x_{i}\right)$ converges to a point in $L_{i}\left(U_{i}\right)$ as $r \rightarrow \infty, f_{r, s_{j}}\left(\Sigma_{s_{j}}\right) \cap M_{j}\left(W_{j}\right) \neq$ $\emptyset$ and at least one of the following is true:

(a) for some $i, f_{r}\left(x_{i}\right)$ converges to a point in $L_{i}\left(U_{i} \backslash U_{i}^{0}\right)$,

(b) for some $j, f_{r, s_{j}}\left(\Sigma_{s_{j}}\right) \cap M_{j}\left(W_{j} \backslash W_{j}^{0}\right) \neq \emptyset$.

Here $U_{i}^{0}$ and $W_{j}^{0}$ denote the top strata in $U_{i}$ and $W_{j}$ respectively.

(ii) There are no sequences $\left\{f_{r}\right\}_{1}^{\infty}$ in $\mathcal{M}_{\left(A_{1}, \cdots, A_{m}\right)}^{v}\left(\Sigma, \mathcal{X}_{Z}, J, \nu\right)$ with $f_{r}\left(x_{i}\right)$ converges to a point in $L_{i}\left(U_{i}\right)$ as $r \rightarrow \infty, f_{r, s_{j}}\left(\Sigma_{s_{j}}\right) \cap M_{j}\left(W_{j}\right) \neq \emptyset$ and the limit $f$ in not in $\mathcal{M}_{\left(A_{1}, \cdots, A_{m}\right)}^{v}\left(\Sigma, \mathcal{X}_{Z^{0}}, J, \nu\right)$. Here $Z^{0}$ denotes the top stratum in $Z$.

(iii) $e_{(\Sigma, P, J, \nu)}\left(\mathcal{M}_{\left(A_{1}, \cdots, A_{m}\right)}^{v}\left(\Sigma, \mathcal{X}_{Z^{0}}, J, \nu\right)\right)$ and $L \times M\left(U^{0} \times W^{0}\right)$ intersect transversally at finitely many points, i.e., there are finitely many

$$
\left(f ; y_{1}, \cdots, y_{d}\right) \in \mathcal{M}_{\left(A_{1}, \cdots, A_{m}\right)}^{v}\left(\Sigma, \mathcal{X}_{Z^{0}}, J, \nu\right) \times \prod_{j=1}^{d} \Sigma_{s_{j}},(u, w) \in U^{0} \times W^{0}
$$


such that $e_{(\Sigma, P, J, \nu)}\left(f ; y_{1}, \cdots, y_{d}\right)=(L(u), M(w))$, and at each intersection point, the image of the tangent space $T_{(u, w)} U^{0} \times W^{0}$ under $L \times M$ is transverse to the image of the tangent space

$$
T_{\left(f ; y_{1}, \cdots, y_{d}\right)}\left(\mathcal{M}_{\left(A_{1}, \cdots, A_{m}\right)}\left(\Sigma, \mathcal{X}_{Z^{0}}, J, \nu\right) \times \prod_{j=1}^{d} \Sigma_{s_{j}}\right)
$$

under the evaluation map.

Proof.

(i) If this fails, by Proposition 1.1 (here we adopt the notation there), there is a cusp curve $f \in \mathcal{N}_{\bar{D}}^{v}\left(\Sigma, \mathcal{X}_{Z}, J, \nu\right)$ for some $\bar{D}$ satisfying: (1) $f(\tilde{\Sigma}) \cap \operatorname{Im}\left(M_{j}\right) \neq \emptyset, 1 \leq j \leq d$; (2) for each marked point $x_{i}, i=1, \cdots, c$, either $f\left(x_{i}\right) \in \operatorname{Im}\left(L_{i}\right)$ or a bubble occurs at $x_{i}$. In the second case, $f\left(x_{i}\right)$ may not be in $\operatorname{Im}\left(L_{i}\right)$, but $\operatorname{Im}\left(L_{i}\right)$ will intersect a bubble tree coming out of $x_{i}$. Note that $\operatorname{Im}\left(M_{j}\right)$ may intersect a bubble instead of the principal component of $f$. Therefore we see that $f$ could have fewer marked points and the number of homology classes corresponding to the unmarked part increases. Let $X^{\prime} \subset X$ be the subset of marked points which are not bubbling points. Suppose that $X^{\prime}=\left(x_{1}, \cdots, x_{p}\right)$, then there are at least $c-p$ bubbles. $c+d-p$ is the number of pseudo-manifolds in $P D\left(\tilde{F}^{*}\left(\alpha_{i}\right)\right), P D\left(\tilde{F}^{*}\left(\beta_{j}\right)\right)$ which intersect $\operatorname{Im}(f)$. Let $f\left(x_{i}\right) \in L_{i}\left(U_{i}\right)$ for $i=1, \cdots, p, \operatorname{Im}(f) \cap L_{i}\left(U_{i}\right) \neq \emptyset$, for $i=p+1, \cdots, c$, and $\operatorname{Im}(f) \cap M_{j}\left(W_{j}\right) \neq \emptyset$ for $j=1, \cdots, d$. Suppose that $f$ intersects these pseudo-manifolds in a intersection pattern $T$, implying

$$
\left(e_{X, T} \times L \times M\right)^{-1}\left(\Delta_{T}^{\alpha}\right) \neq \emptyset
$$

where $\operatorname{Im}(f) \subset \mathcal{X}_{Z^{\alpha}}$ for some $\alpha$ and $e_{X, T}$ is defined before Proposition 1.4. Proposition 1.4 says that $\left(e_{X, T} \times L \times M\right)^{-1}\left(\Delta_{T}^{\alpha}\right)$ is a smooth manifold, we have an estimate of its dimension

$$
\begin{aligned}
\operatorname{dim}\left(e_{X, T} \times L \times M\right)^{-1}\left(\Delta_{T}^{\alpha}\right) \\
=\operatorname{dim} \mathcal{N}_{\bar{D}}^{v}\left(\Sigma, \mathcal{X}_{Z^{\alpha}}, J, \nu\right)+2(c+d-p)-\operatorname{codim} U_{i}^{s(\alpha)}-\operatorname{codim} W_{j}^{t(\alpha)} \\
=2 C_{1}(V) \cdot A+2 n(1-g)-2(c-p)+2(c+d-p) \\
\quad-\operatorname{codim} U_{i}^{s(\alpha)}-\operatorname{codim} W_{j}^{t(\alpha)}+\operatorname{dim} Z^{\alpha},
\end{aligned}
$$

where $U_{i}^{s(\alpha)}$ stands for all the stratum in $U_{i}$ whose image lies in $\mathcal{X}_{Z^{\alpha}}$ and $W_{j}^{t(\alpha)}$ has a similar meaning. If $\alpha=0$, by the assumption we have 


$$
\begin{aligned}
& \operatorname{codim} U_{i}^{s(\alpha)} \geq \operatorname{codim} U_{i}+2 \text { or } \operatorname{codim} W_{j}^{t(\alpha)} \geq \operatorname{codim} W_{j}+2, \text { so } \\
& \operatorname{dim}\left(e_{X, T} \times L \times M\right)^{-1}\left(\Delta_{T}^{0}\right) \\
& \leq 2 C_{1}(V) \cdot A+2 n(1-g)+2 d-\operatorname{codim} U_{i}-\operatorname{codim} W_{j}-2+\operatorname{dim} Z^{0} \\
& =-2
\end{aligned}
$$

If $\alpha \neq 0$, then the general position condition and $\operatorname{dim} Z^{\alpha} \leq \operatorname{dim} Z^{0}-2$ imply

$$
\begin{aligned}
& \operatorname{dim}\left(e_{X, T} \times L \times M\right)^{-1}\left(\Delta_{T}^{\alpha}\right) \\
& \leq 2 C_{1}(V) \cdot A+2 n(1-g)+2 d-\operatorname{codim} U_{i}-\operatorname{codim} W_{j}+\operatorname{dim} Z^{0}-2 \\
& =-2 .
\end{aligned}
$$

So in either case, we get a contradiction. (i) is proved.

(ii) The first half is the same as the proof of (i), the only modification is the dimension counting.

$$
\begin{aligned}
& \operatorname{dim}\left(e_{X, T} \times L \times M\right)^{-1}\left(\Delta_{T}^{\alpha}\right) \\
& =\operatorname{dim} \mathcal{N}_{\bar{D}}^{v}\left(\Sigma, \mathcal{X}_{Z^{\alpha}}, J, \nu\right)+2(c+d-p)-\operatorname{codim} U_{i}^{s(\alpha)}-\operatorname{codim} W_{j}^{t(\alpha)} \\
& \leq 2 C_{1}(V) \cdot A+2 n(1-g)-2-2(c-p)+2(c+d-p) \\
& \quad-\operatorname{codim} U_{i}^{s(\alpha)}-\operatorname{codim} W_{j}^{t(\alpha)}+\operatorname{dim} Z^{\alpha} \\
& \leq-2,
\end{aligned}
$$

where we use that $D$ has a bubble component to get -2 in the second to last step.

This a contradiction. (ii) is proved.

(iii) Considering the restriction of $e_{(\Sigma, P, J, \nu)}$ to $\mathcal{M}_{\left(A_{1}, \cdots, A_{m}\right)}^{v}\left(\Sigma, \mathcal{X}_{Z^{0}}, J, \nu\right) \times$ $\prod_{j=1}^{d} \Sigma_{s_{j}}$ and the restriction of $L \times M$ to $U^{0} \times W^{0}$, (iii) follows from Proposition 1.4.

Remark 2.1. (i) Note that if each of $\operatorname{dim} Z, \operatorname{dim} U_{i}$, and $\operatorname{dim} W_{j}$ is one dimension higher than it is in Proposition 2.1. It is still correct, except that we should modify (iii) as follows: their intersection is a one dimensional smooth manifold in $\mathcal{M}_{\left(A_{1}, \cdots, A_{m}\right)}^{v}\left(\Sigma, \mathcal{X}_{Z^{0}}, J, \nu\right) \times U^{0} \times W^{0}$. The reason is: in the proof of the above proposition, if the dimensions of $Z, U_{i}$, and $W_{j}$ are raised by 1 , the result of dimension counting in (i) and (ii) is -1 , which still gives a contradiction. 
(ii) Assume $\operatorname{dim} Z, \operatorname{dim} U_{i}$, and $\operatorname{dim} W_{j}$ are one dimension higher than it is in Proposition 2.1, and $Z, U_{i}, W_{j}$ have submanifolds $\tilde{Z}, \tilde{U}_{i}, \tilde{W}_{j}$, respectively. According to Remark 1.4 (i), Proposition 2.1 is still correct even if we fixed $(J, \nu)$ 's value on $\tilde{Z}, \tilde{U}_{i}, \tilde{W}_{j}$ to be good. If $\tilde{Z}, \tilde{U}_{i}, \tilde{W}_{j}$ are boundaries, the one dimensional manifold in i) has boundary, this essentially giving a cobordism.

(iii) Suppose that $Z$ satisfies (2.1), if we consider the moduli space appearing in Remark 1.4 ii), Proposition 2.1 is still correct. The image of the moduli space $\cup_{t} \mathcal{M}_{\left(A_{1}, \cdots, A_{m}\right)}^{v}\left(\Sigma_{t}, \mathcal{X}_{Z^{0}}, J, \nu\right) \times \prod_{j=1}^{d} \Sigma_{s_{j}}$ will only intersect the image of $U^{0} \times W^{0}$, and the intersection will be transverse giving a smooth one dimensional manifold.

Now we define the fiberwise mixed invariants as follows. Denote by $P:=\left(P_{1}, T\right)$ the pair: the position pattern $P_{1}$ and intersection pattern $T$ and denote $e_{X, T}$ by $e_{(\Sigma, P, J, \nu)}$. Fix a pair $(J, \nu)$ such that $e_{(\Sigma, P, J, \nu)}$ and $\mathcal{M}_{\left(A_{1}, \cdots, A_{m}\right)}^{v}\left(\Sigma, \mathcal{X}_{Z}, J, \nu\right)$ satisfy all the properties in Proposition 2.1. Again we will call such $(J, \nu)$ good. First we associate a multiplicity $m(f)$ to each $f$ in $\mathcal{M}_{\left(A_{1}, \cdots, A_{m}\right)}^{v}\left(\Sigma, \mathcal{X}_{Z}, J, \nu\right)$. We define $m(f)$ to be zero if either $f\left(x_{i}\right)$ is not in $L_{i}\left(U_{i}\right)$ for some $i$, or $f_{s_{j}}\left(\Sigma_{s_{j}}\right)$ does not intersect with one of the $M_{j}\left(W_{j}\right)$ for some $j$. If $f$ is given as in Proposition 2.1, there are finitely many $\left(y_{t 1}, \cdots, y_{t d}\right)(1 \leq t \leq l)$ such that $f_{s_{j}}\left(y_{t j}\right) \in M_{j}\left(W_{j}^{0}\right)$. Putting $\epsilon(f, t)$ to be \pm 1 , the sign being determined by the orientation of $\mathcal{M}_{\left(A_{1}, \cdots, A_{m}\right)}^{v}\left(\Sigma, \mathcal{X}_{Z^{0}}, J, \nu\right) \times \prod_{j=1}^{d} \Sigma_{s_{j}}$ at $\left(f ; y_{t 1}, \cdots, y_{t d}\right)$, the orientation of $U^{0} \times W^{0}$ at $(u, w)$, the orientation of $\mathcal{X}_{Z^{0}}^{c+d}$ at $\left(f\left(x_{1}\right), \cdots, f\left(x_{c}\right), f_{s_{1}}\left(y_{t 1}\right), \cdots, f_{s_{d}}\left(y_{t d}\right)\right)$, and the Jacobian of the maps $e_{(\Sigma, P, J, \nu)}$ at $L \times M$. We define

$$
m(f)=\sum_{t=1}^{l} \epsilon(f, t) .
$$

Finally we define the fiberwise mixed invariant

$$
\Phi_{\left(A_{1}, \cdots, A_{m}, \omega, \Sigma, P\right)}^{v}\left(\alpha_{1}, \cdots, \alpha_{c} \mid \beta_{1}, \cdots, \beta_{d}\right)(\gamma)=\sum m(f) .
$$

For convenience, we define

$$
\Phi_{\left(A_{1}, \cdots, A_{m}, \omega, \Sigma, P\right)}^{v}\left(\alpha_{1}, \cdots, \alpha_{c} \mid \beta_{1}, \cdots, \beta_{d}\right)(\gamma)=0,
$$

in case that

$$
\sum_{1}^{c} \operatorname{deg}\left(\alpha_{i}\right)+\sum_{1}^{d}\left(\operatorname{deg}\left(\beta_{j}\right)-2\right) \neq 2 C_{1}(V) \cdot A+2 n(1-g)+\operatorname{deg}(\gamma) .
$$


This defines a map

$$
\Phi_{\left(A_{1}, \cdots, A_{m}, \omega, \Sigma, P\right)}^{v}: \prod H^{*}(\mathcal{X} ; \mathbb{Q}) \times \prod H^{*}(\mathcal{X} ; \mathbb{Q}) \rightarrow H^{*}(Y ; \mathbb{Q}) .
$$

Note that when $Y$ is a point, $\mathcal{X}=V$, the fiberwise mixed invariants coincide with the mixed invariants defined by Ruan and Tian in [RT].

The following proposition assures that

$$
\Phi_{\left(A_{1}, \cdots, A_{m}, \omega, \Sigma, P\right)}^{v}\left(\alpha_{1}, \cdots, \alpha_{c} \mid \beta_{1}, \cdots, \beta_{d}\right)(\gamma)
$$

is indeed a symplectic invariant.

Proposition 2.2. $\Phi_{\left(A_{1}, \cdots, A_{m}, \omega, \Sigma, P\right)}^{v}\left(\alpha_{1}, \cdots, \alpha_{c} \mid \beta_{1}, \cdots, \beta_{d}\right)(\gamma)$ is independent of the choice of $(J, \nu)$; the marked points $x_{1}, \cdots, x_{c}$ of the same position pattern $P_{1}$ in $\Sigma$; the conformal structure on $\Sigma$; the choice of pseudomanifolds $\left(L_{i}, U_{i}\right),\left(M_{j}, W_{j}\right),(F, Z)$ representing $\alpha_{i}, \beta_{j}, \gamma(i=1, \cdots, c ; j=$ $1, \cdots, d)$. Furthermore, the invariant depends only on the semi-positive deformation class of $\omega$.

Proof. In the proof we adopt the notation used in proposition 2.1, and divide the proof into several parts.

(i) We show that $\Phi_{\left(A_{1}, \cdots, A_{m}, \omega, \Sigma, P\right)}^{v}\left(\alpha_{1}, \cdots, \alpha_{c} \mid \beta_{1}, \cdots, \beta_{d}\right)(\gamma)$ is independent of the choice of good $(J, \nu)$. Suppose $\left(J_{0}, \nu_{0}\right),\left(J_{1}, \nu_{1}\right)$ are two choices, denote $\tilde{Z}=Z \times[0,1]$, define $\mathcal{X}_{\tilde{Z}} \rightarrow \tilde{Z}$ to be a fiber bundle with fiber $V$ induced from $\mathcal{X}_{Z}$, the fiberwise symplectic form $\tilde{\omega}$ on $\mathcal{X}_{\tilde{Z}}$ being also the induced one. Note that $\mathcal{X}_{\tilde{Z}} \rightarrow \tilde{Z}$ satisfies Assumption $(\star)$. Define $\mathcal{P}_{k-1, p}^{v}\left(\mathcal{X}_{\tilde{Z}}, \tilde{\omega}\right)$ similarly to $\mathcal{P}_{k-1, p}^{v}\left(\mathcal{X}_{Z}, \omega\right)$, but with a boundary condition: for any $(\tilde{J}, \tilde{\nu}) \in \mathcal{P}_{k-1, p}^{v}\left(\mathcal{X}_{\tilde{Z}}, \tilde{\omega}\right),\left.(\tilde{J}, \tilde{\nu})\right|_{\mathcal{X}_{Z \times 0}} \cong$ $\left(J_{0}, \nu_{0}\right),\left.(\tilde{J}, \tilde{\nu})\right|_{\mathcal{X}_{Z \times 1}} \cong\left(J_{1}, \nu_{1}\right)$. Then choose $\tilde{L}_{i}=L_{i} \times i d, \tilde{M}_{j}=$

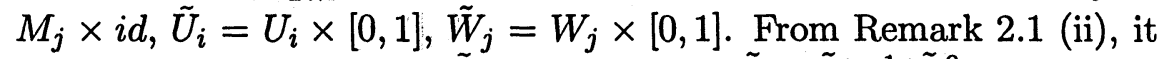
follows that for a generic $(\tilde{J}, \tilde{\nu}),\left(e_{(\Sigma, P, \tilde{J}, \tilde{\nu})} \times \tilde{L} \times \tilde{M}\right)^{-1}\left(\tilde{\Delta}_{T}^{0}\right)$ will give a one dimensional cobordism between $\left(e_{\left(\Sigma, P, J_{0}, \nu_{0}\right)} \times L \times M\right)^{-1}\left(\Delta_{T}^{0}\right)$ and $\left(e_{\left(\Sigma, P, J_{1}, \nu_{1}\right)} \times L \times M\right)^{-1}\left(\Delta_{T}^{0}\right)$ in the space $\mathcal{M}_{\left(A_{1}, \cdots, A_{m}\right)}^{v}\left(\Sigma, \mathcal{X}_{\tilde{Z}}, \tilde{J}, \tilde{\nu}\right) \times$ $\prod_{j=1}^{d} \Sigma_{s_{j}} \times \tilde{U}^{0} \times \tilde{W}^{0}$. This cobordism tell us that $\Phi_{\left(A_{1}, \cdots, A_{m}, \omega, \Sigma, P\right)}^{v}\left(\alpha_{1}\right.$, $\left.\cdots, \alpha_{c} \mid \beta_{1}, \cdots, \beta_{d}\right)(\gamma)$ is independent of the choice $(J, \nu)$.

(ii) We show that $\Phi_{\left(A_{1}, \cdots, A_{m}, \omega, \Sigma, P\right)}^{v}\left(\alpha_{1}, \cdots, \alpha_{c} \mid \beta_{1}, \cdots, \beta_{d}\right)(\gamma)$ is independent of the choice of the conformal structure $j$ on $\Sigma$. Let $j_{0}$ and $j_{1}$ be two conformal structure on $\Sigma$. We can connect them by a family of 
conformal structures $j_{t}, t \in[0,1]$. From Remark 2.1 (iii), for a generic $(J, \nu)$, the image of the moduli space $\cup_{t} \mathcal{M}_{\left(A_{1}, \cdots, A_{m}\right)}^{v}\left(\Sigma_{t}, \mathcal{X}_{Z^{0}}, J, \nu\right) \times$ $\prod_{j=1}^{d} \Sigma_{s_{j}}$ will only intersect the image of $U^{0} \times W^{0}$, the intersection will be transverse and give a smooth one dimensional manifold. But this one dimensional manifold is the cobordism between $\left(e_{\left(\left(\Sigma, j_{0}\right), P, J, \nu\right)} \times L \times M\right)^{-1}\left(\Delta_{T}^{0}\right)$ and $\left(e_{\left(\left(\Sigma, j_{1}\right), P, J, \nu\right)} \times L \times M\right)^{-1}\left(\Delta_{T}^{0}\right)$. This cobordism tells us that $\Phi_{\left(A_{1}, \cdots, A_{m}, \omega, \Sigma, P\right)}^{v}\left(\alpha_{1}, \cdots, \alpha_{c} \mid \beta_{1}, \cdots, \beta_{d}\right)(\gamma)$ is independent of the choice of the conformal structure $j$ on $\Sigma$.

(iii) We show that $\Phi_{\left(A_{1}, \cdots, A_{m}, \omega, \Sigma, P\right)}^{v}\left(\alpha_{1}, \cdots, \alpha_{c} \mid \beta_{1}, \cdots, \beta_{d}\right)(\gamma)$ is independent of the choice of the marked point set $X$ within the same position pattern $P_{1}$ (see the definition at the beginning of this section). If $X^{\prime}$ is another set of marked points, we choose a continuous map $\phi: \Sigma \rightarrow \Sigma$ isotropic to identity, such that it maps $X$ to $X^{\prime}$, and it is a diffeomorphic on each component $\Sigma_{i}, i=1, \cdots, m$. Let $\Sigma^{\prime}$ to be $\Sigma$ equipped with the pullback conformal structure $\phi^{*} j$, then obviously $\Phi_{\left(A_{1}, \cdots, A_{m}, \omega, \Sigma, P\right)}^{v}\left(\alpha_{1}, \cdots, \alpha_{c} \mid \beta_{1}, \cdots, \beta_{d}\right)(\gamma)$ defined using the stable curve $C^{\prime}=\left(\Sigma^{\prime}, X^{\prime}\right)$ is the same as the one defined using the stable curve $C=(\Sigma, X)$. Combining this with part ii) above, the independence of the choice $X$ is proved.

(iv) We show that $\Phi_{\left(A_{1}, \cdots, A_{m}, \omega, \Sigma, P\right)}^{v}\left(\alpha_{1}, \cdots, \alpha_{c} \mid \beta_{1}, \cdots, \beta_{d}\right)(\gamma)$ is independent of the choice of pseudo-manifold representatives $(F, Z),\left(L_{i}, U_{i}\right)$, $\left(M_{j}, W_{j}\right)$. Suppose that $\left(F_{0}, Z_{0}\right),\left(L_{i 0}, U_{i 0}\right),\left(M_{j 0}, W_{j 0}\right)$ and $\left(F_{1}, Z_{1}\right)$, $\left(L_{i 1}, U_{i 1}\right),\left(M_{j 1}, W_{j 1}\right)$ are two choices, both of them satisfying the general position condition. First we can find a cobordism of pseudomanifolds $\tilde{F}: \tilde{Z} \rightarrow Y$ with $\partial \tilde{F}=F_{0} \cup F_{1}, \partial \tilde{Z}=Z_{0} \cup Z_{1}$, and also construct a fiber bundle $\tilde{p}: \mathcal{X}_{\tilde{Z}} \rightarrow \tilde{Z}$ with fiber $V$ satisfying the Assumption $(\star)$. Secondly we can find a cobordism of pseudo-manifolds $\tilde{L}_{i}: \tilde{U}_{i} \rightarrow \mathcal{X}_{\tilde{Z}}, \tilde{M}_{j}: \tilde{W}_{j} \rightarrow \mathcal{X}_{\tilde{Z}}$, satisfying the pseudo-manifold representative condition listed at the beginning of this section (in particular the general position condition), and $\partial\left(\tilde{L}_{i}, \tilde{U}_{i}\right)=\left(L_{i 0}, U_{i 0}\right) \cup$ $\left(L_{i 1}, U_{i 1}\right), \partial\left(\tilde{M}_{j}, \tilde{W}_{j}\right)=\left(M_{j 0}, W_{j 0}\right) \cup\left(M_{j 1}, W_{j 1}\right)$. Apply the Remark 2.1 ii) to $(\tilde{F}, \tilde{Z}),\left(\tilde{L}_{i}, \tilde{U}_{i}\right),\left(M_{j}, \tilde{W}_{j}\right)$, we get a smooth one dimensional manifold which is a cobordism between $\left(e_{\left(\Sigma, P, J_{0}, \nu_{0}\right)} \times L_{0} \times M_{0}\right)^{-1}\left(\Delta_{T, 0}^{0}\right)$ and $\left(e_{\left(\Sigma, P, J_{1}, \nu_{1}\right)} \times L_{1} \times M_{1}\right)^{-1}\left(\Delta_{T, 1}^{1}\right)$ in the space $\mathcal{X}_{\tilde{Z}}$. This cobordism tells us that $\Phi_{\left(A_{1}, \cdots, A_{m}, \omega, \Sigma, P\right)}^{v}\left(\alpha_{1}, \cdots, \alpha_{c} \mid \beta_{1}, \cdots, \beta_{d}\right)(\gamma)$ is independent of the choice of $(F, Z),\left(L_{i}, U_{i}\right),\left(M_{j}, W_{j}\right)$. 
(v) Assume that two symplectic form $\omega_{0}$ and $\omega_{1}$ are connected to by a family of symplectic forms $\omega_{t}, t \in[0,1]$. Then an argument similar to (i) will prove that $\Phi_{\left(A_{1}, \cdots, A_{m}, \omega, \Sigma, P\right)}^{v}\left(\alpha_{1}, \cdots, \alpha_{c} \mid \beta_{1}, \cdots, \beta_{d}\right)(\gamma)$ depends only on the strong semi-positive deformation class of $\omega$, using fiber bundle $\mathcal{X} \times[0,1] \rightarrow Y \times[0,1]$ with fiberwise symplectic form $\left\{\omega_{t}: t \in\right.$ $[0,1]\}$.

Remark 2.2. To define

$$
\Phi_{\left(A_{1}, \cdots, A_{m}, \omega, \Sigma, P\right)}^{v}\left(\alpha_{1}, \cdots, \alpha_{c} \mid \beta_{1}, \cdots, \beta_{d}\right) \in H^{*}(Y, \mathbb{Q}),
$$

we only need to choose a basis $\gamma_{1}, \cdots, \gamma_{q}$ of $H^{*}(Y, \mathbb{Q})$, and evaluate $\Phi_{\left(A_{1}, \cdots, A_{m}, \omega, \Sigma, P\right)}^{v}\left(\alpha_{1}, \cdots, \alpha_{c} \mid \beta_{1}, \cdots, \beta_{d}\right)\left(\gamma_{i}\right)$. Note that for some appropriate choice of $\gamma_{1}, \cdots, \gamma_{q}$, we can represent all $\gamma_{i}$ by submanifolds $Z_{i}$ in $Y$. This means that we can choose $Z$ to be a manifold from the very beginning, and assume its cobordism to be smooth too. Had we proceeded in this way, our arguments could be much simplified. Actually in the definition of fiberwise quantum cohomology and equivariant quantum cohomology, $Z$ is always smooth. But there is a problem with this approach. When the homology groups with integer coefficient have torsion, we will have trouble in showing that (fiberwise) Gromov-Witten invariants is independent of different smooth manifold representations of some homology class, since these two smooth representation may not be cobordant to each other in smooth sense (see [C]). This is why we use pseudo-manifolds.

Next we collect a few simple properties of the fiberwise mixed invariants, leaving the composition law for the next section.

Proposition 2.3. The fiberwise mixed invariant

$$
\Phi_{\left(A_{1}, \cdots, A_{m}, \omega, \Sigma, P\right)}^{v}\left(\alpha_{1}, \cdots, \alpha_{c} \mid \beta_{1}, \cdots, \beta_{d}\right)(\gamma)
$$

is multilinear in $\alpha_{i}, \beta_{j}$ and $\gamma$. Furthermore, we have

(i) The fiberwise mixed invariant

$$
\Phi_{\left(A_{1}, \cdots, A_{m}, \omega, \Sigma, P\right)}^{v}\left(\alpha_{1}, \cdots, \alpha_{c} \mid \beta_{1}, \cdots, \beta_{d}\right)(\gamma)
$$

is zero if the "virtual" dimension $2 C_{1}(V) \cdot A+2 n(1-g)+\operatorname{deg} \gamma<0$.

(ii) $\Phi_{\left(A_{1}, \cdots, A_{m}, \omega, \Sigma, P\right)}^{v}\left(\alpha_{1}, \cdots, \alpha_{c} \mid \beta_{1}, \cdots, \beta_{d}\right)$ is zero if one of the $\beta_{j}$ is of degree greater than $2 n-2$. 
(iii) If $c+2 g \geq 4$ and $\alpha_{c}$ is the fundamental class $\mathcal{X}$, then

$$
\Phi_{\left(A_{1}, \cdots, A_{m}, \omega, \Sigma, P\right)}^{v}\left(\alpha_{1}, \cdots, \alpha_{c} \mid \beta_{1}, \cdots, \beta_{d}\right)(\gamma)
$$

equals

$$
\Phi_{\left(A_{1}, \cdots, A_{m}, \omega, \Sigma, P\right)}^{v}\left(\alpha_{1}, \cdots, \alpha_{c-1} \mid \beta_{1}, \cdots, \beta_{d}\right)(\gamma) .
$$

(iv) $\Phi_{\left(A_{1}, \cdots, A_{m}, \omega, \Sigma, P\right)}^{v}\left(\alpha_{1}, \cdots, \alpha_{c} \mid \beta_{1}, \cdots, \beta_{d}\right)(\gamma)$ equals

$$
D \cdot \Phi_{\left(A_{1}, \cdots, A_{m}, \omega, \Sigma, P\right)}^{v}\left(\alpha_{1}, \cdots, \alpha_{c} \mid \beta_{1}, \cdots, \beta_{d-1}\right)(\gamma),
$$

if $\beta_{d}$ is of degree 2 and $D=A \cdot \beta_{d}$ is the intersection number, where we regard $\beta_{d}$ as the induced class on $V$.

(v) In case $A=0, \Phi_{\left(A_{1}, \cdots, A_{m}, \omega, \Sigma, P\right)}^{v}\left(\alpha_{1}, \cdots, \alpha_{c} \mid \beta_{1}, \cdots, \beta_{d}\right)$ equals zero if $d>0$ and equals the intersection number $\int_{V} \alpha_{1} \cap \cdots \cap \alpha_{c}$ if $d=0$.

(vi) Let $\delta$ be a class in $H^{*}(Y, \mathbb{Q})$. Then $p^{*} \delta$ is a class in $H^{*}(\mathcal{X}, \mathbb{Q})$, and

$$
\begin{aligned}
& \Phi_{\left(A_{1}, \cdots, A_{m}, \omega, \Sigma, P\right)}^{v}\left(p^{*} \delta, \alpha_{1}, \cdots, \alpha_{c} \mid \beta_{1}, \cdots, \beta_{d}\right) \\
& =\delta \wedge \Phi_{\left(A_{1}, \cdots, A_{m}, \omega, \Sigma, P\right)}^{v}\left(\alpha_{1}, \cdots, \alpha_{c} \mid \beta_{1}, \cdots, \beta_{d}\right) .
\end{aligned}
$$

Proof. For (i), (ii), (iii), (iv), the proof, similar to the proof of Proposition 2.5 in [RT], is omitted.

(v) (2.1) implies $\sum_{1}^{c} \operatorname{deg} \alpha_{i}+\sum_{1}^{d}\left(\operatorname{deg} \beta_{j}-2\right)=2 n(1-g)+\operatorname{deg}(\gamma)$. Fix a pseudo-manifold representative $F: Z \rightarrow Y$, then the moduli space is $\mathcal{X}_{Z}$ if we choose the fiberwise inhomogeneous term to be zero. If we choose representatives $\left(L_{i}, U_{i}\right),\left(M_{j}, W_{j}\right)$ of $\alpha_{i}, \beta_{j}$ intersecting transversally in $\mathcal{X}_{Z}$, by definition $\Phi_{\left(A_{1}, \cdots, A_{m}, \omega, \Sigma, P\right)}^{v}\left(\alpha_{1}, \cdots, \alpha_{c} \mid \beta_{1}, \cdots, \beta_{d}\right)(\gamma)$ is the intersection number of $L_{1}\left(U_{1}\right) \cap \cdots \cap L_{c}\left(U_{c}\right) \cap M_{1}\left(W_{1}\right) \cap \cdots M_{d}\left(W_{d}\right)$. If $d>0$, this intersection is empty because of the degree requirement. If $d=0$, this intersection number is the same as

$$
\begin{aligned}
& \tilde{F}^{*} \alpha_{1} \wedge \cdots \wedge \tilde{F}^{*} \alpha_{c}\left(\mathcal{X}_{Z}\right) \\
& =\int_{V} \tilde{F}^{*} \alpha_{1} \wedge \cdots \wedge \tilde{F}^{*} \alpha_{c}(Z) \\
& =\int_{V} \alpha_{1} \wedge \cdots \alpha_{c}\left(F_{*} Z\right) \\
& =\int_{V} \alpha_{1} \wedge \cdots \alpha_{c}(\gamma)
\end{aligned}
$$


where $\tilde{F}: \mathcal{X}_{Z} \rightarrow \mathcal{X}$ is the natural map induced from $F: Z \rightarrow Y$. So (v) is proved.

(vi) Suppose that $F: Z \rightarrow Y$ is a pseudo-manifold representative of a class $\gamma \in H^{*}(Y, \mathbb{Q})$, then we have the diagram

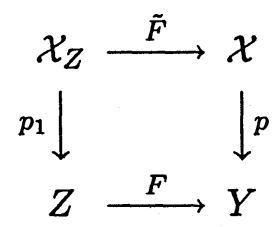

Note that since $\tilde{F}^{*} p^{*} \delta=p_{1}^{*} F^{*} \delta$, if the Poincare dual of $F^{*} \delta$ is represented by a pseudo-manifold $K$, then we can represent $\tilde{F}^{*} p^{*} \delta$ by the pseudomanifold $\left(L_{0}, p_{1}^{-1}(K)\right)$. So the intersection of $\mathcal{M}_{\left(A_{1}, \cdots, A_{m}\right)}^{v}\left(\Sigma, \mathcal{X}_{Z}, J, \nu\right)$ with $\left(L_{0}, p_{1}^{-1}(K)\right),\left(L_{i}, U_{i}\right),\left(M_{j}, W_{j}\right)$ in $\mathcal{X}_{Z^{0}}^{c+d+1}$ is the same as the intersection of $\mathcal{M}_{\left(A_{1}, \cdots, A_{m}\right)}^{v}\left(\Sigma, \mathcal{X}_{K}, J, \nu\right)$ with $\left(L_{i}, U_{i}\right),\left(M_{j}, W_{j}\right)$ in $\mathcal{X}_{K^{0}}^{c+d}$. This exactly means that $\Phi_{\left(A_{1}, \cdots, A_{m}, \omega, \Sigma, P\right)}^{v}\left(p^{*} \delta, \alpha_{1}, \cdots, \alpha_{c} \mid \beta_{1}, \cdots, \beta_{d}\right)(\delta . \gamma)=$ $\delta \wedge \Phi_{\left(A_{1}, \cdots, A_{m}, \omega, \Sigma, P\right)}^{v}\left(\alpha_{1}, \cdots, \alpha_{c} \mid \beta_{1}, \cdots, \beta_{d}\right)(\gamma)$. The proposition is proved.

Proposition 2.4 (Direct Product). Let $\mathcal{X}_{1} \rightarrow Y_{1}, \mathcal{X}_{2} \rightarrow Y_{2}$ be two fiber bundles with fibers $V_{1}, V_{2}$ and fiberwise symplectic forms $\omega_{1}, \omega_{2}$, respectively, and assume both satisfy the Assumption ( $\star$ ). Then $\mathcal{X}=\mathcal{X}_{1} \times \mathcal{X}_{2} \rightarrow Y_{1} \times Y_{2}$ is a fiber bundle with fiber $V_{1} \times V_{2}$ and fiberwise symplectic form $\omega=\omega_{1} \oplus \omega_{2}$, also satisfying the Assumption ( $*$ ), and

$$
\begin{aligned}
& \Phi_{\left(\left(A_{1}, A_{1}^{\prime}\right), \cdots,\left(A_{m}, A_{m}^{\prime}\right), \omega, \Sigma, P\right)}^{\mathcal{X}, v}\left(\alpha_{1} \otimes \alpha_{1}^{\prime}, \cdots, \alpha_{c} \otimes \alpha_{c}^{\prime} \mid \beta_{1} \otimes \beta_{1}^{\prime}, \cdots, \beta_{d} \otimes \beta_{d}^{\prime}\right)\left(\gamma \otimes \gamma^{\prime}\right) \\
& =\Phi_{\left(A_{1}, \cdots, A_{m}, \omega_{1}, \Sigma, P_{1}\right)}^{\mathcal{X}_{1}, v}\left(\alpha_{1}, \cdots, \alpha_{c} \mid \beta_{1}, \cdots, \beta_{d}\right)(\gamma) \\
& \quad \cdot \Phi_{\left(A_{1}^{\prime}, \cdots, A_{m}^{\prime}, \omega_{2}, \Sigma, P_{2}\right)}^{\mathcal{X}_{2}, v}\left(\alpha_{1}^{\prime}, \cdots, \alpha_{c}^{\prime} \mid \beta_{1}^{\prime}, \cdots, \beta_{d}^{\prime}\right)\left(\gamma^{\prime}\right),
\end{aligned}
$$

where $\Phi^{\mathcal{X}, v}, \Phi^{\mathcal{X}_{1}, v}$, and $\Phi^{\mathcal{X}_{2}, v}$ denote the fiberwise mixed invariants on $\mathcal{X}, \mathcal{X}_{1}$, and $\mathcal{X}_{2}$ respectively.

Proof. This follows directly from the definition of the fiberwise mixed invariants.

Proposition 2.5 (Restriction). Let $\mathcal{X} \rightarrow Y$ be a fiber bundle with fiber $V$ satisfying Assumption ( $\star$ ), and $h: Y_{1} \rightarrow Y$ be a smooth map. Denote by 
$\mathcal{X}_{Y_{1}}=\mathcal{X} \times_{f} Y_{1}$ to be the induced fiber bundle with fiber $V$ and the induced fiberwise symplectic form $\omega$. Then $\mathcal{X}_{Y_{1}} \rightarrow Y_{1}$ satisfies Assumption ( $\star$ ) and we have a natural map $H: \mathcal{X}_{Y_{1}} \rightarrow \mathcal{X}$. Furthermore

$$
\begin{aligned}
& \Phi_{\left(A_{1}, \cdots, A_{m}, \omega, \Sigma, P\right)}^{\mathcal{X}_{Y_{1}}, v}\left(H^{*} \alpha_{1}, \cdots, H^{*} \alpha_{c} \mid H^{*} \beta_{1}, \cdots, H^{*} \beta_{d}\right) \\
& =h^{*} \Phi_{\left(A_{1}, \cdots, A_{m}, \omega, \Sigma, P\right)}^{\mathcal{X}, v}\left(\alpha_{1}, \cdots, \alpha_{c} \mid \beta_{1}, \cdots, \beta_{d}\right) .
\end{aligned}
$$

Proof. We only need to show that for any $\gamma \in H^{*}\left(Y_{1}, \mathbb{Q}\right)$,

$$
\begin{aligned}
& \Phi_{\left(A_{1}, \cdots, A_{m}, \omega, \Sigma, P\right)}^{\mathcal{X}_{Y_{1}, v}}\left(H^{*} \alpha_{1}, \cdots, H^{*} \alpha_{c} \mid H^{*} \beta_{1}, \cdots, H^{*} \beta_{d}\right)(\gamma) \\
& =\Phi_{\left(A_{1}, \cdots, A_{m}, \omega, \Sigma, P\right)}^{\mathcal{X}, v}\left(\alpha_{1}, \cdots, \alpha_{c} \mid \beta_{1}, \cdots, \beta_{d}\right)\left(h_{*} \gamma\right)
\end{aligned}
$$

Choose a pseudo-manifold representative $(F, Z)$ of $\gamma$ in $Y_{1}$, and representatives $\left(L_{i}, U_{i}\right),\left(M_{j}, W_{j}\right)$ in $\mathcal{X}_{Z}$ of $H^{*} \alpha_{i}, H^{*} \beta_{j}$. Then the pseudo-manifold representative of $h_{*} \gamma$ is $(h \circ F, Z)$, and the pseudo-manifold representatives of $\alpha_{i}$ and $\beta_{j}$ in $\mathcal{X}_{Z}$ can be chosen to be $\left(L_{i}, U_{i}\right)$ and $\left(M_{j}, W_{j}\right)$, respectively. With these choices, both sides of the above equality amount to counting the number of intersections of $\mathcal{M}_{\left(A_{1}, \cdots, A_{m}\right)}^{v}\left(\Sigma, \mathcal{X}_{Z^{0}}, J, \nu\right)$ with $U^{0} \times W^{0}$ in $\mathcal{X}_{Z^{0}}^{c+d}$. Clearly they are the same. The proposition is proved.

Proposition 2.6 (Induction). Suppose that the fiber bundle $Y \rightarrow B$ has fiber $V_{2}$ with fiberwise symplectic form $\omega_{2}$ satisfying Assumption (*). Suppose also that the fiber bundle $\mathcal{X} \rightarrow Y$ has fiber $V_{1}$ with fiberwise symplectic form $\omega$ satisfying Assumption ( $\star$ ). Further assume that $\omega$ is also a fiberwise symplectic form satisfying Assumption $(\star)$ for the induced fiber bundle $\mathcal{X} \rightarrow B$ with fiber $V$. Let $A_{1}, \cdots, A_{m}$ be in $H_{2}\left(V_{1}, \mathbb{Z}\right)$. Then

$$
\begin{aligned}
& \Phi_{\left(A_{1}, \cdots, A_{m}, \omega, \Sigma, P\right)}^{\mathcal{X}, B, v}\left(\alpha_{1}, \cdots, \alpha_{c} \mid \beta_{1}, \cdots, \beta_{d}\right) \\
& =\int_{V_{2}} \Phi_{\left(A_{1}, \cdots, A_{m}, \omega, \Sigma, P\right)}^{\mathcal{X}, Y, v}\left(\alpha_{1}, \cdots, \alpha_{c} \mid \beta_{1}, \cdots, \beta_{d}\right)
\end{aligned}
$$

where $\Phi^{\mathcal{X}, B, v}$ and $\Phi^{\mathcal{X}, Y, v}$ are the fiberwise mixed invariants for the fiber bundle $\mathcal{X} \rightarrow B$ and $\mathcal{X} \rightarrow Y$ respectively, and the map $\int_{V_{2}}: H^{*}(Y, \mathbb{Q}) \rightarrow$ $H^{*-\operatorname{dim} V_{2}}(B, \mathbb{Q})$ is the Gysin map which can be defined by integration along the fiber direction.

Proof. For any $\gamma \in H_{*}(B, \mathbb{Q})$, choose its pseudo-manifold representative $F: Z \rightarrow B$. Denote $Y \times{ }_{F} Z$ by $\tilde{Z}$. Then $\tilde{F}: \tilde{Z} \rightarrow Y$ is a pseudo-manifold 
representing a homology class $\tilde{\gamma} \in H_{*}(Y, \mathbb{Q})$. All we need to show is that

$$
\begin{aligned}
& \Phi_{\left(A_{1}, \cdots, A_{m}, \omega, \Sigma, P\right)}^{\mathcal{X}, B, v}\left(\alpha_{1}, \cdots, \alpha_{c} \mid \beta_{1}, \cdots, \beta_{d}\right)(\gamma) \\
& =\Phi_{\left(A_{1}, \cdots, A_{m}, \omega, \Sigma, P\right)}^{\mathcal{X}, Y, v}\left(\alpha_{1}, \cdots, \alpha_{c} \mid \beta_{1}, \cdots, \beta_{d}\right)(\tilde{\gamma}) .
\end{aligned}
$$

But $\mathcal{X}_{Z} \rightarrow Z$ is a bundle with fiber $V$, and $\mathcal{X}_{\tilde{Z}} \rightarrow \tilde{Z}$ is a bundle with fiber $V_{1}$. Obviously there is a natural isomorphism $\mathcal{X}_{Z} \cong \mathcal{X}_{\tilde{Z}}$, so we can choose the pseudo-manifolds representing $\alpha_{i}, \beta_{j}$ in $\mathcal{X}_{Z}$ and $\mathcal{X}_{\tilde{Z}}$ to be the same. To prove above identity, we need to find a good $(J, \nu)$ for $\mathcal{X}_{Z}$ and a $\operatorname{good}(\tilde{J}, \tilde{\nu})$ for $\mathcal{X}_{\tilde{Z}}$ such that $\mathcal{M}_{\left(A_{1}, \cdots, A_{m}\right)}\left(\Sigma, \mathcal{X}_{Z}, J, \nu\right)$ is naturally identified with $\mathcal{M}_{\left(A_{1}, \cdots, A_{m}\right)}\left(\Sigma, \mathcal{X}_{\tilde{Z}}, \tilde{J}, \tilde{\nu}\right)$ and they define the same evaluation map.

We achieve this goal in two steps. In step 1, we will show that there are a family of $(J, \nu)$ for $\mathcal{X}_{Z} \rightarrow Z$ such that any fiberwise $(J, \nu)$ holomorphic map actually lies in the fiber $V_{1}$ of the fiber bundle $V \rightarrow V_{2}$, i.e., it is actually a fiberwise $(\tilde{J}, \tilde{\nu})$ holomorphic map for $\mathcal{X}_{\tilde{Z}}$. So we have an isomorphism between $\mathcal{M}_{\left(A_{1}, \cdots, A_{m}\right)}\left(\Sigma, \mathcal{X}_{Z}, J, \nu\right)$ and $\mathcal{M}_{\left(A_{1}, \cdots, A_{m}\right)}\left(\Sigma, \mathcal{X}_{\tilde{Z}}, \tilde{J}, \tilde{\nu}\right)$. In step 2, we will show that there are good $(J, \nu)$ and $(\tilde{J}, \tilde{\nu})$ among the above mentioned family.

Step 1. Let $E, E_{1}$, and $E_{2}$ be vector bundle over $\mathcal{X}_{Z}, \mathcal{X}_{\tilde{Z}}$, and $\tilde{Z}$ respectively as defined after Remark 1.2. Note that $E_{1}$ is a subbundle of $E$. Fix a splitting $E=E_{1} \oplus E_{1}^{c}$, then we have diagram with $\pi_{1 *}$ being an isomorphism on the fiber.

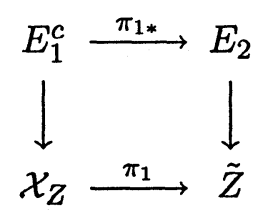

We define a family of $(J, \nu)$ on $E$ as follows. They are of the form $\left.(\tilde{J}, \tilde{\nu})\right|_{E_{1}} \oplus$ $\left.\left(J_{2}, 0\right)\right|_{E_{1}^{c}}$, where $(\tilde{J}, \tilde{\nu}) \in \mathcal{P}_{k-1, p}\left(E_{1}, \omega\right)$ and $J_{2}$ is the pull-back of fiberwise almost complex structure $\tilde{J}_{2} \in \mathcal{P}_{k-1, p}\left(E_{2}, \omega_{2}\right)$ by $\pi_{1 *}$. Note that $J$ is tamed by $\omega+\epsilon \pi_{1}^{*} \omega_{2}$ for small $\epsilon$. We show now that for such $(J, \nu)$, if $f$ is a fiberwise $(J, \nu)$ holomorphic curve whose image is in fiber $V$, then $\pi_{1} \circ f=$ constant where $V \stackrel{\pi_{1}}{\longrightarrow} V_{2}$ is a bundle map, i.e., it is in $\mathcal{M}_{\left(A_{1}, \cdots, A_{m}\right)}^{v}\left(\Sigma, \mathcal{X}_{\tilde{Z}}, \tilde{J}, \tilde{\nu}\right)$. By definition, $d f+J \circ d f \circ j_{\Sigma}=(\tilde{\nu}, 0)$. Projecting it to $E_{2}$, we get $\pi_{1 *} d f+\pi_{1 *} J \circ$ $d f \circ j_{\Sigma}=\pi_{1 *}(\tilde{\nu}, 0)$. Since $\pi_{1 *} J=J_{2} \pi_{1 *}$, we get $d\left(\pi_{1} \circ f\right)+J_{2} \circ d\left(\pi_{1} \circ f\right) \circ j_{\Sigma}=0$, i.e., $\pi_{1} \circ f$ is a $J_{2}$ holomorphic map in $V_{2}$. But since $A \in H_{2}\left(V_{1}, \mathbb{Z}\right)$,

$$
0=\int_{A} \pi_{1}^{*} \omega_{2}=\int_{f(\Sigma)} \pi_{1}^{*} \omega_{2}=\int_{\Sigma} f^{*} \pi_{1}^{*} \omega_{2}=\int_{\Sigma}\left(\pi_{1} \circ f\right)^{*} \omega_{2}
$$


on the other hand $\int_{\Sigma}\left(\pi_{1} \circ f\right)^{*} \omega_{2}>0$ if $\pi_{1} \circ f$ is not a constant.

Step 2. The existence of a good $(J, \nu)$ can be proved in the following way. We can choose a subspace of $\operatorname{Map}_{\left(A_{1}, \cdots, A_{m}\right)}(\Sigma, \mathcal{X})$ consisting of maps with $f(\Sigma) \subset V_{1} \times p t$, and choose a subspace of $\mathcal{P}_{k-1, p}^{v}(\mathcal{X}, \omega)$ consisting of all $(J, \nu)$ described above. Now the argument of Proposition 1.4 works within these two subspaces.

The proposition is proved.

Remark 2.3. Assume that $(\mathcal{X}, \omega)$ itself is a symplectic manifold, and $\omega$ is also a fiberwise symplectic form for a bundle $\mathcal{X} \rightarrow Y$. Note that $\Phi_{\left(A_{1}, \cdots, A_{m}, \omega, \Sigma, P\right)}^{\mathcal{X}, p t,}\left(\alpha_{1}, \cdots, \alpha_{c} \mid \beta_{1}, \cdots, \beta_{d}\right)$ is equal to the non-fiberwise mixed invariant $\Phi_{\left(A_{1}, \cdots, A_{m}, \omega, \Sigma, P\right)}^{v}\left(\alpha_{1}, \cdots, \alpha_{c} \mid \beta_{1}, \cdots, \beta_{d}\right)$ if $A_{1}, \cdots, A_{m} \in H_{2}(V ; \mathbb{Z})$. Choosing $B=p t$ in the above proposition, the non-fiberwise mixed invariant and the fiberwise mixed invariant are related by

$$
\begin{aligned}
& \int_{Y} \Phi_{\left(A_{1}, \cdots, A_{m}, \omega, \Sigma, P\right)}^{\mathcal{X}, Y, v}\left(\alpha_{1}, \cdots, \alpha_{c} \mid \beta_{1}, \cdots, \beta_{d}\right) \\
& =\Phi_{\left(A_{1}, \cdots, A_{m}, \omega, \Sigma, P\right)}^{v}\left(\alpha_{1}, \cdots, \alpha_{c} \mid \beta_{1}, \cdots, \beta_{d}\right) .
\end{aligned}
$$

In this circumstance, using the above identity, all the properties including the composition law of $\Phi_{\left(A_{1}, \cdots, A_{m}, \omega, \Sigma, P\right)}^{\mathcal{X}, Y, v}\left(\alpha_{1}, \cdots, \alpha_{c} \mid \beta_{1}, \cdots, \beta_{d}\right)$ follow from the corresponding properties of the non-fiberwise mixed invariants. This fact was already observed by Astashkevich and Sadov in [AS].

\section{Composition Law of Fiberwise Gromov-Witten Mixed Invariants.}

\subsection{Gluing of $J$-Holomorphic Maps.}

In this subsection, we will apply the implicit function theorem to the study of deformation theory of perturbed $J$-holomorphic maps from a singular curve.

Recall that a degeneration of stable curves is a holomorphic fibration $\pi: S \rightarrow \Delta \subset \mathbb{C}$ with sections $\sigma_{1}, \cdots, \sigma_{c}$ satisfying : (1) For each $t \in \Delta, t \neq 0$, the fiber $\Sigma_{t}=\pi^{-1}(t)$ is smooth; (2) For each $t, C_{t}=\left(\Sigma_{t} ; \sigma_{1}(t), \cdots, \sigma_{c}(t)\right)$ is a $c$-point stable curve.

Adopting the notation of section 1, suppose that $\mathcal{X} \stackrel{p}{\rightarrow} Y$ is a fiber bundle with fiber $V$ satisfying Assumption ( $\star$ ) $, F: Z \rightarrow Y$ is a pseudo- 
manifold, and $Z^{\alpha}$ is a stratum in $Z$. For any point $z_{0} \in Z^{\alpha}$, we can find a neighborhood $W_{\alpha} \subset Z^{\alpha}$ of $z_{0}$ such that there is a trivialization $\left.\mathcal{X}_{Z}\right|_{W^{\alpha}} \cong$ $W^{\alpha} \times V$. Denoting $\left.\mathcal{X}_{Z}\right|_{W^{\alpha}}$ by $\mathcal{X}_{W^{\alpha}}$, we equip $W^{\alpha}$ with Euclidean metric $d s_{0}^{2}$ and trivial connection $\nabla_{1}$. Let $h_{E}$ be a Hermitian metric on bundle $E, \nabla$ be the connection on $E$ compatible with $h_{E}$ and $J$, with torsion $\frac{1}{4} N_{J} ; N_{J}$ is again the Nijenhuis tensor. Therefore $h_{\alpha}=d s_{0}^{2}+h_{E}$ is a metric on $W^{\alpha} \times V$.

Let $\nu$ be an inhomogeneous term on $S \times \mathcal{X}_{Z}$, and $\nu_{t}$ be the restriction of $\nu$ to $\Sigma_{t} \times \mathcal{X}_{Z}$. Consider the moduli space of fiberwise $\left(J, \nu_{t}\right)$-perturbed holomorphic maps in $\mathcal{X}_{Z^{\alpha}}$,

$$
\begin{aligned}
& \mathcal{M}_{A}^{v}\left(\Sigma_{t}, \mathcal{X}_{Z^{\alpha}}, J, \nu_{t}\right) \\
& =\left\{f: \Sigma_{t} \rightarrow \mathcal{X}_{Z^{\alpha}} \mid d f+J \circ d f \circ j_{t}=\nu_{t},\left[f\left(\Sigma_{t}\right)\right]=A, p \circ f\left(\Sigma_{t}\right)=p t \in Z^{\alpha}\right\},
\end{aligned}
$$

where $j_{t}$ is the complex structure on $\Sigma_{t}(t \neq 0), A$ is a fixed homology class in $H_{2}(V, \mathbb{Z})$.

Assume that $\Sigma_{0}$ has m-components $\Sigma_{01}, \cdots, \Sigma_{0 m}$, and suppose that the position pattern of $\sigma_{1}(0), \cdots, \sigma_{c}(0)$ in $\Sigma_{0}$ is $P_{1}$. Fix a partition of

$$
A=A_{1}+\cdots A_{m}, \quad A_{i} \in H_{2}(V ; \mathbb{Z}),
$$

which is effective, i.e., $\mathcal{M}_{A_{i}}^{v}\left(\Sigma_{0 i}, \mathcal{X}_{Z^{\alpha}}, J, \nu_{0 i}\right)$ is not empty for each $i$. From Proposition 1.2, the moduli space $\mathcal{M}_{\left(A_{1}, \cdots, A_{m}\right)}^{v}\left(\Sigma_{0}, \mathcal{X}_{Z^{\alpha}}, J, \nu_{0}\right)$ is a smooth manifold and for generic $t \neq 0, \mathcal{M}_{A}^{v}\left(\Sigma_{t}, \mathcal{X}_{Z^{\alpha}}, J, \nu_{t}\right)$ is also smooth manifold with dimension

$$
\operatorname{dim} \mathcal{M}_{A}^{v}\left(\Sigma_{t}, \mathcal{X}_{Z^{\alpha}}, J, \nu_{t}\right)=2 C_{1}(V) \cdot A+2 n(1-g)+\operatorname{dim} Z^{\alpha},
$$

where $2 n$ is the real dimension of $V$ and $g$ is the genus of $\Sigma_{t}$.

Theorem 3.1. Let $f_{0}$ be any map in $\mathcal{M}_{\left(A_{1}, \cdots, A_{m}\right)}^{v}\left(\Sigma_{0}, \mathcal{X}_{Z^{\alpha}}, J, \nu_{0}\right)$ which intersects transversally at each double point. Assume $f_{0}\left(\Sigma_{0}\right) \subset V_{z_{0}}$. Then there is a continuous family of injective maps $T_{t}$ from $\tilde{W}$ into $\mathcal{M}_{A}^{v}\left(\Sigma_{t}, \mathcal{X}_{Z^{\alpha}}, J, \nu_{t}\right)$, where $t$ is small and $\tilde{W}$ is a neighborhood of $f_{0}$ in $\mathcal{M}_{\left(A_{1}, \cdots, A_{m}\right)}^{v}\left(\Sigma_{0}, \mathcal{X}_{W^{\alpha}}, J, \nu_{0}\right)$ such that:

(1) for any $f$ in $\tilde{W}$, as $t$ goes to zero, $T_{t}(f)$ converges to $f$ in $C^{0}$-topology on $\Sigma_{0}$ and in $C^{3}$-topology outside the singular set of $\Sigma_{0}$;

(2) there are $\epsilon, \delta>0$ satisfying: if $f^{\prime}$ is in $\mathcal{M}_{A}^{v}\left(\Sigma_{t}, \mathcal{X}_{W^{\alpha}}, J, \nu_{t}\right)$ and $d_{\mathcal{X}_{W^{\alpha}}}\left(f^{\prime}(x), f_{0}(y)\right) \leq \epsilon$ whenever $x \in \Sigma_{t}, y \in \Sigma_{0}, d_{S}(x, y) \leq \delta$, where $d_{\mathcal{X}_{W^{\alpha}}}$ and $d_{S}$ are the distance functions of metric $h_{\alpha}$ on $\mathcal{X}_{W^{\alpha}}$ and a Kähler metric $\mu$ on $S$, respectively, then $f^{\prime}$ is in $T_{t}(\tilde{W})$. Moreover, for generic $t$, $T_{t}$ is an orientation preserving smooth map from $\tilde{W}$ into smooth manifold $\mathcal{M}_{A}^{v}\left(\Sigma_{t}, \mathcal{X}_{Z^{\alpha}}, J, \nu_{t}\right)$. 
Proof. First, we make a reduction to the case with zero fiberwise inhomogeneous term as we did in subsection 1.1. Adopting the notation there, for any deformation of $f_{0}, f_{t}: \Sigma_{t} \rightarrow \mathcal{X}_{Z^{\alpha}}$, its graph map $F_{t}: \Sigma_{t} \rightarrow S \times \mathcal{X}_{Z^{\alpha}}$ is a deformation of the graph map of $f_{0} . F_{0}: \Sigma_{0} \rightarrow S \times \mathcal{X}_{Z^{\alpha}}$. Let $\pi_{i}$ be the projection map from $S \times \mathcal{X}_{Z^{\alpha}}$ onto the i-th factor (i=1,2), then $\pi_{1} \circ F_{t}=i d$. Conversely if $\tilde{F}_{t}$ is a small deformation of $F_{0}$, then $\pi_{1} \circ \tilde{F}_{t}$ is indeed a biholomorphism of $\Sigma_{t}$, and $\tilde{f}_{t}=\pi_{2} \circ \tilde{F}_{t} \circ\left(\pi_{1} \circ \tilde{F}_{t}\right)^{-1}$ is a $\left(J, \nu_{t}\right)$-perturbed holomorphic map, a deformation of $f_{0}$. This shows that to study the deformation of $f_{0}$ is equivalent to study the deformation of $F_{0}$. So we may assume $\nu=0$. Note that although $S \times \mathcal{X}_{Z^{\alpha}}$ may not be compact, the object under study lies in a compact region.

In the following proof, we will use $C$ to denote a constant independent of $t$ and any $f$ near $f_{0}$. The actual value of $C$ may vary in different places.

Let $f \in \tilde{W}, f\left(\Sigma_{0}\right) \subset V_{z}$, and $z \in W^{\alpha}$. We will first construct an approximate $J$-holomorphic map from $\Sigma_{t}$ into $V_{z}$ for each $t$. Let $p$ be any double point of $\Sigma_{0}$, and $U_{p}$ be a small neighborhood in $S$ containing $p . U_{p}$ has coordinate $\left(z_{p 1}, z_{p 2}\right)$ such that

$$
U_{p} \cap \Sigma_{t}=\left\{\left(z_{p 1}, z_{p 2}\right)\left|z_{p 1} \cdot z_{p 2}=t,\right| z_{p 1}|<1,| z_{p 2} \mid<1\right\} .
$$

Choose a coordinate system $u_{1}, \cdots, u_{\tilde{n}} ; y_{1}, \cdots, y_{2 n}$ of $\mathcal{X}_{W^{\alpha}}$ near $f(p)$ $(\tilde{n}=\operatorname{dim} W)$, such that the coordinate of $f(p)$ is zero and

$$
\begin{aligned}
& J\left(\frac{\partial}{\partial y_{i}}\right)=\frac{\partial}{\partial y_{n+i}}+O(|y|+|u|), \\
& J\left(\frac{\partial}{\partial y_{n+i}}\right)=-\frac{\partial}{\partial y_{i}}+O(|y|+|u|),
\end{aligned}
$$

where $i=1, \cdots, n,|y|=\sqrt{\sum_{i=1}^{2 n}\left|y_{i}\right|^{2}}$, and $|u|=\sqrt{\sum_{i=1}^{\tilde{n}}\left|u_{i}\right|^{2}}$. Note that there are two connected components in $U_{p} \cap \Sigma_{0}$,

$$
U_{p 1}=\left\{\left(z_{p 1}, 0\right)|| z_{p 1} \mid<1\right\} \text { and } U_{p 2}=\left\{\left(0, z_{p 2}\right)|| z_{p 2} \mid<1\right\} .
$$

Let $f_{p i}=\left.f\right|_{U_{p i}}, i=1,2$. Then we have the following expansions:

$$
f_{p i}\left(z_{p i}\right)=\tilde{f}_{p i}\left(z_{p i}\right)+\text { terms of degree greater than } 1,
$$

where $\tilde{f}_{p i}$ are homogeneous polynomials in $z_{p i}$ of degree 1 . We identify a neighborhood of $f(p)$ in $\mathcal{X}_{W^{\alpha}}$ with open set in $W^{\alpha} \times \mathbb{C}^{n}$ by putting $w_{i}=$ $y_{i}+\sqrt{-1} y_{n+i}, i=1, \cdots, n$. By assumption, at each double point, $\left.f_{0}\right|_{U_{p 1}}$ and 
$\left.f_{0}\right|_{U_{p 2}}$ intersect transversally. If we choose $\tilde{W}$ small enough, we may assume that for any $\left.f \in \tilde{W} f\right|_{U_{p 1}}$ and $\left.f\right|_{U_{p 2}}$ also intersect transversally. Then by choosing $y_{1}, \cdots, y_{2 n}$ properly, we have

$$
\begin{array}{ll}
f_{p 1}\left(\left(z_{p 1}, 0\right)\right)=\left(z_{p 1}, 0, \cdots, 0\right)+O\left(\left|z_{p 1}\right|^{2}\right) & \in 0 \times \mathbb{C}^{n}, \\
f_{p 2}\left(\left(0, z_{p 2}\right)\right)=\left(0, z_{p 2}, \cdots, 0\right)+O\left(\left|z_{p 2}\right|^{2}\right) & \in 0 \times \mathbb{C}^{n} .
\end{array}
$$

By changing local coordinates $y_{1}, \cdots, y_{2 n}$, we may further assume that

$$
\begin{aligned}
& f_{p 1}\left(\left(z_{p 1}, 0\right)\right)=\left(z_{p 1}, 0, \cdots, 0\right), \\
& f_{p 2}\left(\left(0, z_{p 1}\right)\right)=\left(0, z_{p 2}, \cdots, 0\right) .
\end{aligned}
$$

Now we begin to construct an approximate $J$-holomorphic map $f_{t}^{a p p}$ : $\Sigma_{t} \rightarrow V_{z}$ for each small $t$. Let $\phi_{t}$ be a smooth family of diffeomorphism from $\Sigma_{0}^{\prime}$ into $\Sigma_{t}$, where $\Sigma_{0}^{\prime}$ is the nonsingular part of $\Sigma_{0}$, such that $\phi_{0}=i d$, and

$$
\left\|\phi_{t}-i d\right\|_{C^{5}\left(\Sigma_{0} \backslash U^{\prime}\right)} \leq C_{U^{\prime}} \cdot t
$$

for any small neighborhood $U^{\prime}$ of the singular set $\operatorname{Sing}\left(\Sigma_{0}\right)$ in $\Sigma_{0}$. Here $C_{U^{\prime}}$ is a constant depending only on $U^{\prime}$, and the norm is taken with respect to metric $\mu$ on $S$ by viewing both $\phi_{t}$ and $i d$ as maps into $S$. Then for any $p$ in $\operatorname{Sing}\left(\Sigma_{0}\right)$, we have

$$
\left.|| \tilde{f}\right|_{\Sigma_{t} \cap U_{p}}-f \circ \phi_{t}^{-1} \|_{C^{4}\left(\Sigma_{t} \cap\left\{\left(z_{p 1}, z_{p 2}\right)\left|\frac{1}{2} \leq\right| z_{p i} \mid \leq 1, i=1 \text { or } 2\right\}\right)} \leq C \cdot|t|,
$$

where $\tilde{f}$ is the map: $\left(z_{p 1}, z_{p 2}\right) \in U_{p} \rightarrow\left(z_{p 1}, z_{p 2}, 0, \cdots, 0\right) \in 0 \times \mathbb{C}^{n}$. Note that

$$
\left.\tilde{f}\right|_{U_{p 1}}=f_{p 1},\left.\quad \tilde{f}\right|_{U_{p 2}}=f_{p 2} .
$$

By (3.4),(3.5), there is a homotopy $F_{t}$ on $\Sigma_{t} \cap\left(\cup_{p \in \operatorname{Sing}\left(\Sigma_{0}\right)}\left\{\left(z_{p 1}, z_{p 2}\right) \mid \frac{1}{2} \leq\right.\right.$ $\left|z_{p i}\right| \leq 1, i=1$ or 2$\left.\}\right)$ satisfying, for any $p \in \operatorname{Sing}\left(\Sigma_{0}\right)$

$$
\left\|F_{t}-f \circ \phi_{t}^{-1}\right\|_{C^{4}\left(\Sigma_{t} \cap\left\{\left(z_{p 1}, z_{p 2}\right)\left|\frac{1}{2} \leq\right| z_{p i} \mid \leq 1, i=1 \text { or } 2\right\}\right)} \leq C \cdot|t|
$$

$$
F_{t}=\tilde{f} \text { on } \Sigma_{t} \cap\left\{\left(z_{p 1}, z_{p 2}\right)\left|\frac{1}{2} \leq\right| z_{p i} \mid \leq \frac{7}{10}, i=1 \text { or } 2\right\}
$$

$$
F_{t}=f \circ \phi_{t}^{-1} \text { on } \Sigma_{t} \cap\left\{\left(z_{p 1}, z_{p 2}\right)\left|\frac{9}{10} \leq\right| z_{p i} \mid \leq 1, i=1 \text { or } 2\right\} .
$$


We define

$$
\begin{aligned}
& f_{t}^{a p p}(x)=f \circ \phi_{t}^{-1}(x), \quad \text { for } x \in \Sigma_{t} \backslash \cup_{p \in \operatorname{Sing}\left(\Sigma_{0}\right)} U_{p} \\
& f_{t}^{a p p}\left(z_{p 1}, z_{p 2}\right)=F_{t}\left(z_{p 1}, z_{p 2}\right), \quad \text { if } \frac{1}{2} \leq\left|z_{p i}\right| \leq 1, i=1 \text { or } 2, \\
& f_{t}^{a p p}\left(z_{p 1}, z_{p 2}\right)=\left(z_{p 1}, z_{p 2}, 0, \cdots, 0\right), \quad \text { if }\left|z_{p i}\right| \leq \frac{1}{2}, i=1 \text { and } 2 .
\end{aligned}
$$

Let $\mu$ be a Kähler metric on $S$ which equals $d z_{p 1} \otimes d \bar{z}_{p 1}+d z_{p 2} \otimes d \bar{z}_{p 2}$ on $U_{p}$ for each $p \in \operatorname{Sing}\left(\Sigma_{0}\right)$. Let $\rho$ be a smooth function on $S \backslash \operatorname{Sing}\left(\Sigma_{0}\right)$ satisfying $0<\rho \leq 3$ and

$$
\left.\rho\right|_{U_{p}}\left(z_{p 1}, z_{p 2}\right)=\sqrt{\left|z_{p 1}\right|^{2}+\left|z_{p 2}\right|^{2}}, \quad \text { for each } p \in \operatorname{Sing}\left(\Sigma_{0}\right)
$$

Define a new metric $\mu_{c}$ on $S, \mu_{c}=\rho^{-2} \mu$. Note that

$$
\mu_{c} \mid \Sigma_{t} \cap U_{p}=\frac{\left|d z_{p i}\right|^{2}}{\left|z_{p i}\right|^{2}}
$$

i.e., for $t$ small, $\mu_{c} \mid \Sigma_{t} \cap U_{p}$ is a cylinderlike metric. The following lemma can be easily proved from the definition of $f_{t}^{a p p}, \mu$, and $\mu_{c}$ by local computation.

Lemma 3.1. Let $\nabla$ be the connection defined at the beginning of this section. Denote by $D$ the covariant derivative induced from $\mu$ and $\nabla$, and $D_{c}$ the covariant derivative induced from $\mu_{c}$ and $\nabla$. Then for $1 \leq k \leq 5$,

$$
\begin{aligned}
& \left|D^{k} f_{t}^{a p p}\right|_{\mu, h_{E}}(x) \leq C_{k}\left(1+\frac{|t|}{\rho(x)^{k+1}}\right) \\
& \left|D_{c}^{k} f_{t}^{a p p}\right|_{\mu_{c}, h_{E}}(x) \leq C_{k}^{\prime}\left(1+\frac{|t|}{\rho(x)}\right)
\end{aligned}
$$

where ||$_{\mu, h_{E}}$ and ||$_{\mu_{c}, h_{E}}$ denote the norm defined by $\mu, h_{E}$ and $\mu_{c}, h_{E}$ respectively.

Let $J_{0}$ be the standard complex structure on $\mathbb{C}^{n}$. By definition, for each $p$ in $\operatorname{Sing}\left(\Sigma_{0}\right)$

$$
d f_{t}^{a p p}+J_{0} \circ d f_{t}^{a p p} \circ j_{t}=0 \quad \text { on } \Sigma_{t} \cap\left\{\left(z_{p 1}, z_{p 2}\right)|| z_{p i} \mid \leq \frac{1}{2}, i=1 \text { and } 2\right\}
$$

where $j_{t}=j_{\Sigma_{t}}$. Put

$$
v_{t}(x)=\left(d f_{t}^{a p p}+J \circ d f_{t}^{a p p} \circ j_{t}\right)(x) .
$$


Then

$$
v_{t}(x)=\left(J-J_{0}\right) \circ d f_{t}^{a p p} \circ j_{t}(x)
$$

on

$$
\Sigma_{t} \cap\left\{\left(z_{p 1}, z_{p 2}\right)|| z_{p i} \mid \leq \frac{1}{2}, i=1 \text { and } 2\right\} .
$$

Since $f$ is $J$-holomorphic on $\Sigma_{0} \cap\left\{\left(z_{p 1}, z_{p 2}\right)|| z_{p i} \mid \leq \frac{1}{2}, i=1\right.$ and 2$\}$, we have

$$
\begin{aligned}
& J\left(z_{p 1}, 0, \cdots, 0\right)\left(f_{*} \frac{\partial}{\partial z_{p 1}}\right)=J_{0}\left(z_{p 1}, 0, \cdots, 0\right)\left(f_{*} \frac{\partial}{\partial z_{p 1}}\right) \\
& J\left(0, z_{p 2}, \cdots, 0\right)\left(f_{*} \frac{\partial}{\partial z_{p 2}}\right)=J_{0}\left(0, z_{p 2}, \cdots, 0\right)\left(f_{*} \frac{\partial}{\partial z_{p 2}}\right) .
\end{aligned}
$$

Then we can derive from last lemma,

Lemma 3.2. For $1 \leq k \leq 4$, then

$$
\begin{aligned}
& \left|D^{k} v_{t}\right|_{\mu, h_{E}}(x) \leq C_{k} \cdot \frac{|t|}{\rho(x)^{k+1}}, \\
& \left|D_{c}^{k} v_{t}\right|_{\mu_{c}, h_{E}}(x) \leq C_{k}^{\prime} \cdot|t| .
\end{aligned}
$$

We want to perturb $f_{t}^{a p p}$ into a $J$-holomorphic map from $\Sigma_{t}$ into $\mathcal{X}_{W^{\alpha}}$. Fixing the trivialization $\mathcal{X}_{W^{\alpha}} \cong W^{\alpha} \times V$ as before, we can represent $f_{t}^{a p p}$ as $\left(z, f_{t 1}^{a p p}\right)$. Identifying $W^{\alpha}$ with an open subset of $\mathbb{R}^{\tilde{n}}, z$ with 0 and $W^{\alpha}$ with a neighborhood with $T_{z} W^{\alpha}$, we can define a modified exponential map

$$
\begin{gathered}
\exp ^{*}: T_{z} W^{\alpha} \times \Gamma\left(\Sigma_{t}, f_{t}^{a p p, *} T(z \times V)\right) \rightarrow W^{\alpha} \times V, \\
\left(u_{t 1}, u_{t 2}\right) \rightarrow\left(u_{t 1}, \exp _{f_{t}^{a p p}}^{*}\left(u_{t 1}, u_{t 2}\right)\right),
\end{gathered}
$$

as follows. Suppose $v^{i}$ is coordinate of $W^{\alpha}, y^{j}$ is coordinate around $f_{t 1}^{a p p}(x)$ in $V, x \in \Sigma_{t}$. Then $\exp _{f_{t}^{a p p}(x)}^{*}\left(u_{t 1}, u_{t 2}\left(f_{t}^{a p p}(x)\right)\right)$ is defined to be $y(1)$, where $y(s)$ is the solution of

$$
\left\{\begin{array}{l}
\frac{d^{2} y^{j}}{d s^{2}}+\Gamma_{k l}^{j}\left(s u_{t 1}, y\right) \frac{d y^{k}}{d s} \frac{d y^{l}}{d s}=0 \\
y(0)=f_{t 1}^{a p p}(x) \\
\left.\frac{d y}{d s}\right|_{s=0}=u_{t 2}\left(f_{t 1}^{a p p}(x)\right)
\end{array}\right.
$$


Here $\Gamma_{k l}^{j}\left(s u_{t 1}, y\right)$ is the Christoffel symbol of connection $\nabla$ on $E$. By definition the image of map exp* lies in the fiber $u_{t 1} \times V$. We do not use exponential map defined by metric $h_{\alpha}$, because its image does not lie in a fiber. Note that when we choose $u_{t 1}=0, e x p^{*}$ is exponential map on $z \times V$, defined by metric $\left.h_{E}\right|_{z \times V}$. So by the implicit function theorem and the property that solution of ordinary differential equation depends smoothly on parameters, we get that for small $u_{t 1}, \exp _{f_{t}^{a p p}(x)}^{*}$ is a diffeomorphism on a small ball in $f_{t}^{a p p, *} T_{f_{t}^{a p p}(x)}(z \times V)$. Let $f_{t}$ be a map from $\Sigma_{t}$ into $W^{\alpha} \times V$, we can represent it as $\left(z_{t}, f_{t 1}\right)$. If $f_{t}$ is sufficiently close to $f_{t}^{a p p}=\left(z, f_{t 1}^{a p p}\right)$, then we can write

$$
\left(z_{t}, f_{t 1}(x)\right)=\left(u_{t 1}, \exp _{f_{t}^{a p p}(x)}^{*}\left(u_{t 1}, u_{t 2}\left(f_{t 1}^{a p p}(x)\right)\right)\right),
$$

where $u_{t 1}$ is a vector in $T_{z} W^{\alpha}$ and $u_{t 2}$ is a vector field of $f_{t}^{a p p, *} T(z \times V)$ on $\Sigma_{t}$. We need to find $\left(u_{t 1}, u_{t 2}\right)$ such that $f_{t}$ is $J$-holomorphic.

For any $\left(u_{t 1}, u_{t 2}\right)$ in $T_{z} W^{\alpha} \times f_{t}^{a p p, *} T(z \times V)$, we denote by $\pi_{t}\left(u_{t 1}, u_{t 2} ; x\right)$ the parallel transport from $T_{f_{t}(x)}\left(z_{t} \times V\right)$ to $T_{f_{t}^{a p p}(x)}(z \times V)$ with respect to $\nabla$ along the path $\left(s u_{t 1}, e x p_{f_{t}^{a p p}(x)}^{*}\left(s u_{t 1}, s u_{t 2}\left(f_{t 1}^{a p p}(x)\right)\right)\right)_{1 \leq s \leq 1}$. Since $\nabla$ is $J$-compatible, we have

$$
J_{z} \circ \pi_{t}\left(u_{t 1}, u_{t 2} ; x\right)=\pi_{t}\left(u_{t 1}, u_{t 2} ; x\right) \circ J_{z_{t}} .
$$

Let $\Lambda^{0,1}\left(f_{t}^{a p p, *} T(z \times V)\right)$ be the vector bundle over $\Sigma_{t}$ of all anti- $\left(J_{z}, j_{t}\right)$-linear homomorphism from $T \Sigma_{t}$ to $f_{t}^{a p p, *} T(z \times V)$. Denote by $\Omega^{0,1}\left(f_{t}^{a p p, *} T(z \times V)\right)$ and $\Omega\left(f_{t}^{a p p, *} T(z \times V)\right)$ the space of sections of $\Lambda^{0,1}\left(f_{t}^{a p p *} T(z \times V)\right)$ and $f_{t}^{a p p, *} T(z \times V)$ respectively.

Define

$$
\begin{gathered}
\Phi_{t}: T_{z} W^{\alpha} \times \Omega\left(f_{t}^{a p p, *} T(z \times V)\right) \rightarrow \Omega^{0,1}\left(f_{t}^{a p p, *} T(z \times V)\right), \\
\left(u_{t 1}, u_{t 2}\right) \rightarrow \pi_{t}\left(u_{t 1}, u_{t 2} ; \cdot\right) \circ\left(d f_{t}+J_{z_{t}} \circ d f_{t} \circ j_{t}\right) .
\end{gathered}
$$

Because of (3.16), this map is well-defined. Let

$$
L_{t}^{v, a p p}(\sigma)=D \Phi_{t}(0) \sigma, \quad t \in \Delta .
$$

Lemma 3.3. For any $\sigma=\left(\sigma_{1}, \sigma_{2}\right) \in T_{z} W^{\alpha} \times \Omega\left(f_{t}^{a p p, *} T(z \times V)\right)$ and any $e \in T \Sigma_{t}$, we have

$$
\begin{aligned}
& L_{t}^{v, a p p}\left(\sigma_{1}, \sigma_{2}\right)(e)=\nabla_{e} \sigma_{2}+J_{z} \circ \nabla_{j_{t}} \sigma_{2}+\frac{1}{4} N_{j_{z}}\left(\partial_{J_{z}} f_{t}^{a p p}, \sigma_{2}\right) \\
& \quad+\left.\frac{d J_{z_{s}}}{d s}\right|_{s=0} \circ d f_{t}^{a p p} \circ j_{t} e+\Gamma\left(\sigma_{1}, v_{t}\right)
\end{aligned}
$$


where $z_{s}=s \sigma_{1}$ a path in $W^{\alpha}, \Gamma(\cdot, \cdot)$ is the Christoffel symbol of $\nabla$, and $v_{t}$ is defined in (3.13), $t \neq 0$. Here we identify $\Gamma\left(f_{t}^{a p p, *} T(z \times V)\right)$ with $\Gamma\left(f_{t}^{a p p}\left(\Sigma_{t}\right), T(z \times V)\right)$, so we may view $\nabla$ as a covariant derivative on $\Gamma\left(f_{t}^{a p p, *} T(z \times V)\right)$.

Proof. The computation, which is the same as we did in section 1.2 , is omitted.

Let $L_{t}^{v, a p p *}: \Omega^{0,1}\left(f_{t}^{a p p, *} T(z \times V)\right) \rightarrow T_{z} W^{\alpha} \times \Omega\left(f_{t}^{a p p, *} T(z \times V)\right)$ be the adjoint of $L_{t}^{v, a p p}$ with respect to the metric $d s_{0}^{2}, h_{E}$ and the metric $\mu_{c}$ on $\Sigma_{t}$. Let $\left(e, j_{t} e\right)$ be any local unitary basis of $T \Sigma_{t}$ with respect to $\mu_{c}, t \neq 0$.

Lemma 3.4. Assume that $\langle\cdot, \cdot\rangle$ is the induced metric on $f_{t}^{a p p, *} T(z \times V)$ by $h_{E}$. For any section $\xi \in \Omega^{0,1}\left(f_{t}^{a p p, *} T(z \times V)\right)$, we then have

$$
\begin{aligned}
L_{t}^{v, a p p *}(\xi)= & \left(2\left\langle\xi(e),(d J, \cdot) \circ d f_{t}^{a p p}\left(j_{t} e\right)+\Gamma\left(\cdot, v_{t}\right)\right\rangle^{*}\right. \\
& \left.-2 \nabla_{e} \xi(e)-2 \nabla_{j_{t}} \xi\left(j_{t} e\right)+\frac{1}{2}\left\langle\xi(e), N_{J_{z}}\left(\partial_{J_{z}} f_{t}^{a p p}, \cdot\right)(e)\right\rangle^{*}\right)
\end{aligned}
$$

Here $\left\langle\xi(e),(d J, \cdot) \circ d f_{t}^{a p p}\left(j_{t} e\right)+\Gamma\left(\cdot, v_{t}\right)\right\rangle^{*}$ is defined by the relation that its inner product with $\sigma_{1} \in T_{z} W^{\alpha}$ is $\int_{\Sigma_{t}}\left\langle\xi(e),\left.\frac{d J_{z_{s}}}{d s}\right|_{s=0} \circ d f_{t}^{a p p}\left(j_{t} e\right)+\right.$ $\left.\Gamma\left(\sigma_{1}, v_{t}\right)(e)\right\rangle d \mu_{c}$, and $\left\langle\xi(e), N_{J}\left(\partial_{J_{z}} f_{t}^{a p p}, \cdot\right)(e)\right\rangle^{*}$ is defined by the relation that its inner product with $\sigma_{2} \in f_{t}^{a p p, *} T(z \times V)$ is $\left\langle\xi(e), N_{J}\left(\partial_{J_{z}} f_{t}^{a p p}, \sigma_{2}\right)(e)\right\rangle$.

Proof. Let $e_{1}=e, e_{2}=j_{t} e$, then the dual basis $\left(e_{1}^{*}, e_{2}^{*}\right)$ satisfies $e_{2}^{*}=-j_{t} e_{1}^{*}$. Set

$$
\xi=\xi_{1} e_{1}^{*}+\xi_{2} e^{*}, \quad \xi_{i} \in f_{t}^{a p p, *} T(z \times V), \quad i=1,2 .
$$

Since $\xi$ is anti- $\left(J_{z}, j_{t}\right)$-linear, we have $\xi_{2}=-J_{z} \xi_{1}$. So for any anti- $\left(J_{z}, j_{t}\right)$ linear vector $X \in \Omega^{0,1}\left(f_{t}^{a p p, *} T(z \times V)\right)$, we have $\langle\xi, X\rangle=2\left\langle\xi_{1}, X\left(e_{1}\right)\right\rangle$. Using 
this, then

$$
\begin{aligned}
& \int_{\Sigma_{t}}\left\langle L_{t}^{v, a p p *} \xi, \sigma\right\rangle_{\mu_{c}, h_{E}} d \mu_{c} \\
& =\int_{\Sigma_{t}}\left\langle\xi, L_{t}^{v, a p p}\left(\sigma_{1}, \sigma_{2}\right)\right\rangle d \mu_{c} \\
& =2 \int_{\Sigma_{t}}\left\langle\xi_{1}, L_{t}^{v, a p p}\left(\sigma_{1}, \sigma_{2}\right)\left(e_{1}\right)\right\rangle d \mu_{c} \\
& =2 \int_{\Sigma_{t}}\left\langle\xi_{1}, \nabla_{e_{1}} \sigma_{2}+J_{z} \circ \nabla_{e_{2}} \sigma_{2}\right\rangle d \mu_{c}+\frac{1}{2} \int_{\Sigma_{t}}\left\langle\xi_{1}, N_{J_{z}}\left(\partial_{J_{z}} f_{t}^{a p p}, \sigma_{2}\right)\left(e_{1}\right)\right\rangle d \mu_{c} \\
& +2 \int_{\Sigma_{t}}\left\langle\xi 1,\left.\frac{\partial J_{z_{s}}}{d s}\right|_{s=0} \circ d f_{t}^{a p p}\left(e_{2}\right)+\Gamma\left(\sigma_{1}, v_{t}\right)\left(e_{1}\right)\right\rangle d \mu_{c} \\
& =-2 \int_{\Sigma_{t}}\left\langle\nabla_{e_{1}} \xi_{1}+\nabla_{e_{2}} \xi_{2}, \sigma_{2}\right\rangle d \mu_{c}+\frac{1}{2} \int_{\Sigma_{t}}\left\langle\xi_{1}, N_{J_{z}}\left(\partial_{J_{z}} f_{t}^{a p p}, \sigma_{2}\right)\left(e_{1}\right)\right\rangle d \mu_{c} \\
& \quad+2 \int_{\Sigma_{t}}\left\langle\xi_{1},\left.\frac{\partial J_{z_{s}}}{d s}\right|_{s=0} \circ d f_{t}^{a p p}\left(e_{2}\right)+\Gamma\left(\sigma_{1}, v_{t}\right)\left(e_{1}\right)\right\rangle d \mu_{c} .
\end{aligned}
$$

The lemma follows.

Lemma 3.5. Let $\xi \in \Omega^{0,1}\left(f_{t}^{a p p, *} T(z \times V)\right)$, and $r(\rho)$ be a positive function in $\rho$. For any $0<\epsilon<1$, there is a $C_{\epsilon}$ such that

$$
\begin{aligned}
& \int_{\Sigma_{t}} r(\rho)^{2}\left|L_{t}^{v, a p p *} \xi\right|_{\mu_{c}, h_{E}}^{2} d \mu_{c} \geq(2-\epsilon) \int_{\Sigma_{t}} r(\rho)^{2}|\nabla \xi|_{\mu_{c}, h_{E}}^{2} d \mu_{c} \\
& \quad-C_{\epsilon} \cdot \int_{\Sigma_{t}}\left(r(\rho)^{2}\left|d f_{t}^{a p p}\right|_{\mu_{c}, h_{E}}^{2} \cdot|\xi|_{\mu_{c}, h_{E}}^{2}+\left|r^{\prime}(\rho)\right|^{2} \cdot|\xi|_{\mu_{c}, h_{E}}^{2}\right) d \mu_{c}
\end{aligned}
$$

where $C_{\epsilon}$ depends only on the curvature tensor of $h_{E}, \mu_{c}$ and the fiberwise almost complex structure, $t \neq 0$.

Proof. Observe that

$$
\left\langle\xi(e),(d J, \cdot) \circ d f_{t}^{a p p}\left(j_{t} e\right)+\Gamma\left(\cdot, v_{t}\right)\right\rangle^{*}
$$

and

$$
\left\langle\xi(e), N_{J_{z}}\left(\partial_{J_{z}} f_{t}^{a p p}, \cdot\right)(e)\right\rangle^{*}
$$

are zero order operators of $\xi$. The computation, which is similar to the computation in the remark after Lemma 6.4 in [RT], is omitted. 
We will apply the implicit function theorem to construct the map $T_{t}$ in Theorem 3.1. First we need to establish the lower bound of the spectrum of $\square_{t}^{v, a p p}=L_{t}^{v, a p p} \circ L_{t}^{v, a p p *}$.

Lemma 3.6. Let $D$ be a disk in $\mathbb{C}$ and $\mu_{c}$ be the cylindrical metric on $D \backslash\{0\}$, i.e., $\mu_{c}=\frac{|d z|^{2}}{|z|^{2}}$. Suppose that $f_{D}: D \rightarrow z \times V$, where $z \in W^{\alpha}$, is a $J$-holomorphic map, $L_{D}^{v}$ is the linearization of Cauchy-Riemann equation at $f_{D}$ and $\xi$ is a $C^{2}$-smooth section of $\Omega^{0,1}\left(f_{D}^{*} T(z \times V)\right)$ over $D \backslash\{0\}$ satisfying $L_{D}^{v *} \xi=0$ on $D \backslash\{0\}$, where $L_{D}^{v *}$ is the adjoint of $L_{D}^{v}$ with respect to $\mu_{c}$. Furthermore

$$
\int_{D \backslash\{0\}}\left(|\nabla \xi|_{\mu_{c}, h_{E}}^{2}+|z|^{2} \cdot|\xi|_{\mu_{c}, h_{E}}^{2}\right) d \mu_{c}<\infty
$$

Then limit $\lim _{w \rightarrow 0} \xi(w)$ exists and is a vector in $T_{f_{D}(0)}(z \times V)$. Such a limit is called the residue of $\xi$ at $z=0$.

Proof. From Lemma 3.4, we know that $L_{D}^{v *} \xi=\left(L_{D 1}^{v *} \xi, L_{D 2}^{v *} \xi\right) \in T_{z} W^{\alpha} \times$ $\Omega^{0}\left(f_{D}^{*} T(z \times V)\right)$. Observe that $L_{D 2}^{v *}$ is exactly the operator $L_{0}^{*}$ in [RT]. Then the lemma follows from Lemma 6.5 in [RT].

Let $L_{0}^{v}$ and $L^{v}$ be the linearization of the Cauchy-Riemann equation at $f_{0}$ and $f$ respectively, and let $L_{0}^{v *}$ and $L^{v *}$ be the adjoints of $L_{0}^{v}$ and $L^{v}$ on $\Sigma_{0} \backslash \operatorname{Sing}\left(\Sigma_{0}\right)$. We denote by $\operatorname{Ker} L_{0}^{v *}$ the set of those sections $\xi$ of $\Omega^{0,1}\left(f_{0}^{*} T\left(z_{0} \times V\right)\right)$ over $\Sigma_{0} \backslash \operatorname{Sing}\left(\Sigma_{0}\right)$ satisfying $L_{0}^{v *} \xi=0$ outside $\operatorname{Sing}\left(\Sigma_{0}\right)$,

$$
\int_{\Sigma_{0}}|\nabla \xi|_{\mu_{c}, h_{E}}^{2}+\rho^{2} \cdot|\xi|_{\mu_{c}, h_{E}}^{2} d \mu_{c}<\infty
$$

and for every node $p$ in $\operatorname{Sing}\left(\Sigma_{0}\right)$

$$
\lim _{z \rightarrow 0, z \in U_{p 1}} \xi_{z}+\lim _{z \rightarrow 0, z \in U_{p 2}} \xi_{z}=0 .
$$

Here $U_{p}$ is any small neighborhood of $p$ with two irreducible components $U_{p 1}, U_{p 2}$. We define $\operatorname{Ker} L^{v *}$ similarly.

Proposition 3.1. For a generic $(J, \nu), \operatorname{KerL}_{0}^{v *}$ is trivial, and for any $f \in$ $\tilde{W}, k^{2} L^{v *}$ is trivial also.

Proof. Fact 1. Write $L_{0}^{v *}=\left(L_{01}^{v *}, L_{02}^{v *}\right)$, and $\operatorname{Ker} L_{0}^{v *} \subset \operatorname{Ker} L_{02}^{v *}$. According to Lemma 6.1 in [RT], $\operatorname{Ker} L_{02}^{v *}=0$, so $\operatorname{Ker} L_{0}^{v *}$ is trivial. 
Fact 2.Again $\Sigma_{0 i}$ are components of $\Sigma_{0}$. Let $\Sigma_{0 i}^{0}=\Sigma_{0 i} \backslash \operatorname{Sing}\left(\Sigma_{0}\right)$ and denote by $\left.L_{0}^{v *}\right|_{\Sigma_{0 i}^{0}}$ and $\left.L^{v *}\right|_{\Sigma_{0 i}^{0}}$ the restriction of $L_{0}^{v *}$ and $L^{v *}$ to $\Omega^{0,1}\left(\Sigma_{0 i}^{0}, f_{0}^{*} T\left(z_{0} \times\right.\right.$ $V)$ ) and $\Omega^{0,1}\left(\Sigma_{0 i}^{0}, f^{*} T(z \times V)\right)$ respectively. Since for elliptic operators, $\left.\operatorname{dim} \operatorname{Ker} L^{v *}\right|_{\Sigma_{0 i}^{0}}$ is upper semi-continuous function of $f,\left.\operatorname{dim} \operatorname{Ker} L^{v *}\right|_{\Sigma_{0 i}^{0}} \leq$ $\left.\operatorname{dim} \operatorname{Ker} L_{0}^{v *}\right|_{\Sigma_{0 i}^{0}} ^{0}$. Here we probably need to shrink $\tilde{W}$ a little bit.

Fact 3. Consider

$$
\begin{aligned}
& R_{0}: \prod_{i=1}^{m} \operatorname{Ker}\left(\left.L_{0}^{v *}\right|_{\Sigma_{0 i}^{0}}\right) \rightarrow \prod_{p \in \operatorname{Sing}\left(\Sigma_{0}\right)} T_{f_{0}(p)}\left(z_{0} \times V\right) \times T_{f_{0}(p)}\left(z_{0} \times V\right), \\
& R_{0}(\xi)=\prod_{p \in \operatorname{Sing}\left(\Sigma_{0}\right)}\left(\lim _{z \rightarrow p} \xi_{\Sigma_{0 i}^{0}}, \lim _{z \rightarrow p} \xi_{\Sigma_{\Sigma^{\prime}}^{0}}\right), \\
& \Delta_{0}=\prod_{p \in \operatorname{Sing}\left(\Sigma_{0}\right)}\left\{(u,-u) \mid u \in T_{f_{0}(p)}\left(z_{0} \times V\right)\right\}, \\
& R: \prod_{i=1}^{m} \operatorname{Ker}\left(\left.L^{v *}\right|_{\Sigma_{0 i}^{0}}\right) \rightarrow \prod_{p \in \operatorname{Sing}\left(\Sigma_{0}\right)} T_{f(p)}(z \times V) \times T_{f(p)}(z \times V), \\
& R(\xi)=\prod_{p \in \operatorname{Sing}\left(\Sigma_{0}\right)}\left(\lim _{z \rightarrow p} \xi_{\Sigma_{0 i}^{0}}, \lim m_{z \rightarrow p} \xi_{\Sigma_{0 i^{\prime}}^{0}}\right), \\
& \Delta=\prod_{p \in \operatorname{Sing}\left(\Sigma_{0}\right)}\left\{(u,-u) \mid u \in T_{f(p)}(z \times V)\right\} .
\end{aligned}
$$

On each component $\Sigma_{0 i}^{0}, f$ converges to $f_{0}$ in $C^{4}$-topology, and $\left.\operatorname{Ker} L^{v *}\right|_{\Sigma_{0 i}^{0}}$ converges to $\left.\operatorname{Ker} L_{0}^{v *}\right|_{\Sigma_{0 i}^{0}}$ in $C^{2}$-topology, i.e., $\operatorname{map} R$ is $C^{2}$-close to $R_{0}$. From the proof of Proposition 6.1 in [RT], map $R_{0}$ is transversal to $\Delta_{0}$ for generic $J, R$ map is transversal to $\Delta$. $\operatorname{Ker} L^{v *}=0$ follows from above three facts and a simple dimension counting.

Lemma 3.7. There is a constant $C>0$, independent of $t$, such that for $t$ sufficiently small, the first eigenvalue $\lambda_{1}\left(\square_{t}^{v, a p p}\right)$ of $\square_{t}^{v, a p p}$ is bounded from below by $\frac{C}{(\log |t|)^{2}}$.

Proof. Write $L_{t}^{v, a p p}=L_{t 1}^{v, a p p}+L_{t 2}^{v, a p p}$, where

$$
L_{t 1}^{v, a p p}: T_{z} W^{\alpha} \rightarrow \Omega^{0,1}\left(\Sigma_{t}, f_{t}^{a p p, *} T(z \times V)\right)
$$

and

$$
L_{t, a p p}^{v 2}: \Omega\left(\Sigma_{t}, f_{t}^{a p p, *} T(z \times V)\right) \rightarrow \Omega^{0,1}\left(\Sigma_{t}, f_{t}^{a p p, *} T(z \times V)\right) .
$$


Then $L_{t}^{v, a p p *}=\left(L_{t 1}^{v, a p p *}, L_{t 2}^{v, a p p *}\right)$, and $\square_{t}^{v, a p p}=L_{t 1}^{v, a p p} \circ L_{t 1}^{v, a p p *}+L_{t 2}^{v, a p p} \circ$ $L_{t 2}^{v, a p p *}$. Note that $L_{t 2}^{v, a p p} \circ L_{t 2}^{v, a p p *}$ is exactly the operator $L_{t} \circ L_{t}^{*}=\square_{t}$ in [RT]. Lemma 6.6 in [RT] then implies that the first eigenvalue $\lambda_{1}\left(L_{t} \circ L_{t}^{*}\right) \geq$ $\frac{C}{(\log |t|)^{2}}$. Note in the proof of Lemma $6.6[\mathrm{RT}]$, we need the second half of Proposition 3.1. Since operator $L_{t 1}^{v, a p p} \circ L_{t 1}^{v, a p p *}$ is non-negative, and the spectrum of $L_{t 2}^{v, a p p} \circ L_{t 2}^{v, a p p *}$ are all eigenvalues, $\lambda_{1}\left(\square_{t}^{v, a p p}\right) \geq \frac{C}{(l o g|t|)^{2}}$ follows from functional analysis.

Let $\Phi_{t}: T_{z} W^{\alpha} \times \Omega\left(f_{t}^{a p p, *} T(z \times V)\right) \rightarrow \Omega^{0,1}\left(f_{t}^{a p p, *} T(z \times V)\right)$ be the map defined in (3.17), define

$$
\Psi_{t}(\xi)=\Phi_{t} \circ L_{t}^{v, a p p *}: \Omega^{0,1}\left(f_{t}^{a p p, *} T(z \times V)\right) \rightarrow \Omega^{0,1}\left(f_{t}^{a p p, *} T(z \times V)\right) .
$$

To find a $J$-holomorphic $f_{t}$, it suffices to show that $\Psi_{t}(\xi)$ has a zero solution $\xi$.

Lemma 3.8. $\Psi_{t}(\xi)$ has the following expansion,

$$
\begin{aligned}
& \Psi_{t}(0)=v_{t} \\
& \Psi_{t}(\xi)=v_{t}+L_{t}^{v, a p p} \circ L_{t}^{v, a p p *} \xi+H_{t}(\xi)
\end{aligned}
$$

where $v_{t}$ is defined in (3.13). Furthermore, $H_{t}(\xi)$ satisfies

$$
\begin{aligned}
& \left\|H_{t}\left(\xi_{1}\right)-H_{t}\left(\xi_{2}\right)\right\|_{0, \frac{1}{2}} \leq C\left(\left\|\xi_{1}\right\|_{2, \frac{1}{2}}+\left\|\xi_{2}\right\|_{2, \frac{1}{2}}\right) \cdot\left\|\xi_{1}-\xi_{2}\right\|_{2, \frac{1}{2}} \\
& \left\|H_{t}(\xi)\right\|_{0, \frac{1}{2}} \leq C \cdot\|\xi\|_{1, \frac{1}{2}} \cdot\|\xi\|_{2, \frac{1}{2}}
\end{aligned}
$$

where we denote by $\|\cdot\|_{k, \frac{1}{2}}$ the $C^{k, \frac{1}{2}}$-Hölder norm defined by the metric $h_{\alpha}$ on $W^{\alpha} \times V$ and the metric $\mu_{c}$ on $\Sigma_{t}$.

Proof. Consider the expansion of $\Phi_{t}\left(\sigma_{1}, \sigma_{2}\right)$,

$$
\Phi_{t}\left(\sigma_{1}, \sigma_{2}\right)=v_{t}+L_{t 1}^{v, a p p} \sigma_{1}+L_{t 2}^{v, a p p} \sigma_{2}+G\left(\sigma_{1}, \sigma_{2}\right)
$$

where $G\left(\sigma_{1}, \sigma_{2}\right)$ is the nonlinear part. 


\section{Claim:}

$$
\begin{aligned}
& G\left(\sigma_{1}, \sigma_{2}\right)(e) \\
& =L_{1}\left(\sigma_{1}, \sigma_{2}\right) \circ \nabla_{e} \sigma_{2}+L_{2}\left(\sigma_{1}, \sigma_{2}\right) \circ \nabla_{j_{t} e} \sigma_{2} \\
& \quad+Q_{1}\left(\sigma_{1}, \sigma_{2}\right) \circ d f_{t}^{a p p}(e)+Q_{2}\left(\sigma_{1}, \sigma_{2}\right) \circ d f_{t}^{a p p}\left(j_{t} e\right), \\
& \left\|L_{i}\left(\sigma_{1}, \sigma_{2}\right)\right\|_{0, \frac{1}{2}} \leq C \cdot\left(\left\|\sigma_{1}\right\|_{0}+\left\|\sigma_{2}\right\|_{1, \frac{1}{2}}\right), \quad i=1,2, \\
& \left\|Q_{i}\left(\sigma_{1}, \sigma_{2}\right)\right\|_{0, \frac{1}{2}} \leq C \cdot\left(\left\|\sigma_{1}\right\|_{0}^{2}+\left\|\sigma_{2}\right\|_{1, \frac{1}{2}}^{2}\right), \quad i=1,2, \\
& \left\|G\left(\sigma_{1}, \sigma_{2}\right)-G\left(\sigma_{1}^{\prime}, \sigma_{2}^{\prime}\right)\right\|_{0, \frac{1}{2}} \\
& \leq C \cdot\left(\left\|\left(\sigma_{1}, \sigma_{2}\right)\right\|_{1, \frac{1}{2}}+\left\|\left(\sigma_{1}^{\prime}, \sigma_{2}^{\prime}\right)\right\|_{1, \frac{1}{2}}\right) \cdot\left\|\left(\sigma_{1}, \sigma_{2}\right)-\left(\sigma_{1}^{\prime}, \sigma_{2}^{\prime}\right)\right\|_{1, \frac{1}{2}}, \\
& \left\|G\left(\sigma_{1}, \sigma_{2}\right)\right\|_{0, \frac{1}{2}} \leq C \cdot\left\|\left(\sigma_{1}, \sigma_{2}\right)\right\|_{0, \frac{1}{2}} \cdot\left\|\left(\sigma_{1}, \sigma_{2}\right)\right\|_{1, \frac{1}{2}},
\end{aligned}
$$

where $e$ is a vector in $T \Sigma_{t}, L_{1}$ and $L_{2}$ are linear first order differential operator in $\left(\sigma_{1}, \sigma_{2}\right), Q_{1}\left(\sigma_{1}, \sigma_{2}\right)$ and $Q_{2}\left(\sigma_{1}, \sigma_{2}\right)$ are quadratic operators in $\left(\sigma_{1}, \sigma_{2}, \nabla \sigma_{2}\right)$.

To prove the claim, view $W^{\alpha} \times V$ as a symplectic manifold with standard symplectic form on $W^{\alpha} \subset \mathbb{R}^{\tilde{n}}$ (if $W^{\alpha}$ is odd dimension, we replace it by $\left.W^{\alpha} \times \mathbb{R}\right)$. Then $\Phi_{t}\left(\sigma_{1}, \sigma_{2}\right)$ is operator $\bar{\partial}$ at $f_{t}^{a p p}$ for symplectic manifold $W^{\alpha} \times V$. The claim follows from the calculation done by A. Floer [F1, section 3]. Note although the constant $C$ depends on $f_{t}^{a p p}$, since $\left\|f_{t}^{a p p}\right\|_{5, \frac{1}{2}}$ is bounded, we may assume constant $C$ does not depend on $f_{t}^{a p p}$ at all.

By choosing $\sigma_{1}=L_{t 1}^{v, a p p *} \xi, \sigma_{2}=L_{t 2}^{v, a p p *} \xi$ in the claim, note $\left\|\sigma_{1}\right\|_{0} \leq$ $C \cdot\|\xi\|_{1,0},\left\|\sigma_{2}\right\|_{1, \frac{1}{2}} \leq C \cdot\|\xi\|_{2, \frac{1}{2}}$, all the estimates of the lemma then follow from the claim.

Define

$$
\Gamma^{k, \frac{1}{2}}\left(\Lambda^{0,1} f_{t}^{a p p, *} T(z \times V)\right)=\left\{\xi \in \Omega^{0,1}\left(f_{t}^{a p p, *} T(z \times V)\right) \mid\|\xi\|_{k, \frac{1}{2}}<\infty\right\}
$$

Then

$$
\begin{aligned}
& \Psi_{t}: \Gamma^{2, \frac{1}{2}}\left(\Lambda^{0,1} f_{t}^{a p p, *} T(z \times V)\right) \rightarrow \Gamma^{0, \frac{1}{2}}\left(\Lambda^{0,1} f_{t}^{a p p, *} T(z \times V)\right), \\
& \square_{t}^{v, a p p}: \Gamma^{2, \frac{1}{2}}\left(\Lambda^{0,1} f_{t}^{a p p, *} T(z \times V)\right) \rightarrow \Gamma^{0, \frac{1}{2}}\left(\Lambda^{0,1} f_{t}^{a p p, *} T(z \times V)\right) .
\end{aligned}
$$

Lemma 3.9. Assume $\xi \in \Gamma^{2, \frac{1}{2}}\left(\Lambda^{0,1} f_{t}^{a p p, *} T(z \times V)\right), \zeta=\square_{t}^{v, a p p} \xi$ on $\Sigma_{t}$. Then

$$
\|\xi\|_{2, \frac{1}{2}} \leq C \cdot(-\log |t|)^{\frac{5}{2}}\|\zeta\|_{0, \frac{1}{2}}
$$


Proof. Note that

$$
\begin{aligned}
\operatorname{Vol}\left(\Sigma_{t}, \mu_{c}\right) & \leq C \cdot(-\log |t|) \\
\int_{\Sigma_{t}}|\xi|_{0}^{2} d \mu_{c} & \leq C \cdot\|\xi\|_{0}^{2} \cdot(-\log |t|) \\
\int_{\Sigma_{t}}\left|L_{t}^{v, a p p *} \xi\right|^{2} d \mu_{c} & =\int_{\Sigma_{t}}\left\langle\xi, \square_{t}^{v, a p p} \xi\right\rangle d \mu_{c} \quad=\int_{\Sigma_{t}}\langle\xi, \zeta\rangle d \mu_{c} \\
& \leq C \cdot\|\xi\|_{0}(-\log |t|)^{\frac{1}{2}} \cdot\left(\int_{\Sigma_{t}}|\zeta|_{0}^{2} d \mu_{c}\right)^{\frac{1}{2}}
\end{aligned}
$$

Lemma 3.7 implies that

$$
\int_{\Sigma_{t}}\left\langle\xi, \square_{t}^{v, a p p} \xi\right\rangle d \mu_{c} \geq \lambda_{1}\left(\square_{t}^{v, a p p}\right) \cdot \int_{\Sigma_{t}}|\xi|_{0}^{2} d \mu_{c}
$$

Then

$$
\int_{\Sigma_{t}}|\xi|_{0}^{2} d \mu_{c} \leq C \cdot\|\zeta\|_{0}^{2} \cdot(-\log |t|)^{5}
$$

Then (3.27) follows from above inequality and the standard elliptic estimates.

Proposition 3.2. There is a $t_{0}>0$ such that for any $0<|t|<\left|t_{0}\right|$, there is an unique $\xi \in \Gamma^{2, \frac{1}{2}}\left(\Lambda^{0,1} f_{t}^{a p p, *} T(z \times V)\right)$ satisfying $\|\xi\|_{2, \frac{1}{2}} \leq \sqrt{|t|}$ and $\Psi_{t}(\xi)=0$ on $\Sigma_{t}$, i.e., $f_{t}=\exp _{f_{t}^{a p p}}^{*}\left(L_{t}^{v, a p p *} \xi\right)$ is a J-holomorphic map from $\Sigma_{t}$ into $\tilde{z} \times V$ for some $\tilde{z} \in W^{\alpha}$. Denote this solution by $f_{t}^{\text {sol }}$ in the following.

Proof. Let $B \sqrt{|t|}(0)$ be the ball in $\Gamma^{2, \frac{1}{2}}\left(\Lambda^{0,1} f_{t}^{a p p, *} T(z \times V)\right)$ with radius $\sqrt{|t|}$ and the center at origin. Then solving equation $\Psi_{t}(\xi)=0$ for some $\xi \in$ $B_{\sqrt{|t|}}(0)$ is equivalent to solving equation

$$
\xi=\left(\square_{t}^{v, a p p}\right)^{-1}\left(-v_{t}+H_{t}(\xi)\right),
$$

where $\left(\square_{t}^{v, a p p}\right)^{-1}$ is the inverse of $\square_{t}^{v, a p p}$. Note that

$$
\begin{aligned}
\left(\square_{t}^{v, a p p}\right)^{-1}\left(-v_{t}+H_{t}(\cdot)\right): & \Gamma^{2, \frac{1}{2}}\left(\Lambda^{0,1} f_{t}^{a p p, *} T(z \times V)\right) \rightarrow \\
& \Gamma^{2, \frac{1}{2}}\left(\Lambda^{0,1} f_{t}^{a p p, *} T(z \times V)\right) .
\end{aligned}
$$



3.8 ,

First we show $\left(\square_{t}^{v, a p p}\right)^{-1}\left(-v_{t}+H_{t}(\cdot)\right): B_{\sqrt{|t|}}(0) \rightarrow B_{\sqrt{|t|}}(0)$. By lemma

$$
\begin{aligned}
& \left\|\left(\square_{t}^{v, a p p}\right)^{-1}\left(-v_{t}+H_{t}(\xi)\right)\right\|_{2, \frac{1}{2}} \\
& \leq C \cdot(-\log |t|)^{\frac{5}{2}} \cdot\left\|v_{t}\right\|_{0, \frac{1}{2}}+C \cdot(-\log |t|)^{\frac{5}{2}} \cdot\left\|H_{t}(\xi)\right\|_{0, \frac{1}{2}} \\
& \leq C \cdot(-\log |t|)^{\frac{5}{2}} \cdot|t|+C \cdot(-\log |t|)^{\frac{5}{2}} \cdot\|\xi\|_{1, \frac{1}{2}} \cdot\|\xi\|_{2, \frac{1}{2}} \\
& \leq C \cdot(-\log |t|)^{\frac{5}{2}} \cdot \sqrt{|t|} \cdot \sqrt{|t|} \leq \sqrt{|t|} .
\end{aligned}
$$

when $|t|$ is small enough.

Secondly we show $\left(\square_{t}^{v, a p p}\right)^{-1}\left(-v_{t}+H_{t}(\cdot)\right)$ is a contracting map.

$$
\begin{aligned}
& \left\|\left(\square_{t}^{v, a p p}\right)^{-1}\left(-v_{t}+H_{t}\left(\xi_{1}\right)\right)-\left(\square_{t}^{v, a p p}\right)^{-1}\left(-v_{t}+H_{t}\left(\xi_{2}\right)\right)\right\|_{2, \frac{1}{2}} \\
& \leq C \cdot(-\log |t|)^{\frac{5}{2}} \cdot\left\|H_{t}\left(\xi_{1}\right)-H_{t}\left(\xi_{2}\right)\right\|_{0, \frac{1}{2}} \\
& \leq C \cdot(-\log |t|)^{\frac{5}{2}} \cdot\left(\left\|\xi_{1}\right\|_{2, \frac{1}{2}}+\left\|\xi_{2}\right\|_{2, \frac{1}{2}}\right) \cdot\left\|\xi_{1}-\xi_{2}\right\|_{2, \frac{1}{2}} \\
& \leq C \cdot(-\log |t|)^{\frac{5}{2}} \cdot \sqrt{|t|} \cdot\left\|\xi_{1}-\xi_{2}\right\|_{2, \frac{1}{2}} \cdot
\end{aligned}
$$

When $|t|$ is small enough, it is a contraction map. So by the Banach contracting mapping theorem, $\left(\square_{t}^{v, a p p}\right)^{-1}\left(-v_{t}+H_{t}(\cdot)\right)$ has an unique fixed point. The proposition is proved.

Assume that $f_{0} \in \mathcal{M}_{\left(A_{1}, \cdots, A_{m}\right)}^{v}\left(\Sigma_{0}, \mathcal{X}_{W^{\alpha}}, J, 0\right)$. By the transversality result in section 1.2 , the tangent space of $\mathcal{M}_{\left(A_{1}, \cdots, A_{m}\right)}^{v}\left(\Sigma_{0}, \mathcal{X}_{W^{\alpha}}, J, 0\right)$ at $f_{0}$ is naturally identified with the kernel $\operatorname{Ker} L_{0}^{v}$, where $L_{0}^{v}$ is the linearization of the Cauchy-Riemann equation at $f_{0}$, i.e., a tangent vector at $f_{0}$ is a continuous section $u=\left(u_{1}, u_{2}\right) \in T_{z_{0}} W^{\alpha} \times \Omega\left(\Sigma_{0}, f_{0}^{*} T\left(z_{0} \times V\right)\right.$ satisfying $L_{0}^{v} u=0$ on $\Sigma_{0} \backslash \operatorname{Sing}\left(\Sigma_{0}\right)$. This implies that there is a local diffeomorphism from a neighborhood of 0 in $\operatorname{Ker} L_{0}^{v}$ into $\mathcal{M}_{\left(A_{1}, \cdots, A_{m}\right)}^{v}\left(\Sigma_{0}, \mathcal{X}_{W^{\alpha}}, J, 0\right)$. We assume that $\tilde{W}$ is contained in the image of such a neighborhood, we may assume that for any $f_{a}$ and $f_{b}$ in $\tilde{W}$,

$$
\left\|f_{a}-f_{b}\right\|_{C^{4}\left(\Sigma_{0}\right)} \leq C \cdot\left\|f_{a}-f_{b}\right\|_{C^{0}\left(\Sigma_{0}\right)} .
$$

Given any $f$ in $\tilde{W}$, there is a unique section $u_{f t}^{a p p}=\left(u_{f t 1}^{a p p}, u_{f t 2}^{a p p}\right)$ in $T_{z_{0}} W^{\alpha} \times \Omega\left(\Sigma_{0}, f_{0 t}^{a p p, *} T\left(z_{0} \times V\right)\right)$ such that

$$
f_{t}^{a p p}(x)=\exp _{f_{0 t}^{a p p}(x)}^{*}\left(u_{f t}^{a p p}\left(f_{0 t}^{a p p}(x)\right)\right), \quad x \in \Sigma_{t},
$$


where $f_{0 t}^{a p p}(x)$ is the approximate solution constructed from $f_{0}$. A straightforward computation shows

$$
\left|\nabla u_{f t 2}^{a p p}\right|_{\mu_{c}, h_{E}}(x) \leq C \cdot \rho(x),
$$

and for any $f_{a}, f_{b}$ in $\tilde{W}$

$$
\left\|u_{f_{a} t}^{a p p}-u_{f_{b} t}^{a p p}\right\|_{C^{4}\left(\Sigma_{t}\right)} \leq C \cdot\left\|f_{a}-f_{b}\right\|_{C^{0}\left(\Sigma_{0}\right)}
$$

We define $T_{t}: \tilde{W} \rightarrow \mathcal{M}_{A}^{v}\left(\Sigma_{t}, \mathcal{X}_{W^{\alpha}}, J, 0\right)$ by assigning $f_{t}^{s o l}$ in Proposition 3.2 to each $f$ in $\tilde{W}$. Clearly, $T_{t}(f)$ converges to $f$ as $t$ goes to zero. It is easy to see that $T_{t}$ is smooth. We want to examine the invertibility of the differential of $T_{t}$ at any point $f$ in $\tilde{W}$. For simplicity, we do it at $f_{0}$.

Lemma 3.10. Let $\pi_{t}\left(u_{f t}^{a p p}, x\right)$ be the parallel transport along the path $\left\{\exp _{f_{0 t}^{a p p}(x)}^{*}\left(s u_{f t}^{a p p}\left(f_{0 t}^{a p p}(x)\right)\right)\right\}_{0 \leq s \leq 1}$. Then there is a uniform constant $C>0$ such that

$$
\left\|v_{0 t}-\pi_{t}\left(u_{f t}^{a p p}, x\right) v_{t}\right\|_{C^{0}} \leq C \cdot \sqrt{|t|} \cdot\left\|f-f_{0}\right\|_{C^{0}\left(\Sigma_{0}\right)},
$$

where $v_{0 t}=d f_{0 t}^{a p p}+J_{z_{0}} \circ d f_{0 t}^{a p p} \circ j_{t}$.

Proof. Choose a diffeomorphism $\phi$ from a neighborhood of $f_{0 t}^{a p p}\left(\Sigma_{t}\right)$ onto a neighborhood of $f_{t}^{a p p}\left(\Sigma_{t}\right)$ satisfying (1) $\|\phi-i d\|_{C^{4}} \leq\left\|f_{0}-f\right\|_{C^{5}}$; (2) for each node $p$ in $\operatorname{Sing}\left(\Sigma_{0}\right)$, let $U_{p}, z_{p 1}$ and $z_{p 2}$ be as in the begining of this subsection, then

$$
f_{0 t}^{a p p}\left(z_{p 1}, z_{p 2}\right)=\phi^{-1} \circ f_{t}^{a p p}\left(z_{p 1}, z_{p 2}\right)=\left(z_{0}, z_{p 1}, z_{p 2}, 0, \cdots, 0\right) \quad \in z_{0} \times V,
$$

for all $\left(z_{p 1}, z_{p 2}\right)$ in $U_{p}$.

When $x \in \Sigma_{t} \backslash\left\{\cup_{p \in \operatorname{Sing} \Sigma_{0}} U_{p}\right\}$, then by the definition of $f_{0 t}^{a p p}$ and $f_{t}^{a p p}$, we have

$$
f_{0 t}^{a p p}(x)=f_{0} \circ \phi_{t}^{-1}(x), \quad f_{t}^{a p p}(x)=f \circ \phi_{t}^{-1}(x),
$$

where $\phi_{t}^{-1}$ is the diffemorphism used in the construction of approximated solution. Since both $f$ and $f_{0}$ are $J$-holomorphic, we have

$$
\begin{aligned}
& v_{0 t}(x)=d f_{0 t}^{a p p}+J \circ d f_{0 t}^{a p p} \circ j_{t}=J_{z_{0}} \circ d f_{0} \circ\left(\phi_{t *}^{-1} \circ j_{t}-j_{0} \circ \phi_{t *}^{-1}\right), \\
& v_{t}(x)=J_{z} \circ d f \circ\left(\phi_{t *}^{-1} \circ j_{t}-j_{0} \circ \phi_{t *}^{-1}\right) .
\end{aligned}
$$


It follows that

$$
\begin{aligned}
& \left|v_{0 t}(x)-\phi_{*}^{-1} v_{t}(x)\right| \\
& =\left|\left(J_{z_{0}} \circ d f_{0}-\phi_{*}^{-1} \circ J_{z} \circ d f\right) \cdot\left(\phi_{t^{*}}^{-1} \circ j_{t}-j_{0} \circ \phi_{t *}^{-1}\right)\right|(x) \\
& \leq C \cdot\left\|f-f_{0}\right\|_{C^{1}} \cdot|t| .
\end{aligned}
$$

When $x$ is in $U_{p}$ where $p$ is a double point of $\Sigma_{0}$. Then $\phi^{-1} \circ f_{t}^{a p p}\left(z_{p 1}, z_{p 2}\right)=$ $f_{0 t}^{a p p}\left(z_{p 1}, z_{p 2}\right)=\left(z_{0}, z_{p 1}, z_{p 2}, 0, \cdots, 0\right)$ implies that

$$
\phi_{*}^{-1} v_{t}(x)=\left(\phi_{*}^{-1} \circ J_{z} \circ \phi_{*}-J_{0}\right) \circ d \phi^{-1} \circ f_{t}^{a p p} \circ j_{t},
$$

where $J_{0}$ is the standard complex structure on $\mathbb{C}^{n}$.

Since $\phi^{-1} \circ f$ is $\phi_{*}^{-1} \circ J \circ \phi_{*}$-holomorphic, we have

$$
\left.\phi_{*}^{-1} \circ J_{z} \circ \phi_{*}\right|_{f\left(z_{p 1}, 0\right)}=J_{0}=\left.\phi_{*}^{-1} \circ J_{z} \circ \phi_{*}\right|_{f\left(0, z_{p 2}\right)} .
$$

In $\mathbb{C}^{n}$, we view $\left.\phi_{*}^{-1} \circ J_{z} \circ \phi_{*}\right|_{f\left(z_{p 1}, 0\right)}$ as an almost complex structure at $f\left(z_{p 1}, z_{p 2}\right)$. We may assume that $\left|z_{p 1}\right| \geq\left|z_{p 2}\right|$ at $x$. It follows that

$$
\phi_{*}^{-1} v_{t}(x)=\left(\left.\phi_{*}^{-1} \circ J_{z} \circ \phi_{*}\right|_{f\left(z_{p 1}, z_{p 2}\right)}-\left.\phi_{*}^{-1} \circ J_{z} \circ \phi_{*}\right|_{f\left(z_{p 1}, 0\right)}\right) \circ d f_{0 t}^{a p p} \circ j_{t} .
$$

Similarly we also have

$$
v_{0 t}(x)=\left(\left.J_{z_{0}}\right|_{f_{0}\left(z_{p 1}, z_{p 2}\right)}-\left.J_{z_{0}}\right|_{f_{0}\left(z_{p 1}, 0\right)}\right) \circ d f_{0 t}^{a p p} \circ j_{t} .
$$

By the mean value theorem we deduce on $U_{p}$

$$
\begin{aligned}
& \left|v_{0 t}(x)-\phi_{*}^{-1} v_{t}(x)\right|_{\mu_{c}} \\
& =\sup _{0 \leq \epsilon \leq 1}\left|\nabla\left(J-\phi_{*}^{-1} \circ J \circ \phi_{*}\right)\right|_{f\left(z_{p 1}, z_{p 2}\right)} \cdot\left|z_{p 1}\right| \cdot\left|d f_{0 t}^{a p p}\right|(x) \\
& \leq C \cdot\left\|f-f_{0}\right\|_{c^{0}\left(\Sigma_{0}\right)} \cdot \sqrt{t} .
\end{aligned}
$$

It follows from the definition of approximate solution that

$$
\left|v_{0 t}(x)-v_{t}(x)\right| \leq C \cdot\left\|f-f_{0}\right\|_{C^{0}\left(\Sigma_{0}\right)} \cdot \sqrt{|t|} .
$$

The lemma follows from combining this inequality, $\left|v_{t}\right|_{C^{0}} \leq C \cdot|t|$, and $\left\|\pi_{t}^{-1}\left(u_{f t}^{a p p}, \cdot\right) \circ \phi_{*}^{-1}-i d\right\|_{C^{2}} \leq C \cdot\left\|f-f_{0}\right\|_{C^{0}}$.

Let $\xi_{1}, \xi_{2}$ be the sections in Proposition 3.2, such that $T_{t}\left(f_{0}\right)=$ $\exp _{f_{0 t}^{a p p}}^{*}\left(L_{0 t}^{v, a p p *} \xi_{1}\right)$, and $T_{t}(f)=\exp _{f_{t}^{a p p}}^{*}\left(L_{t}^{v, a p p *} \xi_{2}\right)$. Using the fact that 
$T_{t}\left(f_{0}\right), T_{t}(f)$ are $J$-holomorphic, we have

$$
\begin{aligned}
& 0=v_{0 t}+L_{0 t}^{v, a p p} \circ L_{0 t}^{v, a p p *}\left(\xi_{1}\right)+H_{0 t}\left(\xi_{1}\right), \\
& 0=\pi_{t}\left(u_{f t}^{a p p}, \cdot\right)\left(v_{t}+L_{t}^{v, a p p} \circ L_{t}^{v, a p p *}\left(\xi_{2}\right)+H_{0 t}\left(\xi_{2}\right)\right) \\
& \left\|\xi_{1}\right\|_{1, \frac{1}{2}} \leq \sqrt{|t|},\left\|\xi_{2}\right\|_{1, \frac{1}{2}} \leq \sqrt{|t|} .
\end{aligned}
$$

Note that

$$
\begin{aligned}
& \left\|\left(\pi_{t}\left(u_{f t}^{a p p}, \cdot\right) \circ L_{t}^{v, a p p} \circ L_{t}^{v, a p p *}-L_{0 t}^{v, a p p} \circ L_{0 t}^{v, a p p *} \circ \pi_{t}\left(u_{f t}^{a p p}, \cdot\right)\right) \xi_{2}\right\|_{0, \frac{1}{2}} \\
& \quad \leq C \cdot \sqrt{|t|} \cdot\left\|f-f_{0}\right\|_{C^{0}},
\end{aligned}
$$

$$
\left\|\pi_{t}\left(u_{f t}^{a p p}, \cdot\right) H_{t}\left(\xi_{2}\right)-H_{0 t}\left(\pi_{t}\left(u_{f t}^{a p p}, \cdot\right) \xi_{2}\right)\right\|_{0, \frac{1}{2}} \leq C \cdot \sqrt{|t|} \cdot\left\|f-f_{0}\right\|_{C^{0}} .
$$

Substracting (3.30) from (3.31), we obtain

$$
\begin{aligned}
& \pi_{t}\left(u_{f t}^{a p p}, \cdot\right) v_{t}-v_{0 t} \\
& =\square_{t}^{v}\left(\xi_{1}-\pi_{t}\left(u_{f t}^{a p p}, \cdot\right) \xi_{2}\right)+H_{0 t}\left(\xi_{1}\right)-H_{0 t}\left(\pi_{t}\left(u_{f t}^{a p p}, \cdot\right) \xi_{2}\right)+B
\end{aligned}
$$

(3.32) and (3.33) imply

$$
\|B\|_{0, \frac{1}{2}} \leq C \cdot \sqrt{|t|} \cdot\left\|f-f_{0}\right\|_{C^{0}}
$$

Applying Lemma 3.9 to (3.34), and using Lemma 3.10 and (3.35), we get

$$
\left\|\xi_{1}-\pi_{t}\left(u_{f t}^{a p p},\right) \xi_{2}\right\|_{2, \frac{1}{2}} \leq C \cdot \sqrt{|t|} \cdot(-\log |t|)^{\frac{5}{2}} \cdot\left\|f-f_{0}\right\|_{C^{0}} .
$$

Therefore the map $T_{t}$ is injective near $f_{0}$. Moreover, if $D_{f_{0}} T_{t}$ denotes the derivative of $T_{t}$ at $f_{0}$, then for any $u$ in $\operatorname{Ker} L_{0}^{v}$, we have

$$
\left(1-C \cdot|t|^{\frac{1}{4}}\right)\|u\|_{0} \leq\left\|D_{f_{0}} T_{t}(u)\right\|_{0} \leq\left(1+C \cdot|t|^{\frac{1}{4}}\right)\|u\|_{0} .
$$

Denote by $L_{0 t}^{v, s o l}$ the linearization of the Cauchy-Riemann equation at $f_{0 t}^{s o l}$. 
Lemma 3.11. Let $K e r L_{0 t}^{v, s o l}$ be the set of all solutions for $L_{0 t}^{v, s o l} u=0$. Then $K e r L_{0 t}^{v \text {,sol }}$ converges uniformly to KerL $L_{0}^{v}$ as $t$ goes to zero. In particular, the dimension of $K e r L_{0 t}^{v, s o l}$ stays as a constant for $t$ small enough.

Proof. The proof of the first part is similar to the proof of Lemma 6.9 in [RT]. Note that $\operatorname{Ker} L_{0 t}^{v, s o l} \subset T_{\tilde{z}} W^{\alpha} \times \Omega\left(f_{0 t}^{s o l *} T(\tilde{z} \times V)\right)$, one can show that $\operatorname{dim} \operatorname{Ker} L_{0 t}^{v, s o l}$ is a upper semi-continuous at $t=0$ as in Proposition 3.1. Then (3.36) implies it is actually continuous.

Corollary 3.1. The derivative $D_{f_{0}} T_{t}$ is an isomorphism between $\operatorname{Ker} L_{0}^{v}$ and $\mathrm{KerL}_{0 t}^{v \text {,sol }}$ satisfying (3.36).

Remark 3.1. Note that in non-fiberwise case $T_{t}$ is an orientation preserving map, and the $T_{\tilde{z}} W^{\alpha}$ direction in $\operatorname{Ker} L_{0 t}^{v \text {,sol }}$ has fixed orientation. So in fiberwise case $T_{t}$ is again an orientation preserving map.

It remains to show part (2) in the Theorem 3.1. Let $f^{\prime}$ be given as in Theorem 3.1. For any $f$ in $\tilde{W}$, then there is a unique vector field $u_{f_{t}^{\text {sol }}}=$ $\left(u_{f_{t}^{s o l} 1}, u_{f_{t}^{s o l} 2}\right)$ such that $f^{\prime}(x)=\exp _{f_{t}^{s o l}(x)}^{*}\left(u_{f_{t}^{s o l}}\left(f_{t}^{s o l}(x)\right)\right)$ and $\left\|u_{f_{t}^{s o l}}\right\|_{0} \leq \epsilon^{\prime}$, where $\epsilon^{\prime}$ is small and depends only on $\tilde{W}$ and $\epsilon$ in Theorem 3.1. We want to show that $f^{\prime}$ coincides with one of $f_{t}^{\text {sol }}$.

Lemma 3.12. Let $\rho$ be the function in Lemma 3.5, $F$ be either $f_{t}^{\text {sol }}$ or $f^{\prime}$. Then there is a uniform constant $\lambda<1$ such that

$$
\int_{-\frac{1}{2} \cdot \log |t| \geq-\log \rho \geq R}|d F|_{\mu_{c}, h_{E}}^{2} d \mu_{c} \leq 4 \lambda^{R} .
$$

Consequently, for some uniform $\beta_{0}>0$,

$$
|d F|_{\mu_{c}, h_{E}}^{2}(x) \leq C \cdot \rho(x)^{8 \beta_{0}}
$$

Proof. This is exactly Lemma 6.10 in [RT].

Lemma 3.13. If $\epsilon,|t|$ are sufficiently small, there is a $f$ in $\tilde{W}$ such that

$$
\left\|u_{f}\right\|_{1,0} \leq C \cdot|t|^{\beta_{0}}
$$

where $\beta_{0}$ is given in (3.38). 
Proof. By cutting $\Sigma_{t}$ along the loops in $\left\{x \in \Sigma_{t} \mid \rho(x)=\sqrt{2|t|}\right\}$ and gluing disks to the boundary components of the resulting surface, we obtain $m$ surfaces $\Sigma_{i t}, i=1, \cdots, m$. Let $\tilde{\Sigma}_{t}$ be the disjoint union of those surfaces. We can naturally embed $\Sigma_{t} \backslash\left\{x \in \Sigma_{t} \mid \rho(x)=\sqrt{2|t|}\right\}$ into $\tilde{\Sigma}_{t}$ as a submanifold. Then we can extend the conformal structure $j_{t}$ on $\Sigma_{t}$ to be a natural conformal structure $\tilde{j}_{t}$ on $\tilde{\Sigma}_{t}$.

It follows from Lemma 3.12 that when $2 \cdot \sqrt{|t|} \leq \rho(x) \leq 10 \cdot \sqrt{|t|}$,

$$
\left|d f^{\prime}\right|_{\mu_{c}, h_{E}}(x) \leq C \cdot|t|^{2 \beta_{0}} .
$$

Therefore we can extend $f^{\prime}$ to be a map $\tilde{f}$ from $\tilde{\Sigma}_{t}$ into $z \times V$ satisfying

$$
\|\tilde{v}\|_{0, \frac{1}{2}} \leq C \cdot|t|^{2 \beta_{0}}
$$

where $\tilde{v}=d \tilde{f}+J_{z} \circ d \tilde{f} \circ \tilde{j}_{t}$. We denote by $\tilde{L}^{v}$ the linearization of CauchyRiemann equation at $\tilde{f}$, by $\tilde{L}^{v *}$ its adjoint. Then by the same arguement as in the proof of Lemma 3.7, one can show that the first eigenvalue of $\tilde{L}^{v} \circ \tilde{L}^{v *}$ is no less than $C \cdot(-\log |t|)^{-2}$, where $C$ is independent of $f^{\prime}$ and $t$. By applying the implicit function theorem in a similar manner to the proof of Proposition 3.2, we can find $\xi \in T_{z} W^{\alpha} \times \Omega^{0,1}\left(\tilde{f}^{*} T(z \times V)\right)$ such that $\tilde{f}^{s o l}=\exp _{\tilde{f}}^{*}\left(\tilde{L}^{v *} \xi\right)$ is $J$-holomorphic. Moreover, if $|t|$ is sufficiently small, we have

$$
\|\xi\|_{2, \frac{1}{2}} \leq C \cdot|t|^{\frac{3}{2} \beta_{0}}
$$

So the distance between $\operatorname{Im}\left(f^{\prime}\right)$ and $\operatorname{Im}\left(\tilde{f}^{s o l}\right)$ is less than $C \cdot|t|^{\frac{3}{2} \beta_{0}}$. The map $\tilde{f}^{s o l}$ may not be in $\mathcal{M}_{\left(A_{1}, \cdots, A_{m}\right)}^{v}\left(\Sigma_{0}, \mathcal{X}_{Z^{\alpha}}, J, \nu_{0}\right)$. However, using (3.40) and the fact that $\mathcal{M}_{\left(A_{1}, \cdots, A_{m}\right)}^{v}\left(\Sigma_{0}, \mathcal{X}_{Z^{\alpha}}, J, \nu_{0}\right)$ is smooth at $f_{0}$, one can show that $\tilde{f}^{\text {sol }}$ lies in a $C \cdot|t|^{\beta_{0}}$ neighborhood of some $f_{t}$ in $\tilde{W}$, as long as both $|t|$ and $\epsilon$ are sufficiently small. The lemma is proved.

Without loss of generality, we assume that $f=f_{0}$ in Lemma 3.13. Let $\xi$ be the unique solution of $L_{0 t}^{v, \text { sol }} \circ L_{0 t}^{v, s o l *} \xi=L_{0 t}^{v, s o l} u_{f_{t}^{s o l}}$. Multiplying this equation by $\xi$ and integrating by parts, we obtain

$$
\int_{\Sigma_{t}}\left|L_{0 t}^{v, s o l *} \xi\right|^{2} d \mu_{c} \leq \sqrt{\int_{\Sigma_{t}}\left|L_{0 t}^{v, s o l} u_{f_{t}^{s o l}}\right|^{2} d \mu_{c} \cdot \int_{\Sigma_{t}}|\xi|^{2} d \mu_{c} .}
$$

By the same argument as in the proof of Lemma 3.9, one can show

$$
\int_{\Sigma_{t}}|\xi|^{2} d \mu_{c} \leq C \cdot(-\log |t|)^{\frac{5}{2}} \cdot \int_{\Sigma_{t}}\left|L_{0 t}^{v, s o l *} \xi\right|^{2} d \mu_{c}
$$


Combine with (3.39) and (3.41), we get for $t$ sufficiently small,

$$
\int_{\Sigma_{t}}|\xi|^{2} d \mu_{c} \leq C \cdot|t|^{\frac{\beta_{0}}{2}}
$$

Since $L_{0 t}^{v, \text { sol }} \circ L_{0 t}^{v, \text { sol } *} \xi=L_{0 t}^{v, \text { sol }} u_{f_{t}^{s o l}}=O\left(\left\|u_{f_{t}^{s o l}}\right\|_{1,0}\right)$, by the standard elliptic estimate we have

$$
\|\xi\|_{1, \frac{1}{2}} \leq C \cdot|t|^{\frac{\beta_{0}}{2}}
$$

Consequently

$$
\left\|u_{f_{t}^{s o l}}-L_{0 t}^{v, s o l *} \xi\right\|_{1, \frac{1}{2}} \leq C \cdot|t|^{\frac{\beta_{0}}{2}} .
$$

Now we want to find a new $f_{1}$ in $\tilde{W}$ which is very close to $f=f_{0}$, such that $u_{f_{1 t}^{s o l}}=L_{1 t}^{v, s o l *} \xi_{1}$ for some $\xi_{1} \in \Omega^{0,1}\left(\Sigma_{t}, f_{1 t}^{s o l}(W \times V)\right)$, where $L_{1 t}^{v, s o l}$ is linearization of $\bar{\partial}$ operator at $f_{1 t}^{s o l}$. Using equation $f^{\prime}=\exp _{f_{1 t}^{\text {sol }}}^{*}\left(u_{f_{1 t}^{\text {sol }}}\right)=$ $\exp _{f_{t}^{s o l}}^{*}\left(u_{f_{t}^{\text {sol }}}\right)$, we can define a map $S_{t}$ from a neighborhood of $f_{0 t}^{\text {sol }}$ into $\operatorname{Ker} L_{0 t}^{\text {sol }}$ at $f_{0 t}^{s o l}$,

$$
S_{t}\left(f_{1}\right)=\pi\left(f_{0}, f_{1}\right)\left(u_{f_{1 t}^{s o l}}-L_{1 t}^{v, \text { sol* }} \xi_{1}\right)
$$

where $L_{1 t}^{v, s o l} u_{f_{1}^{s o l}}=L_{1 t}^{v, s o l} \circ L_{1 t}^{v, \text { sol* }} \xi_{1}, \pi\left(f_{0}, f_{1}\right)$ denotes the isomorphism from $\operatorname{Ker} L_{1 t}^{v, s o l}$ at $f_{1 t}^{s o l}$ to $\operatorname{Ker} L_{0 t}^{\text {sol }}$ at $f_{0 t}$ which depends smoothly on $f_{1}$. Clearly $S_{t}$ is a smooth map. By the same argument as in the proof of (3.36), one can show

$$
\left(1-C \cdot|t|^{\frac{\beta_{0}}{4}}\right)\|u\|_{0} \leq\left\|D_{f} S_{t}(u)\right\|_{0} \leq\left(1+C \cdot|t|^{\frac{\beta_{0}}{4}}\right)\|u\|_{0}
$$

Then by the implicit function theorem, there is a $f_{1}$ such that $S_{t}\left(f_{1}\right)=0$, and $\left\|u_{f_{1 t}^{s o l}}\right\|_{1, \frac{1}{2}} \leq C \cdot|t|^{\frac{\beta_{0}}{8}}$. For simlicity, we may assume that $f_{1}$ coincides with $f_{0}$, then $u_{f_{0 t}^{s o l}}=L_{0 t}^{v, s o l *} \xi$. Since both $f^{\prime}$ and $f_{0}$ are $J$-holomorphic, it follows from Lemma 3.8 that

$$
\square_{0 t}^{v} \xi=-H_{t}(\xi)
$$

Multiplying this equation by $\xi$ and integrating by parts, one can deduce

$$
\int_{\Sigma_{t}}\left|L_{0 t}^{v, \text { sol* }} \xi\right|^{2} d \mu_{c} \leq C \cdot\left\|L_{0 t}^{v, \text { sol* }} \xi\right\|_{1} \cdot \int_{\Sigma_{t}}|\xi|^{2} d \mu_{c}
$$

However $\left\|L_{0 t}^{v, s o l *} \xi\right\|_{1} \leq C \cdot|t|^{\frac{\beta_{0}}{8}}$, so for $t$ small enough, (3.45) is impossible unless $u_{f_{t}^{s o l}}=L_{t}^{v, \text { sol } *} \xi=0$, i.e., $f^{\prime}=f_{0 t}$. Theorem is proved. 
Remark 3.2. Essentially Theorem 3.1 is a corollary of corresponding gluing theorem in non-fiberwise case. This can be seen as follows: view $W^{\alpha} \times V$ as a symplectic manifold, now fiberwise moduli space $\mathcal{M}_{\left(A_{1}, \cdots, A_{m}\right)}^{v}\left(\Sigma_{0}, \mathcal{X}_{W^{\alpha}}, J, 0\right)$ and $\mathcal{M}_{A}^{v}\left(\Sigma_{t}, \mathcal{X}_{W^{\alpha}}, J, 0\right)$ is a subspace of $\mathcal{M}_{\left(A_{1}, \cdots, A_{m}\right)}\left(\Sigma_{0}, W^{\alpha} \times V, J, 0\right)$ and $\mathcal{M}_{A}\left(\Sigma_{t}, W^{\alpha} \times V, J, 0\right)$. The key difference is that in the non-fiberwise case, the deformation space of $J$-holomorphic map $f$ is parametrized by subspace of $\Omega\left(f^{*} T\left(W^{\alpha} \times V\right)\right)$ whose $T W^{\alpha}$ component may not be a constant. Fortunately, by modifying exponential map and applying the requirement that $T W^{\alpha}$-component is a constant to non-fiberwise deformation theory, each deformed map is a fiberwise $J$ holomorphic map, which gives exactly fiberwise gluing theorem.

\subsection{Composition Law of Fiberwise Gromov-Witten Mixed Invariants.}

Let $\left(L_{i}, U_{i}\right)_{i=1}^{k}$ and $\left(M_{j}, W_{j}\right)_{j=1}^{l}$ be pseudo-manifolds in $\mathcal{X}_{Z}$ satisfying the general position condition. Assume

$$
\begin{aligned}
& \sum_{i=1}^{k}\left(2 n+\operatorname{dim} Z-\operatorname{dim} U_{i}\right)+\sum_{j=1}^{l}\left(2 n+\operatorname{dim} Z-\operatorname{dim} W_{j}-2\right) \\
& =2 C_{1}(V) \cdot A+2 n(1-g)+\operatorname{dim} Z,
\end{aligned}
$$

where $g$ is the genus of a smooth Riemann surface $\Sigma_{t}$.

Adopting the notations at the beginning of section 3.1, define the evaluation map for $t \neq 0$

$$
\begin{aligned}
& e_{t}: \mathcal{M}_{A}^{v}\left(\Sigma_{t}, \mathcal{X}_{Z}, J, \nu_{t}\right) \times\left(\Sigma_{t}\right)^{l} \rightarrow \mathcal{X}_{Z}^{k+l} \\
& e_{t}\left(f ; y_{1}, \cdots, y_{l}\right)=\left(f\left(\sigma_{1}(t)\right), \cdots, f\left(\sigma_{k}(t)\right) ; f\left(y_{1}\right), \cdots, f\left(y_{l}\right)\right) .
\end{aligned}
$$

Recall that for a generic $(J, \nu)$ and for generic nonzero $t$, the image $\operatorname{Im}\left(\mathrm{e}_{\mathrm{t}}\right)$ intersects the product $\prod_{i=1}^{k} U_{i} \times \prod_{j=1}^{l} W_{j}$ transversally. Considering an intersection point $\left(f_{s} ; y_{s 1}, \cdots, y_{s l}\right)$, we can assign a sign to $\left(f_{s} ; y_{s 1}, \cdots, y_{s l}\right)$ by using the orientation of $\mathcal{M}_{A}^{v}\left(\Sigma_{t}, \mathcal{X}_{Z^{0}}, J, \nu_{t}\right),\left(\mathcal{X}_{Z^{0}}\right)^{k+l}$ and $\prod_{i=1}^{k} U_{i}^{0} \times \prod_{j=1}^{l} W_{j}^{0}$, where $Z^{0}, U_{i}^{0}$, and $W_{j}^{0}$ mean the top stratums. For $t=0$, after fix a position pattern $P_{1}$ of $\left(\sigma_{1}(0), \cdots, \sigma_{k}(0)\right)$ in $\Sigma_{0}$ and an intersection pattern $T$, again for generic $\left(J, \nu_{0}\right)$, the map $e_{0}$ intersects $\prod_{i=1}^{k} U_{i}^{0} \times \prod_{j=1}^{l} W_{j}^{0}$ transversally at finite many points in $\mathcal{X}_{Z^{0}}^{k+l}$. Let $\left(f_{0} ; y_{01}, \cdots, y_{0 l}\right)$ be one of such intersection points. Theorem 3.1 gives 
Corollary 3.2. Let $(J, \nu)$ be generic and $\left(f_{0} ; y_{01}, \cdots, y_{0 l}\right)$ be as the above which intersects transversally. Then there are $\epsilon, \delta>0$ such that for $t$ sufficiently small, there is a unique $\left(f_{t} ; y_{t 1}, \cdots, y_{t l}\right)$ in the space

$$
\mathcal{M}_{A}^{v}\left(\Sigma_{t}, \mathcal{X}_{Z^{0}}, J, \nu_{t}\right) \times\left(\Sigma_{t}\right)^{k+l}
$$

satisfying: (1) $d_{S}\left(y_{t j}, y_{0 j}\right) \leq \epsilon$, and $d_{\mathcal{X}_{z}}\left(f_{0}(x), f_{t}(y)\right) \leq \epsilon$ whenever $d_{S}(x, y) \leq \delta$. Moreover the sign associated to $\left(f_{t} ; y_{t 1}, \cdots, y_{t l}\right)$ is the same as the sign associated to $\left(f_{0} ; y_{01}, \cdots, y_{0 l}\right)$ for those generic $t$ such that $\mathcal{M}_{A}^{v}\left(\Sigma_{t}, \mathcal{X}_{Z^{0}}, J, \nu_{t}\right)$ is a smooth manifold.

In section 2 we have defined the fiberwise mixed invariants

$$
\Phi_{\left(A_{1}, \cdots, A_{m}, \omega, \Sigma_{0}, P\right)}^{v}\left(\alpha_{1}, \cdots, \alpha_{k} \mid \beta_{1}, \cdots, \beta_{l}\right)(\gamma)
$$

denote

$$
\Phi_{\left(A, \omega, \Sigma_{t}, P\right)}^{v}\left(\alpha_{1}, \cdots, \alpha_{k} \mid \beta_{1}, \cdots, \beta_{l}\right)(\gamma)
$$

by

$$
\Phi_{(A, \omega, g)}^{v}\left(\alpha_{1}, \cdots, \alpha_{k} \mid \beta_{1}, \cdots, \beta_{l}\right)(\gamma)
$$

where $P$ stands for the trivial position pattern and the trivial intersection pattern since $\Sigma_{t}$ is smooth.

Theorem 3.2 (Composition law). Suppose that fiber bundle $\mathcal{X} \rightarrow Y$ satisfies Assumption ( $\star$ ). Then for any rational cohomology classes

$$
\alpha_{1}, \cdots, \alpha_{k} ; \beta_{1}, \cdots, \beta_{j} \in H^{*}(\mathcal{X}, \mathbb{Q})
$$

and rational homology class $\gamma \in H_{*}(Y, \mathbb{Q})$,

$$
\begin{aligned}
& \Phi_{(A, \omega, g)}^{v}\left(\alpha_{1}, \cdots, \alpha_{k} \mid \beta_{1}, \cdots, \beta_{l}\right)(\gamma) \\
& =\sum_{A=A_{1}+\cdots A_{m}} \sum_{T} \Phi_{\left(A_{1}, \cdots, A_{m}, \omega, \Sigma_{0},\left(P_{1}, T\right)\right)}^{v}\left(\alpha_{1}, \cdots, \alpha_{k} \mid \beta_{1}, \cdots, \beta_{l}\right)(\gamma),
\end{aligned}
$$

for any fixed position pattern $P_{1}$.

Proof. Fix a degeneration $\pi: S \rightarrow \Delta$ of a $k$-point genus $g$ smooth stable curves, such that the central fiber is a stable curve $C$ and other fibers are smooth genus $g$ Riemann surfaces with $k$-marked points, where $\Delta \subset \mathbb{C}$ is an unit disc. Suppose that for $t \neq 0,\left(f_{t} ; y_{t 1}, \cdots, y_{t l}\right)$ is an intersection point, 
then $f_{t}\left(\sigma_{i}(t)\right) \in \operatorname{Im}\left(L_{i}\right)$, and $f_{t}\left(\Sigma_{t}\right) \cap \operatorname{Im}\left(M_{j}\right) \neq \emptyset$. By Proposition 1.1., and taking a subsequence if necessary, we may assume that $f_{t}$ converges to $f_{0}$ in $\overline{\mathcal{M}}_{\left(A_{1}, \cdots, A_{m}\right)}^{v}\left(\Sigma_{0}, \mathcal{X}_{Z}, J, \nu_{0}\right)$ for some partition $A_{1}, \cdots, A_{m}$ of $A$ and some intersection pattern $T$ as $t$ goes to zero. By Proposition 2.1, if $\left(J, \nu_{0}\right)$ is generic, $f_{0}$ is actually in $\mathcal{M}_{\left(A_{1}, \cdots, A_{m}\right)}^{v}\left(\Sigma_{0}, \mathcal{X}_{Z^{0}}, J, \nu_{0}\right)$, and by Proposition 1.5, we may assume that $f_{0}$ intersects transversally at every double points. Let $y_{j}^{0}$ be the limit of $y_{t j}$, clearly $f_{0}\left(x_{i}^{0}\right) \in \operatorname{Im}\left(L_{i}\right), f_{0}\left(y_{j}^{0}\right) \in \operatorname{Im}\left(M_{j}\right)$, therefore $\left(f_{0} ; y_{1}^{0}, \cdots, y_{l}^{0}\right)$ is an intersection point. Theorem follows from Corollary 3.2.

Next we want to show that $\Phi_{\left(A_{1}, \cdots, A_{m}, \omega, \Sigma_{0}, P\right)}^{v}\left(\alpha_{1}, \cdots, \alpha_{k} \mid \beta_{1}, \cdots, \beta_{l}\right)(\gamma)$ could be explicitly calculated in terms of the fiberwise mixed invariant of each component of $\Sigma_{0}$ and the contribution from the double points. There are two kinds of double points on a stable curve: intersection of two different components or self-intersection of one component. In each case we will give a formula.

Case 1. Suppose that $\Sigma_{0}$ has two components $\Sigma_{(01)}$ and $\Sigma_{(02)}$ of genus $g_{1}$ and $g_{2}$ respectively, satisfying: $(1) \Sigma_{(01)}$ and $\Sigma_{(02)}$ intersect at a double point $p ;(2) \Sigma_{(01)}$ contains $m$ marked points $x_{1}, \cdots, x_{m}$ and $\Sigma_{(02)}$ contains the rest of marked points. This is the position pattern $P_{1}$. Let $C=\left(\Sigma_{0} ; x_{1}, \cdots, x_{k}\right)$. Then $\Phi_{\left(A_{1}, A_{2}, \omega, C, P\right)}^{v}$ can be calculated as follows.

Assume that $Z$ is a pseudo-manifold representative of $\gamma$. Let $\left[H_{Z}^{\alpha}\right]$ be a basis of $H^{*}\left(\mathcal{X}_{Z}, \mathbb{Q}\right)$. Consider the diagonal $\Delta_{Z}$ in $\mathcal{X}_{Z} \times \mathcal{X}_{Z}$. By the Kunneth formula, we could write the Poincare dual of $\Delta_{Z}$ as $P D\left(\Delta_{Z}\right)=\sum_{\alpha, \beta} \eta_{(Z, \alpha, \beta)}$. $H_{Z}^{\alpha} \otimes H_{Z}^{\beta}$, where $\eta_{(Z, \alpha, \beta)}$ is the intersection matrix of $H^{*}\left(\mathcal{X}_{Z}, \mathbb{Q}\right)$.

Theorem 3.3. Let the intersection pattern $T$ be that the image of $\Sigma_{(01)}$ intersects $\operatorname{Im}\left(M_{j}\right), j=1, \cdots, l^{\prime}$ and that the image of $\Sigma_{(02)}$ intersects $\operatorname{Im}\left(M_{j}\right)$, $j=l^{\prime}+1, \cdots, l$. Let $A_{1}$ and $A_{2}$ be two homology classes in $H_{2}(V, \mathbb{Z})$. Then

$$
\begin{aligned}
& \Phi_{\left(A_{1}, A_{2}, \omega, C, P\right)}^{v}\left(\alpha_{1}, \cdots, \alpha_{k} \mid \beta_{1}, \cdots, \beta_{l}\right)(\gamma) \\
& =\sum_{\tau, \delta} \eta_{(Z, \tau, \delta)} \cdot \Phi_{\left(A_{1}, \omega, g_{1}\right)}^{v}\left(\alpha_{1}, \cdots, \alpha_{m},\left[H_{Z}^{\tau}\right] \mid \beta_{1}, \cdots, \beta_{l^{\prime}}\right)(\gamma) \\
& \quad \cdot \Phi_{\left(A_{2}, \omega, g_{2}\right)}^{v}\left(\alpha_{m+1}, \cdots, \alpha_{k},\left[H_{Z}^{\delta}\right] \mid \beta_{l^{\prime}+1}, \cdots, \beta_{l}\right)(\gamma)
\end{aligned}
$$


(3.50)

$$
\begin{aligned}
& \Phi_{(A, \omega, g)}^{v}\left(\alpha_{1}, \cdots, \alpha_{k} \mid \beta_{1}, \cdots, \beta_{l}\right)(\gamma) \\
& =\sum_{B_{1}+B_{2}=A} \sum_{j=1}^{l} \sum_{\sigma} \sum_{\tau, \delta} \frac{1}{j ! \cdot(l-j) !} \eta_{(Z, \tau, \delta)} \\
& \quad \cdot \Phi_{\left(A_{1}, \omega, g_{1}\right)}^{v}\left(\alpha_{1}, \cdots, \alpha_{m},\left[H_{Z}^{\tau}\right] \mid \beta_{\sigma(1)}, \cdots, \beta_{\sigma(j)}\right)(\gamma) \\
& \quad . \Phi_{\left(A_{2}, \omega, g_{2}\right)}^{v}\left(\alpha_{m+1}, \cdots, \alpha_{k},\left[H_{Z}^{\delta}\right] \mid \beta_{\sigma(j+1)}, \cdots, \beta_{\sigma(l)}\right)(\gamma),
\end{aligned}
$$

where $B_{1}$ and $B_{2}$ are any two homology classes in $H_{2}(V, \mathbb{Z})$ and $\sigma$ are permutations.

Proof. The proof follows from applying the argument of Theorem 7.3 in [RT] to moduli space $\mathcal{M}_{\left(A_{1}, A_{2}\right)}^{v}\left(\Sigma_{0}, \mathcal{X}_{Z}, J, \nu_{0}\right)$.

Case 2. Suppose that $\Sigma_{0}$ is a genus $g-1$ curve with one self-intersection point. Let $C=\left(\Sigma_{0} ; x_{1}, \cdots, x_{k}\right)$.

Theorem 3.4. We have

$$
\begin{aligned}
& \Phi_{(A, \omega, g)}^{v}\left(\alpha_{1}, \cdots, \alpha_{k} \mid \beta_{1}, \cdots, \beta_{l}\right)(\gamma) \\
& =\sum_{\tau, \delta} \eta_{(Z, \tau, \delta)} \cdot \Phi_{(A, \omega, g-1)}^{v}\left(\alpha_{1}, \cdots, \alpha_{k},\left[H_{Z}^{\tau}\right],\left[H_{Z}^{\delta}\right] \mid \beta_{1}, \cdots, \beta_{l}\right)(\gamma)
\end{aligned}
$$

Proof. The proof follows from applying the argument of Theorem 7.5 in [RT] to moduli space $\mathcal{M}_{A}^{v}\left(\Sigma_{0}, \mathcal{X}_{Z}, J, \nu_{0}\right)$.

\section{Fiberwise Quantum Cohomology and Equivariant Quantum Cohomology.}

Suppose that $\mathcal{X} \stackrel{p}{\rightarrow} Y$ is a fiber bundle with fiber $V$ satisfying Assumption $(\star)$. Let $A_{1}, \cdots, A_{m}$ be an integral basis of $H_{2}(V, \mathbb{Z})$. Any homology class $A \in H_{2}(V, \mathbb{Z})$ can then be written as $A=d_{1} \cdot A_{1}+\cdots+d_{m} \cdot A_{m}$. For simplicity we assume that for any genus zero $J$-holomorphic map $f$ of $V$, $f_{*}\left(\mathbb{C} P_{1}\right)=d_{1} \cdot A_{1}+\cdots+d_{m} \cdot A_{m}$ with $d_{i} \geq 0, i=1, \cdots, m$. 
Let

(4.1)

$$
\begin{aligned}
& \tilde{\Phi}_{\omega}^{v}\left(\alpha_{1}, \cdots, \alpha_{k}\right) \\
& =\sum_{A \in H_{2}(V,)} \Phi_{(A, \omega, 0)}^{v}\left(\alpha_{1}, \cdots, \alpha_{k} \mid\right)([Y]) q_{1}^{d_{1}} \cdots q_{m}^{d_{m}} \quad \in \mathbb{C}\left[\left[q_{1}, \cdots, q_{m}\right]\right],
\end{aligned}
$$

where $\alpha_{i} \in H^{*}(\mathcal{X}, \mathbb{C}), i=1, \cdots, k, q_{1}, \cdots, q_{m}$ are parameters, and $\mathbb{C}\left[\left[q_{1}, \cdots, q_{m}\right]\right]$ is the set of all formal power series of $q_{i}$.

We define fiberwise quantum multiplication

$$
\begin{aligned}
*: & H^{*}(\mathcal{X}, \mathbb{C})\left[\left[q_{1}, \cdots, q_{m}\right]\right] \times H^{*}(\mathcal{X}, \mathbb{C})\left[\left[q_{1}, \cdots, q_{m}\right]\right] \rightarrow \\
& H^{*}(\mathcal{X}, \mathbb{C})\left[\left[q_{1}, \cdots, q_{m}\right]\right]
\end{aligned}
$$

by

$$
\alpha * \beta(B)=\tilde{\Phi}_{\omega}^{v}(\alpha, \beta, P D(B)),
$$

where $\alpha, \beta \in H^{*}(\mathcal{X}, \mathbb{C}), B \in H_{*}(\mathcal{X}, \mathbb{C})$, and $P D(B)$ is the Poincare dual of $B$. We extend $*$ linearly to all of $H^{*}(\mathcal{X}, \mathbb{C})\left[\left[q_{1}, \cdots, q_{m}\right]\right]$.

We call $H^{*}(\mathcal{X}, \mathbb{C})\left[\left[q_{1}, \cdots, q_{m}\right]\right]$ with fiberwise quantum multiplication * the fiberwise quantum cohomology associated to the fiber bundle $\mathcal{X} \stackrel{p}{\rightarrow}$ $Y$, denoted by $Q H^{*}(\mathcal{X}, Y, \mathbb{C})$. Note that when $Y$ is a point, the fiberwise quantum cohomology becomes quantum cohomology of $V$.

Theorem 4.1. For any fiber bundle $\mathcal{X} \stackrel{p}{\rightarrow} Y$ with fiber $V_{1}$ and fiberwise symplectic form $\omega$ satisfying Assumption ( $*$ ), there is a well defined fiberwise quantum cohomology. It has the following properties.

(i) It is a $H^{*}(Y, \mathbb{C})\left[\left[q_{1}, \cdots, q_{m}\right]\right]$ module. This module structure is the same as that of $H^{*}(\mathcal{X}, \mathbb{C})$ as a $H^{*}(Y, \mathbb{C})$-module.

(ii) It is graded commutative:

$$
\alpha * \beta=(-1)^{\operatorname{deg} \alpha \cdot \operatorname{deg} \beta} \cdot \beta * \alpha .
$$

(iii) (Direct Product) For any two fiber bundles $\mathcal{X}_{1} \rightarrow Y_{1}$ and $\mathcal{X}_{2} \rightarrow Y_{2}$ satisfying Assumption ( $\star$ ). There is an isomorphism

$$
Q H^{*}\left(\mathcal{X}_{1} \times \mathcal{X}_{2}, Y_{1} \times Y_{2}, \mathbb{C}\right) \cong Q H^{*}\left(\mathcal{X}_{1}, Y_{1}, \mathbb{C}\right) \times Q H^{*}\left(\mathcal{X}_{2}, Y_{2}, \mathbb{C}\right)
$$

as a $H^{*}\left(Y_{1} \times Y_{2}, \mathbb{C}\right)\left[\left[q_{1}, \cdots, q_{m_{1}+m_{2}}\right]\right]-$ module. Here $q_{1}, \cdots, q_{m_{1}}$ are parameters for $Q H^{*}\left(\mathcal{X}_{1}, Y_{1}, \mathbb{C}\right)$ and $q_{m_{1}+1}, \cdots, q_{m_{1}+m_{2}}$ are the parameters for $Q H^{*}\left(\mathcal{X}_{2}, Y_{2}, \mathbb{C}\right)$. 
(iv) (Restriction) Let $Y_{1}$ be a closed finite dimensional manifold and $h$ : $Y_{1} \rightarrow Y$ be a smooth map. Set $\mathcal{X}_{Y_{1}}=\mathcal{X} \times_{h} Y_{1}$, the induced fiber bundle with fiber $V$ and induced fiberwise symplectic form $\omega$. Then there are a natural map $H: \mathcal{X}_{Y_{1}} \rightarrow \mathcal{X}$ and a $H^{*}(Y, \mathbb{C})\left[\left[q_{1}, \cdots, q_{m}\right]\right]-$ module homomorphism

$$
H^{*}: Q H^{*}(\mathcal{X}, Y, \mathbb{C}) \rightarrow Q H^{*}\left(\mathcal{X}_{Y_{1}}, Y_{1}, \mathbb{C}\right)
$$

Here we assume that $Q H^{*}\left(\mathcal{X}_{Y_{1}}, Y_{1}, \mathbb{C}\right)$ has the induced $H^{*}(Y, \mathbb{C})\left[\left[q_{1}, \cdots, q_{m}\right]\right]-$ module structure from $h^{*}: H^{*}(Y, \mathbb{C}) \rightarrow H^{*}\left(Y_{1}, \mathbb{C}\right)$.

(v) (Induction) Suppose that fiber bundle $Y \rightarrow B$ has fiber $V_{2}$ with fiberwise symplectic form $\omega_{2}$ satisfying Assumption ( $\star$ ). Assume that there is another fiberwise symplectic form $\omega$ satisfying Assumption (*) for the induced fiber bundle $\mathcal{X} \rightarrow B$ with fiber $V$. Assume further that $H_{2}(V, \mathbb{Z})$ has an integral basis $B_{1}, \cdots, B_{m_{1}+m_{2}}$ such that $B_{m_{1}+1}, \cdots, B_{m_{1}+m_{2}}$ are the integral basis of $H_{2}\left(V_{2}, \mathbb{Z}\right)$. Then there is an isomorphism

$$
\begin{aligned}
& Q H^{*}(\mathcal{X}, B, \mathbb{C}) /\left\{\left(q_{m_{1}+1}, \cdots, q_{m_{1}+m_{2}}\right) \cdot H^{*}(\mathcal{X}, B, \mathbb{C})\right\} \cong Q H^{*}(\mathcal{X}, Y, \mathbb{C}), \\
& \text { as a } H^{*}(B, \mathbb{C})\left[\left[q_{1}, \cdots, q_{m_{1}}\right]\right]-\text { module. }
\end{aligned}
$$

Proof. The proof follows directly from the corresponding properties of the fiberwise mixed invariants.

Theorem 4.2. For any fiber bundle $\mathcal{X} \stackrel{p}{\rightarrow} Y$ satisfying Assumption $(\star)$, fiberwise quantum cohomology $Q H^{*}(\mathcal{X}, Y, \mathbb{C})$ is associative.

Proof. Suppose $\alpha_{1}, \alpha_{2}, \alpha_{3} \in H^{*}(\mathcal{X}, \mathbb{C}), H^{a}$ is a basis of $H^{*}(\mathcal{X}, \mathbb{C})$ and $\left(\eta_{a, b}\right)$ is the intersection matrix, then

$$
\begin{aligned}
& \left(\alpha_{1} * \alpha_{2}\right) * \alpha_{3} \\
& =\left(\eta_{a_{1}, b_{1}} \cdot \tilde{\Phi}_{\omega}^{v}\left(\alpha_{1}, \alpha_{2}, H^{a_{1}}\right) \cdot H^{b_{1}}\right) * \alpha_{3} \\
& =\eta_{a_{1}, b_{1}} \cdot \tilde{\Phi}_{\omega}^{v}\left(\alpha_{1}, \alpha_{2}, H^{a_{1}}\right) \cdot \tilde{\Phi}_{\omega}^{v}\left(H^{b_{1}}, \alpha_{3}, H^{a_{2}}\right) \cdot \eta_{a_{2}, b_{2}} \cdot H^{b_{2}}
\end{aligned}
$$


and

$$
\begin{aligned}
& \alpha_{1} *\left(\alpha_{2} * \alpha_{3}\right) \\
& =\alpha_{1} *\left(\eta_{a_{1}, b_{1}} \cdot \tilde{\Phi}_{\omega}^{v}\left(\alpha_{2}, \alpha_{3}, H^{a_{1}}\right) \cdot H^{b_{1}}\right) \\
& =\eta_{a_{1}, b_{1}} \cdot \tilde{\Phi}_{\omega}^{v}\left(\alpha_{1}, H^{b_{1}}, H^{a_{2}}\right) \cdot \tilde{\Phi}_{\omega}^{v}\left(\alpha_{2}, \alpha_{3}, H^{a_{1}}\right) \cdot \eta_{a_{2}, b_{2}} \cdot H^{b_{2}} .
\end{aligned}
$$

It follows from Theorem 3.3 that

$$
\begin{aligned}
& \eta_{a_{1}, b_{1}} \cdot \tilde{\Phi}_{\omega}^{v}\left(\alpha_{1}, \alpha_{2}, H^{a_{1}}\right) \cdot \tilde{\Phi}_{\omega}^{v}\left(H^{b_{1}}, \alpha_{3}, H^{a_{2}}\right) \\
= & \tilde{\Phi}_{\omega}^{v}\left(\alpha_{1}, \alpha_{2}, \alpha_{3}, H^{a_{2}}\right) \\
& =\eta_{a_{1}, b_{1}} \cdot \tilde{\Phi}_{\omega}^{v}\left(\alpha_{1}, H^{b_{1}}, H^{a_{2}}\right) \cdot \tilde{\Phi}_{\omega}^{v}\left(\alpha_{2}, \alpha_{3}, H^{a_{1}}\right) .
\end{aligned}
$$

So

$$
\left(\alpha_{1} * \alpha_{2}\right) * \alpha_{3}=\alpha_{1} *\left(\alpha_{2} * \alpha_{3}\right)
$$

The theorem is proved.

Suppose that Lie group $G$ acts on a semi-positive symplectic manifold $(V, \omega)$, keeping $\omega$ invariant. Denote by $E G \rightarrow B G$ the universal $G$ bundle. Let $Y=B G$ and $\mathcal{X}=V \times_{G} E G$. However we can not apply the fiberwise quantum multiplication directly to this case because $Y$ is infinite dimensional. Note that by the definition equivariant cohomology $H_{G}^{*}(V, \mathbb{C})=H^{*}(\mathcal{X}, \mathbb{C})$. To define the equivariant quantum multiplication $\alpha * \beta$ for $\alpha$ and $\beta \in H_{G}^{*}(V, \mathbb{C})$, we use finite dimensional approximation of $Y$. We choose a $N$ dimensional approximation $B G_{N}$ of $B G$ such that $H^{i}\left(B G_{N}, \mathbb{C}\right)$ are isomorphic to $H^{i}(B G, \mathbb{C})$ for $i \leq \operatorname{deg} \alpha+\operatorname{deg} \beta+1$. Then $\mathcal{X}_{N}=V \times_{G} B G_{N}$ has same cohomology as $\mathcal{X}$ up to degree at least $\operatorname{deg} \alpha+\operatorname{deg} \beta+1$. We define $\alpha * \beta$ to be $(\alpha * \beta)_{N}$, defined using $\mathcal{X}_{N} \rightarrow B G_{N}$.

We need to show this multiplication is well-defined. Suppose $N^{\prime}$ is another such integer. We need to show $(\alpha * \beta)_{N}(B)=(\alpha * \beta)_{N^{\prime}}(B)$, for any $B \in H_{*}(\mathcal{X}, \mathbb{C})$. Since

$$
\begin{aligned}
& (\alpha * \beta)_{N}(B)=\sum_{A \in H_{2}(V,)} \Phi_{(A, \omega, 0)}^{\mathcal{X}_{N}, v}(\alpha, \beta, P D(B))\left(\left[B G_{N}\right]\right) q_{1}^{d_{1}} \cdots q_{m}^{d_{m}} \\
& (\alpha * \beta)_{N^{\prime}}(B)=\sum_{A \in H_{2}(V,)} \Phi_{(A, \omega, 0)}^{\mathcal{X}_{N^{\prime}, v}}(\alpha, \beta, P D(B))\left(\left[B G_{N^{\prime}}\right]\right) q_{1}^{d_{1}} \cdots q_{m}^{d_{m}}
\end{aligned}
$$

where $\Phi_{(A, \omega, 0)}^{\mathcal{X}_{N}, v}$ and $\Phi_{(A, \omega, 0)}^{\mathcal{X}_{N^{\prime}, v}}$ are the fiberwise mixed invariants defined by $\mathcal{X}_{N}$ and $\mathcal{X}_{N^{\prime}}$ respectively. So all we need to show is

$$
\Phi_{(A, \omega, 0)}^{\mathcal{X}_{N}, v}(\alpha, \beta, P D(B))\left(\left[B G_{N}\right]\right)=\Phi_{(A, \omega, 0)}^{\mathcal{X}_{N^{\prime}}, v}(\alpha, \beta, P D(B))\left(\left[B G N^{\prime}\right]\right) .
$$


Without loss of generality, assume that $N<N^{\prime}$. Choosing $B G_{N} \subset$ $B G_{N^{\prime}} \subset B G$ to be submanifold, then $\mathcal{X}_{N} \subset \mathcal{X}_{N^{\prime}}$ is also a submanifold. From the choice of $N$, we can choose pseudo-manifold representatives $\left(L_{1}, U_{1}\right),\left(L_{2}, U_{2}\right)$ of $\alpha, \beta$ in $\mathcal{X}_{N^{\prime}}$ such that their images, intersecting with $\mathcal{X}_{N}$, give the pseudo-manifold representatives of $\alpha$ and $\beta$ in $\mathcal{X}_{N}$. Let $\left(L_{3}, U_{3}\right)$ be the pseudo-manifold representative of $B$ in $\mathcal{X}_{N}$. Under the inclusion $\mathcal{X}_{N} \subset \mathcal{X}_{N^{\prime}}$, we view $\left(L_{3}, U_{3}\right)$ as a representative of $B$ in $\mathcal{X}_{N^{\prime}}$. From Remark 1.4 (i), we know that there is a generic $(J, \nu)$ which is good for $\mathcal{X}_{N^{\prime}}$ and its restriction to $\mathcal{X}_{N}$ is also good. $\Phi_{(A, \omega, 0)}^{\mathcal{X}_{N^{\prime}, v}}(\alpha, \beta, \delta)\left(B G_{N^{\prime}}\right)$ is defined as counting the number of the intersection of $\mathcal{M}_{A}^{v}\left(S^{2}, \mathcal{X}_{N^{\prime}}, J, \nu\right) \times\left(S^{2}\right)^{3}$ with $\left(L_{1}, U_{1}\right),\left(L_{2}, U_{2}\right),\left(L_{3}, U_{3}\right)$ under evaluation map. The intersections happen only inside $\mathcal{X}_{N}$ because of the choice of $\left(L_{3}, U_{3}\right)$. Also note that $\mathcal{M}_{A}^{v}\left(S^{2}, \mathcal{X}_{N}, J, \nu\right)$ is a subspace of $\mathcal{M}_{A}^{v}\left(S^{2}, \mathcal{X}_{N^{\prime}}, J, \nu\right)$. So the intersection are exactly the same as the intersections of $\mathcal{M}_{A}^{v}\left(S^{2}, \mathcal{X}_{N}, J, \nu\right) \times\left(S^{2}\right)^{3}$ with $\left(L_{1}, U_{1}\right),\left(L_{2}, U_{2}\right),\left(L_{3}, U_{3}\right)$ under evaluation map. The number of the latter intersections is by definition $\Phi_{\left(A, \omega, S^{2}\right)}^{\mathcal{X}_{N}, v}(\alpha, \beta, P D(B))\left(B G_{N}\right)$. So welldefinedness is proved.

When $Y$ is infinite dimensional, for a fiber bundle $\mathcal{X} \rightarrow Y$ satisfying Assumption ( $\star$ ), we can define the fiberwise quantum cohomology $Q H^{*}(\mathcal{X}, Y, \mathbb{C})$ similarly. We will use this definition in section 5.2.

We call $H_{G}^{*}(V, \mathbb{C})\left[\left[q_{1}, \cdots, q_{m}\right]\right]$ with equivariant quantum multiplication equivariant quantum cohomology, denoted by $Q H_{G}^{*}(V, \mathbb{C})$. It is a $q$ deformation of equivariant cohomology. The following two theorems follow from Theorem 4.1 and 4.2 .

Theorem 4.3. Suppose that Lie group $G$ acts on a strong semi-positive symplectic manifold $(V, \omega)$, keeping $\omega$ invariant. Then there is a well-defined equivariant quantum cohomology $Q H_{G}^{*}(V, \mathbb{C})$. It has the following properties.

(i) $Q H_{G}^{*}(V, \mathbb{C})$ is a $H^{*}(B G, \mathbb{C})\left[\left[q_{1}, \cdots, q_{m}\right]\right]-$ module. This module structure is the same as the $H^{*}(B G ; \mathbb{C})$-module structure of $H_{G}^{*}(V ; \mathbb{C})$.

(ii) $Q H_{G}^{*}(V, \mathbb{C})$ is graded commutative:

$$
\alpha * \beta=(-1)^{\operatorname{deg} \alpha+\operatorname{deg} \beta} \beta * \alpha .
$$

(iii) (Direct Product) Suppose that Lie groups $G_{i}$ act on two strong semipositive symplectic manifolds $\left(V_{i}, \omega_{i}\right)$, keeping $\omega_{i}$ invariant, $i=1$, 2 . Then

$$
Q H_{G_{1} \times G_{2}}^{*}\left(V_{1} \times V_{2}, \mathbb{C}\right) \cong Q H_{G_{1}}^{*}\left(V_{1}, \mathbb{C}\right) \otimes Q H_{G_{2}}^{*}\left(V_{2}, \mathbb{C}\right),
$$


where $\omega_{1} \times \omega_{2}$ is the symplectic form on $V_{1} \times V_{2}$ and $G_{1} \times G_{2}$ acts on $V_{1} \times V_{2}$ by product action.

(iv) (Restriction) Suppose $\tilde{G} \subset G$ is a normal Lie subgroup. Then there is a homomorphism

$$
H^{*}: Q H_{G}^{*}(V, \mathbb{C}) \rightarrow Q H_{\tilde{G}}^{*}(V, \mathbb{C})
$$

as a $H^{*}(B G)\left[\left[q_{1}, \cdots, q_{m}\right]\right]-$ module. Here we choose $E \tilde{G}=E G$ which induces a map $B \tilde{G} \rightarrow B G$ and the $H^{*}(B G)\left[\left[q_{1}, \cdots, q_{m}\right]\right]$-module structure of $Q H_{\tilde{G}}^{*}(V, \mathbb{C})$ is induced from $h^{*}: H^{*}(B G, \mathbb{C}) \rightarrow H^{*}(B \tilde{G}, \mathbb{C})$.

(v) (Induction) Let $\tilde{G} \subset G$ be a normal Lie subgroup with $G / \tilde{G}$ being simply connected. Suppose that $G / \tilde{G}$ is a strong semi-positive symplectic manifold. Assume that $\tilde{G}$ acts on a strong semi-positive symplectic manifold $(\tilde{V}, \tilde{\omega})$, keeping $\tilde{\omega}$ invariant. Let $V=\tilde{V} \times_{\tilde{G}} G$. Assume that $\tilde{\omega}$ can be extended to a strong semi-positive symplectic form on $V$ invariant under $G$ action. Assume further that $H_{2}(V, \mathbb{Z})$ has basis $B_{1}, \cdots, B_{m_{1}+m_{2}}$ with $B_{m_{1}+1}, \cdots, B_{m_{1}+m_{2}}$ being basis of $G / \tilde{G}$. Then

$$
Q H_{G}^{*}(V ; \mathbb{C}) /\left\{\left(q_{m_{1}+1}, \cdots, q_{m_{1}+m_{2}}\right) Q H_{G}^{*}(V, \mathbb{C})\right\} \cong Q H_{\tilde{G}}^{*}(\tilde{V}, \mathbb{C}) .
$$

Theorem 4.4. Equivariant quantum cohomology is associative.

\section{Several Examples.}

\subsection{Classical cohomology ring.}

Let $n$ be a natural number and $k_{1}, \cdots, k_{s}$ be a partition of $n$, i.e., $k_{1}+$ $\cdots+k_{s}=n\left(k_{1}, \cdots, k_{s}\right.$ are natural numbers). Recall that a partial flag manifold $F_{\left(k_{1}, \cdots, k_{s}\right)}$ is the set of all flags in $\mathbb{C}^{n}, 0 \subset C_{1} \subset \cdots \subset C_{s}=\mathbb{C}^{n}$ with $\operatorname{dim} C_{i}=k_{1}+\cdots+k_{i}, i=1, \cdots, s$. There are complex vector bundles $L_{i}$ of rank $k_{i}$ over $F_{\left(k_{1}, \cdots, k_{s}\right)}$ whose fiber at above flag is $C_{i} / C_{i-1}$. Here we use convention $C_{0}=0$. Denote by $\mathbb{C}^{n}$ the trivial bundle of rank $n$. These bundles satisfy the following relation

$$
L_{1} \oplus \cdots \oplus L_{s}=\mathbb{C}^{n} .
$$


Lemma 5.1. The cohomology ring $H^{*}\left(F_{\left(k_{1}, \cdots, k_{s}\right)}, \mathbb{C}\right)$ is isomorphic to

$$
\mathbb{C}\left[u_{1}^{1}, \cdots, u_{k_{1}}^{1} ; \cdots ; u_{1}^{s}, \cdots, u_{k_{s}}^{s}\right] /\left\{\sigma_{1}, \cdots, \sigma_{n}\right\},
$$

where $u_{j}^{i}$ are parameters which we assign with a degree $2 j$, and $\sigma_{i}$ is degree $2 i$ part of the expansion of $\left(1+u_{1}^{1}+\cdots, u_{k_{1}}^{1}\right) \cdots\left(1+u_{1}^{s}+\cdots+u_{k_{s}}^{s}\right)$. Under the isomorphism $u_{j}^{i}$ is mapped to the $j$-th Chern class $c_{j}\left(L_{i}\right)$. Furthermore the first Chern class of $F_{\left(k_{1}, \cdots, k_{s}\right)}$ is $\sum_{j=1}^{s-1}\left(k_{j}+k_{j+1}\right) p_{j}$ where $p_{j}=\sum_{k=j}^{s-1} u_{1}^{k+1}$.

Proof. This result is well-known. For convenience, we sketch a proof.

Because of (5.1), there is a natural map which maps $u_{j}^{i}$ to $c_{j}\left(L_{i}\right)$,

$$
\mathbb{C}\left[u_{1}^{1}, \cdots, u_{k_{1}}^{1} ; \cdots ; u_{1}^{s}, \cdots, u_{k_{s}}^{s}\right] /\left\{\sigma_{1}, \cdots, \sigma_{n}\right\} \stackrel{p}{\rightarrow} H^{*}\left(F_{\left(k_{1}, \cdots, k_{s}\right)}, \mathbb{C}\right) .
$$

First we show that $p$ is surjective by showing that $H^{*}\left(F_{\left(k_{1}, \cdots, k_{s}\right)} ; \mathbb{C}\right)$ is generated by $c_{j}\left(L_{i}\right)$. We argue by induction. When $s=2, F_{k_{1}, k_{2}}$ is a Grassmannian. The statement is true. Suppose that it is true for $s-1$ case. Then consider fiberation

$$
F_{\left(k_{1}, \cdots, k_{s}\right)} \stackrel{\pi}{\rightarrow} G\left(n-k_{s}, n\right),
$$

with a fiber $F_{\left(k_{1}, \cdots, k_{s-1}\right)}\left(\mathbb{C}^{n-k_{s}}\right)$ where $\pi$ maps flag $0 \subset C_{1} \subset \cdots \subset C_{s}$ to $C_{s-1}$. Note it is easily to see the restriction of $c_{j}\left(L_{i}\right)$ for $1 \leq i \leq s-1$ to each fiber is the Chern class of those " $L_{i}$ "s of the fiber, by assumption they generate the cohomology of the fiber. The Chern classes of similarly constructed rank $k_{s}$ bundle $\tilde{L}_{2}$ on $G\left(n-k_{s}, n\right)$ generate the cohomology of $G\left(n-k_{s}, n\right)$, and they are mapped to $c_{j}^{s}$ under $\pi^{*}$. So the Leray-Hirsch Theorem for fibration applies. $H^{*}\left(F_{\left(k_{1}, \cdots, k_{s}\right)}, \mathbb{C}\right)$ is generated by $c_{j}\left(L_{i}\right)$ 's.

Secondly, we show that $p$ is an isomorphism. The Poincare series $P_{t}\left(H^{*}\left(F_{\left(k_{1}, \cdots, k_{s}\right)}, \mathbb{C}\right)\right)$ is the product of Poincare series of $H^{*}\left(F_{\left(k_{1}, \cdots, k_{s-1}\right)}, \mathbb{C}\right)$ and $H^{*}\left(G\left(n-k_{s}, n\right), \mathbb{C}\right)$, a simple induction gives

$$
P_{t}\left(H^{*}\left(F_{\left(k_{1}, \cdots, k_{s}\right)}, \mathbb{C}\right)\right)=\frac{\prod_{i=1}^{n}\left(1-t^{2 i}\right)}{\prod_{j=1}^{s} \prod_{i=1}^{k_{j}}\left(1-t^{2 i}\right)},
$$

which is exactly the Poincare series of

$$
\mathbb{C}\left[u_{1}^{1}, \cdots, u_{k_{1}}^{1} ; \cdots ; u_{1}^{s}, \cdots, u_{k_{s}}^{s}\right] /\left\{\sigma_{1}, \cdots, \sigma_{n}\right\}
$$

(see [BT], section 23). So $p$ is an isomorphism. 
Denote by $W_{j}$ the vector bundle of rank $\sum_{i=j}^{s-1} k_{i+1}$ over $F_{\left(k_{1}, \cdots, k_{s}\right)}$ with fiber at flag $0 \subset C_{1} \subset \cdots \subset C_{s}$ to be $\mathbb{C}^{n} / C_{j}$. Note that $W_{j} \cong \oplus_{i=j}^{s-1} L_{i+1}$ and

$$
T F_{\left(k_{1}, \cdots, k_{s}\right)}=\oplus_{j=1}^{s-1} \operatorname{Hom}\left(L_{j}, W_{j}\right) .
$$

It follows

$$
\begin{aligned}
c_{1}\left(F_{\left(k_{1}, \cdots, k_{s}\right)}\right) & =\sum_{j=1}^{s-1} c_{1}\left(\operatorname{Hom}\left(L_{j}, W_{j}\right)\right) \\
& =\sum_{j=1}^{s-1} c_{1}\left(L_{j}^{*} \otimes W_{j}\right) \\
& =\sum_{j=1}^{s-1}\left[-\left(k_{j+1}+\cdots+k_{s}\right) c_{1}\left(L_{j}\right)+k_{j} c_{1}\left(W_{j}\right)\right] \\
& =\sum_{j=1}^{s-1}\left[-\left(k_{j+1}+\cdots+k_{s}\right) u_{1}^{j}+k_{j}\left(u_{1}^{j+1}+\cdots+u_{1}^{s}\right)\right] \\
& =k_{2} p_{1}+\cdots+k_{s} p_{s-1}+k_{1} p_{1}+\cdots+k_{s-1} p_{s-1} \\
& =\sum_{j=1}^{s-1}\left(k_{j}+k_{j+1}\right) p_{j} .
\end{aligned}
$$

Where we use the fact $-\left(u_{1}^{1}+\cdots+u_{1}^{j}\right)=p_{j}$ in the second to last step. This fact follows from $u_{1}^{1}+\cdots+u_{1}^{s}=0$.

Suppose that $E \rightarrow X$ is a complex vector bundle of rank $n$. The partial flag manifold $F_{\left(k_{1}, \cdots, k_{s}\right)}(E)$ over $X$ can be constructed as follows. Let $\left\{U_{\alpha}\right\}$ be a covering of $X$ such that $E$ can be trivialized on each $U_{\alpha},\left.E\right|_{U_{\alpha}} \cong$ $U_{\alpha} \times \mathbb{C}^{n}$, with transition functions $\left(\phi_{\alpha \beta}, A_{\alpha \beta}\right)$, where $\phi_{\alpha \beta}: U_{\alpha} \rightarrow U_{\beta}$ and $A_{\alpha \beta}: U_{\alpha} \cap U_{\beta} \rightarrow G L(n, \mathbb{C})$. Then patching together $U_{\alpha} \times F_{\left(k_{1}, \cdots, k_{s}\right)}$ using the induced action of $A_{\alpha \beta}$ on $F_{\left(k_{1}, \cdots, k_{s}\right)}$, we get $F_{\left(k_{1}, \cdots, k_{s}\right)}(E)$. Note that $F_{\left(k_{1}, \cdots, k_{s}\right)}(E) \stackrel{p}{\rightarrow} X$ is a fiber bundle with fiber $F_{\left(k_{1}, \cdots, k_{s}\right)}$. Similarly we can define vector bundle $L_{i}(E)$ over $F_{\left(k_{1}, \cdots, k_{s}\right)}(E)$. It is not hard to see

$$
L_{1}(E) \oplus \cdots L_{s}(E) \cong p^{*} E .
$$

Lemma 5.2. The cohomology ring $H^{*}\left(F_{\left(k_{1}, \cdots, k_{s}\right)}(E), \mathbb{C}\right)$ is isomorphic to $H^{*}(X ; \mathbb{C})\left[u_{1}^{1}, \cdots, u_{k_{1}}^{1} ; \cdots ; u_{1}^{s}, \cdots, u_{k_{s}}^{s}\right] /\left\{\sigma_{1}-c_{1}\left(p^{*} E\right), \cdots, \sigma_{n}-c_{n}\left(p^{*} E\right)\right\}$, 
as a $H^{*}(X, \mathbb{C})$-module. Here parameter $u_{j}^{i}$ is mapped to $c_{j}\left(L_{i}(E)\right)$ and $\sigma_{j}$ is defined as in Lemma 5.1.

Proof. Consider fiberation $F_{\left(k_{1}, \cdots, k_{s}\right)}(E) \stackrel{p}{\rightarrow} X$ which is a fiber bundle with fiber $F_{\left(k_{1}, \cdots, k_{s}\right)}$. Note that the restriction of $c_{j}\left(L_{i}(E)\right)$ to each fiber $F_{\left(k_{1}, \cdots, k_{s}\right)}$ gives $c_{j}\left(L_{i}\right)$. The lemma follows from the Leray-Hirsch theorem for fiberation and (5.3).

Note that the standard action of $U(n)$ on $\mathbb{C}^{n}$ induces an action of $U(n)$ on $F_{\left(k_{1}, \cdots, k_{s}\right)}$ and a fiberwise action on $F_{\left(k_{1}, \cdots, k_{s}\right)}(E)$. So we can make the following construction. Suppose $G=U\left(l_{1}\right) \times \cdots \times U\left(l_{t}\right) \subset U(n)$ is a subgroup with the induced action on $F_{\left(k_{1}, \cdots, k_{s}\right)}$ and $F_{\left(k_{1}, \cdots, k_{s}\right)}(E)$. Then

$$
F_{\left(k_{1}, \cdots, k_{s}\right)}(E) \times_{G} E G \stackrel{p}{\rightarrow} X \times B G
$$

is a fiber bundle with a fiber $F_{\left(k_{1}, \cdots, k_{s}\right)}$. Here $E G \rightarrow B G$ is the universal principal $G$ bundle. Construct the $L_{i}(E, G)$ bundle over $F_{\left(k_{1}, \cdots, k_{s}\right)}(E) \times_{G} E G$ as before. There is a bundle $\tilde{E}=E \times_{U(n)} E G$ of rank $n$ over $X \times B G$ whose restriction to each $X$ slice is $E$ and whose restriction to each $B G$ slice is $E G \times{ }_{G} \mathbb{C}^{n}$. Denote by $c_{i}(G)$ the i-th Chern class of universal $G$ bundle $E G \times{ }_{G} \mathbb{C}^{n}$. Then $c_{i}(\tilde{E})$ can be expressed in terms of $c_{j}(E)$ and $c_{k}(G)$, $i, j, k=1, \cdots, n$, and

$$
L_{1}(E, G) \oplus \cdots \oplus L_{s}(E, G) \cong p^{*} \tilde{E}
$$

Lemma 5.3. The cohomology ring $H^{*}\left(F_{\left(k_{1}, \cdots, k_{s}\right)}(E) \times_{G} E G, \mathbb{C}\right)$ is isomorphic to

$$
\begin{aligned}
& H^{*}(X, \mathbb{C}) \times H^{*}(B G, \mathbb{C})\left[u_{1}^{1}, \cdots, u_{k_{1}}^{1} ; \cdots ; u_{1}^{s}, \cdots, u_{k_{s}}^{s}\right] \\
& \quad /\left\{\sigma_{1}-c_{1}\left(p^{*} \tilde{E}\right), \cdots, \sigma_{n}-c_{n}\left(p^{*} \tilde{E}\right)\right\}
\end{aligned}
$$

as a $H^{*}(X, \mathbb{C}) \times H^{*}(B G, \mathbb{C})$-module. Here parameter $u_{j}^{i}$ is mapped to $c_{j}\left(L_{i}(E, G)\right)$, and $\sigma_{j}$ is defined as in Lemma 5.1.

Proof. The proof, which is similar to the proof of lemma 5.2, is omitted. 
Note that if $X$ is a point and $G=U(n)$ in Lemma 5.3, we get the equivariant cohomology of flag manifold

$$
\begin{aligned}
& H_{U(n)}^{*}\left(F_{\left(k_{1}, \cdots, k_{s}\right)}, \mathbb{C}\right) \\
& =C\left[u_{1}^{1}, \cdots, u_{k_{1}}^{1} ; \cdots ; u_{1}^{s}, \cdots, u_{k_{s}}^{s} ; c_{1}(U(n)), \cdots, c_{n}(U(n))\right] \\
& \quad /\left\{\sigma_{1}-c_{1}(U(n)), \cdots, \sigma_{n}-c_{n}(U(n))\right\} .
\end{aligned}
$$

Two special cases are:

Case 1. Consider the diagonal embedding $U(l) \rightarrow U(l) \times \cdots \times U(l) \subset U(n)$, $t$ factors $U(l)$. This embedding induces an action of $U(l)$ on $\mathbb{C}^{n}$; denote this action by $\rho_{l}$. Then the cohomology ring $H^{*}\left(F_{\left(k_{1}, \cdots, k_{s}\right)} \times_{\rho_{l}} E U(l), \mathbb{C}\right)$ is isomorphic to

$$
\begin{gathered}
\mathbb{C}\left[u_{1}^{1}, \cdots, u_{k_{1}}^{1} ; \cdots ; u_{1}^{s}, \cdots, u_{k_{s}}^{s} ; c_{1}(U(l)), \cdots, c_{l}(U(l))\right] \\
\quad /\left\{\sigma_{1}-\tilde{c}_{1}, \cdots, \sigma_{n}-\tilde{c}_{n}\right\}
\end{gathered}
$$

where $u_{j}^{i}$ and $\sigma_{j}$ are the same as in Lemma 5.1 and $\tilde{c_{j}}$ is given by $1+\tilde{c}_{1}+$ $\cdots+\tilde{c}_{n}=\left[1+c_{1}(U(l))+\cdots+c_{l}(U(l))\right]^{t}$.

Case 2. Consider an embedding $U(1) \rightarrow U(1) \times \cdots \times U(1)$ which send $e^{2 \pi \sqrt{-1} \theta}$ to $\left(e^{2 \pi \sqrt{-1} r_{1} \theta}, \cdots, e^{2 \pi \sqrt{-1} r_{n} \theta}\right)$ with $r_{i} \in \mathbb{Z}$. This embedding induces an action of $U(1)$ on $\mathbb{C}^{n}$; denote this action by $\tilde{\rho}$. Then the cohomology ring $H^{*}\left(F_{\left(k_{1}, \cdots, k_{s}\right)} \times_{\tilde{\rho}} E U(1), \mathbb{C}\right)$ is isomorphic to

$$
\mathbb{C}\left[u_{1}^{1}, \cdots, u_{k_{1}}^{1} ; \cdots ; u_{1}^{s}, \cdots, u_{k_{s}}^{s} ; c_{1}\right] /\left\{\sigma_{1}-\tilde{c}_{1}, \cdots, \sigma_{n}-\tilde{c}_{n}\right\},
$$

where $u_{j}^{i}$ and $\sigma_{j}$ are the same as in Lemma 5.1 and $\tilde{c}_{j}$ is given by $1+\tilde{c}_{1}+$ $\cdots \tilde{c}_{n}=\left(1+r_{1} c_{1}\right) \cdots\left(1+r_{n} c_{1}\right)$.

Note that if $s=2$ and $k_{1}=1$. Under above action we get, after eliminating one parameter,

$$
H_{U(1)}^{*}\left(\mathbb{C} P^{n}\right) \cong \mathbb{C}\left[x, c_{1}\right] /\left\{\left(x-r_{1} c_{1}\right) \cdots\left(x-r_{n} c_{1}\right)\right\}
$$

\subsection{Fiberwise Quantum Cohomology of}

$$
F_{\left(k_{1}, \cdots, k_{s}\right)}(E) \times_{G} E G \rightarrow X \times B G .
$$

Suppose $\mathcal{X} \rightarrow Y$ is a fiber bundle satisfying Assumption ( $\star$ ) and fiber $V$ is a positive symplectic manifold. Assume further that the cohomology ring of $\mathcal{X}$ can be presented by a ring isomorphism

$$
H^{*}(Y)\left[x_{1}, \cdots, x_{t}\right] /\left\{f_{1}, \cdots, f_{s}\right\} \stackrel{p}{\rightarrow} H^{*}(\mathcal{X}, \mathbb{C})
$$


where we view the left side as graded commutative polynomial algebra of $x_{1}, \cdots, x_{t} . p\left(x_{1}\right), \cdots, p\left(x_{t}\right)$ are homogeneous generators of $H^{*}(\mathcal{X} ; \mathbb{C})$, and $f_{1}, \cdots, f_{s}$ are homogeneous polynomials of $x_{1}, \cdots, x_{t}$.

Proposition 5.1. If we give an appropriate degree to $q_{j}$, then there are homogeneous polynomials $g_{i}\left(x_{1}, \cdots, x_{t} ; q_{1}, \cdots, q_{m}\right)$ such that

$$
g_{i}\left(x_{1}, \cdots, x_{t} ; 0, \cdots, 0\right)=0
$$

and $Q H^{*}(\mathcal{X}, Y, \mathbb{C})$ can be presented by a ring isomorphism

$$
H^{*}(Y)\left[x_{1}, \cdots, x_{t} ; q_{1}, \cdots, q_{m}\right] /\left\{f_{1}-g_{1}, \cdots, f_{s}-g_{s}\right\} \stackrel{\tilde{p}}{\rightarrow} Q H^{*}(\mathcal{X}, Y, \mathbb{C}) .
$$

Proof. Let $I$ be an ideal in $\mathbb{C}\left[\left[q_{1}, \cdots, q_{m}\right]\right]$ generated by $q_{1}, \cdots, q_{m}$. Since $V$ is a positive symplectic manifold, for any $\alpha, \beta \in H^{*}(\mathcal{X} ; \mathbb{C})$, we have

$$
\alpha * \beta=\alpha \wedge \beta+h\left(\alpha, \beta ; q_{1}, \cdots, q_{m}\right),
$$

where $h$ is a polynomial in $q_{1}, \cdots, q_{m}$ and $h(\alpha, \beta ; 0, \cdots, 0)=0$. So

$$
\begin{aligned}
Q H^{*}(\mathcal{X}, Y, \mathbb{C})= & \operatorname{span}\left\{p\left(x_{1}\right), \cdots, p\left(x_{t}\right)\right\} \text { use } * \text { multiplication } \\
& +I \cdot Q H^{*}(\mathcal{X}, Y, \mathbb{C}) .
\end{aligned}
$$

By the Nakayama Lemma, it follows

$$
Q H^{*}(\mathcal{X}, Y, \mathbb{C})=\operatorname{span}\left(p\left(x_{1}\right), \cdots, p\left(x_{t}\right)\right) \text { use } * \text { multiplication. }
$$

If $f_{i}\left(x_{1}, \cdots, x_{t}\right)=\sum a_{j_{1}, \cdots, j_{t}}^{i} \cdot x_{1}^{j_{1}} \cdots x_{t}^{j_{t}}$, we denote product $p\left(x_{i}\right) * \cdots *$ $p\left(x_{i}\right)$ ( $j$ factors) by $p\left(x_{i}\right)^{* j}$. Then consider

$$
\begin{aligned}
& \sum a_{j_{1}, \cdots, j_{t}}^{i} \cdot p\left(x_{1}\right)^{* j_{1}} * \cdots * p\left(x_{t}\right)^{* j_{t}} \\
& =\sum a_{j_{1}, \cdots, j_{t}}^{i} \cdot p\left(x_{1}\right)^{j_{1}} \wedge \cdots \wedge p\left(x_{t}\right)^{j_{t}} \\
& \quad+\sum b_{j_{1}, \cdots, j_{t}}^{i}\left(q_{1}, \cdots, q_{m}\right) \cdot p\left(x_{1}\right)^{j_{1}} \wedge \cdots \wedge p\left(x_{t}\right)^{j_{t}}
\end{aligned}
$$

with $b_{j_{1}, \cdots, j_{t}}^{i}(0, \cdots, 0)=0$. By $(5.5)$, we may assume

$$
p\left(x_{1}\right)^{j_{1}} \wedge \cdots \wedge p\left(x_{t}\right)^{j_{t}}=\sum c_{i_{1}, \cdots, i_{t}}^{j_{1}, \cdots, j_{t}} \cdot p\left(x_{1}\right)^{* i_{1}} * \cdots * p\left(x_{t}\right)^{* i_{t}} .
$$

We get

$$
\begin{aligned}
& \sum b_{j_{1}, \cdots, j_{t}}^{i} \cdot p\left(x_{1}\right)^{j_{1}} \cdots p\left(x_{t}\right)^{j_{t}} \\
& =\sum b_{j_{1}, \cdots, j_{t}}^{i}\left(q_{1}, \cdots, q_{m}\right) \cdot c_{i_{1}, \cdots, i_{t}}^{j_{1}, \cdots, j_{t}} \cdot p\left(x_{1}\right)^{* i_{1}} * \cdots * p\left(x_{t}\right)^{* i_{t}} .
\end{aligned}
$$


Let $g_{i}=\sum b_{j_{1}, \cdots, j_{t}}^{i}\left(q_{1}, \cdots, q_{m}\right) \cdot c_{i_{1}, \cdots, i_{t}}^{j_{1}, \cdots, j_{t}} \cdot x_{1}^{i_{1}} \cdots x_{t}^{i_{t}}$. We see that $f_{i}-g_{i}$ is mapped to zero under map $\tilde{p}$. $\tilde{p}$ is a ring homomorphism. Since $p$ is an isomorphism, $\tilde{p}$ is an isomorphism modulo ideal $I$. The fact that $\tilde{p}$ is an isomorphism follows from the Nakayama Lemma. The proposition is proved.

Since we have mathematically defined the equivariant quantum cohomology and established the properties of restriction and induction, the following results from [AS] and [GK] are theorems(see proof in [AS] and [GK]). Some simple matrix manipulation will reduce the matrix in [AS] to the following simpler form.

Theorem 5.1 (Astashkevich-Sadov). The equivariant quantum cohomology of the flag manifold $Q H_{U(n)}^{*}\left(F_{\left(k_{1}, \cdots, k_{s}\right)}, \mathbb{C}\right)$ can be presented as

$$
\begin{aligned}
& \mathbb{C}\left[u_{1}^{1}, \cdots, u_{k_{1}}^{1} ; \cdots ; u_{1}^{s}, \cdots, u_{k_{s}}^{s} ; q_{1}, \cdots, q_{s-1} ; c_{1}, \cdots, c_{n}\right] \\
& \quad /\left\{\sigma_{1}(q)-c_{1}, \cdots, \sigma_{n}(q)-c_{n}\right\},
\end{aligned}
$$

where $c_{i}$ is the $i$-th universal Chern class $c_{i}(U(n))$, the degree of $q_{i}$ is $k_{i}+$ $k_{i+1}$, the degree of $u_{j}^{i}$ is $2 j$, and $\sigma_{i}(q)$ is defined to be the degree $2 i$ part of the expansion of $\operatorname{det}(A)$. The matrix $A$ is defined to be

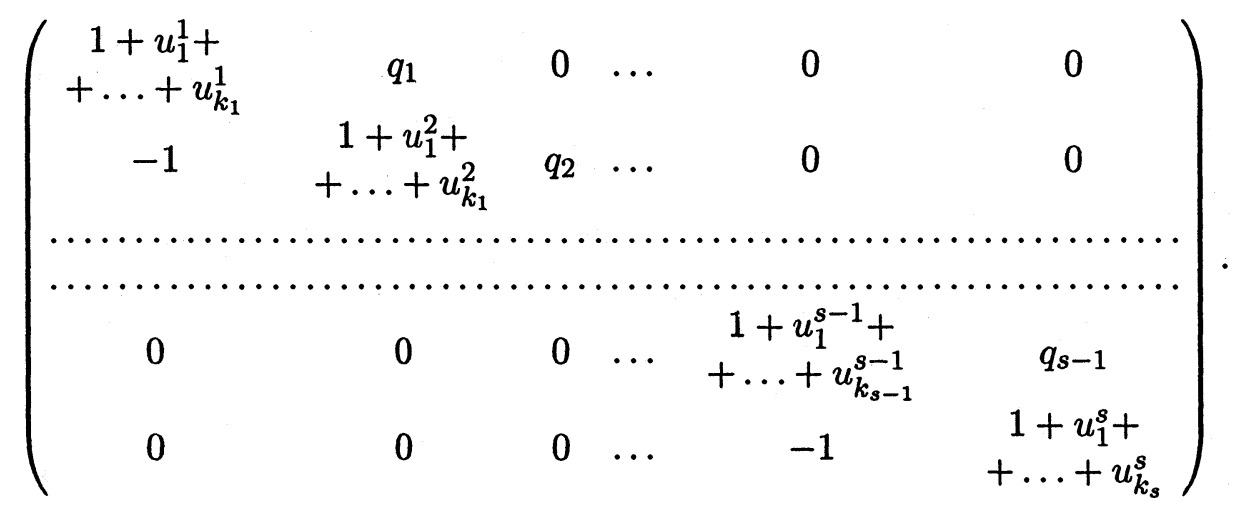

As a special case, when $s=n, k_{1}=\cdots=k_{n}=1$, we have

Theorem 5.2 (Givental-Kim). The equivariant quantum cohomology of the complete flag manifold $Q H_{U(n)}^{*}\left(F_{(1, \cdots, 1)}, \mathbb{C}\right)$ can be presented as

$$
\mathbb{C}\left[u_{1}, \cdots, u_{n} ; q_{1}, \cdots, q_{n-1} ; c_{1}, \cdots, c_{n}\right] /\left\{\sigma_{1}(q)-c_{1}, \cdots, \sigma_{n}(q)-c_{n}\right\},
$$


where $c_{i}$ is the $i$-th universal Chern class, the degree of $q_{i}$ is 2 , the degree of $u_{j}$ is $2 j$, and $\sigma_{i}(q)$ is defined to be the degree $2 i$ part of the expansion of $\operatorname{det}(A)$. The matrix $A$ is defined to be

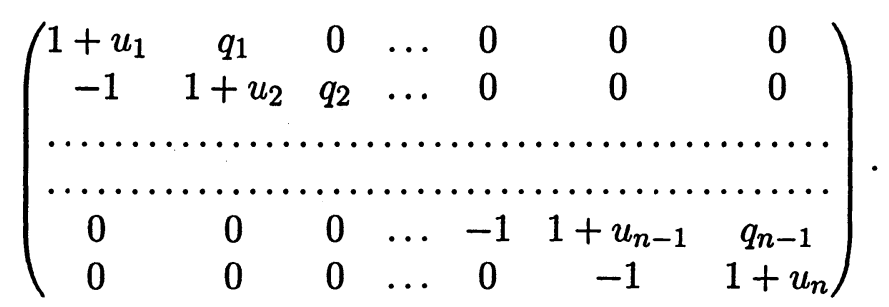

These result can be generalized to

Proposition 5.2. Let $G=U\left(l_{1}\right) \times \cdots \times U\left(l_{t}\right)$, the fiberwise quantum cohomology $Q H^{*}\left(F_{\left(k_{1}, \cdots, k_{s}\right)}(E) \times_{G} E G, X \times B G, \mathbb{C}\right)$ can be presented as

$$
\begin{aligned}
& H^{*}(X, \mathbb{C}) \otimes H^{*}(B G, \mathbb{C})\left[u_{1}^{1}, \cdots, u_{k_{1}}^{1} ; \cdots ; u_{1}^{s}, \cdots, u_{k_{s}}^{s} ; q_{1}, \cdots, q_{s-1}\right] \\
& \quad /\left\{\sigma_{1}(q)-c_{1}\left(p^{*} \tilde{E}\right), \cdots, \sigma_{n}(q)-c_{n}\left(p^{*} \tilde{E}\right)\right\},
\end{aligned}
$$

where $\sigma_{i}(q)$ is defined as in Theorem 5.1 .

Proof. By the Proposition 5.1, we only need to show that relations $\sigma_{i}(q)-c_{i}\left(p^{*} \tilde{E}\right)=0, i=1, \cdots n$, hold in $Q H^{*}\left(F_{\left(k_{1}, \cdots, k_{s}\right)}(E) \times_{G} E G\right.$, $X \times B G, \mathbb{C})$. Choose an $N$ large enough such that $H^{i}(B G) \cong H^{i}\left((B G)_{N}\right)$ for $i=1, \cdots, 2 n+2$. Consider the classifying map $h: X \times(B G)_{N} \rightarrow B(U(n))$ of bundle $\tilde{E}$. $h$ pulls back the universal flag manifold over $B(U(n))$ to $F_{\left(k_{1}, \cdots, k_{s}\right)}(E) \times_{G}(E G)_{N}$. From Theorem 5.1 and the restriction property of the fiberwise quantum cohomology, we conclude that the relation $\sigma_{i}(q)-c_{i}\left(p^{*} \tilde{E}\right)=0$ hold for $i=1, \cdots, n$.

Two special cases are:

Case 1. The fiberwise quantum cohomology

$$
Q H^{*}\left(F_{\left(k_{1}, \cdots, k_{s}\right)} \times_{\rho_{l}} E U(l), B U(l), \mathbb{C}\right)
$$

can be presented as

$$
\begin{aligned}
\mathbb{C} & {\left[u_{1}^{1}, \cdots, u_{k_{1}}^{1} ; \cdots ; u_{1}^{s}, \cdots, u_{k_{s}}^{s} ; c_{1}, \cdots, c_{l} ; q_{1}, \cdots, q_{s-1}\right] } \\
& /\left\{\sigma_{1}(q)-\tilde{c}_{1}, \cdots, \sigma_{n}(q)-\tilde{c}_{n}\right\},
\end{aligned}
$$


where $\sigma_{i}(q)$ is defined as in Theorem 5.1 and $\tilde{c}_{i}$ is defined as in Case 1 in section 5.1.

Case 2. The fiberwise quantum cohomology

$$
Q H^{*}\left(F_{\left(k_{1}, \cdots, k_{s}\right)} \times_{\tilde{\rho}} E U(1), B U(1), \mathbb{C}\right)
$$

can be presented as

$$
\begin{gathered}
\mathbb{C}\left[u_{1}^{1}, \cdots, u_{k_{1}}^{1} ; \cdots ; u_{1}^{s}, \cdots, u_{k_{s}}^{s} ; c_{1} ; q_{1}, \cdots, q_{s-1}\right] \\
/\left\{\sigma_{1}(q)-\tilde{c}_{1}, \cdots, \sigma_{n}(q)-\tilde{c}_{n}\right\},
\end{gathered}
$$

where $\sigma_{i}(q)$ is defined as in Theorem 5.1 and $\tilde{c}_{i}$ and $\tilde{\rho}$ are defined as in Case 2 in section 5.1.

When $s=2, k_{1}=1$, and $\tilde{\rho}$ is the action of $U(1)$ on $\mathbb{C}^{n}$, we have

$$
Q H_{U(1)}^{*}\left(\mathbb{C} P^{n-1} ; \mathbb{C}\right) \cong \mathbb{C}\left[x, c_{1}, q\right] /\left\{\left(x-r_{1} c_{1}\right) \cdots\left(x-r_{n} c_{1}\right)-q^{n}\right\} .
$$

where the degree of $q$ is 2 .

\section{References.}

[AS] A. Astashkevich and V. Sadov, Quantum cohomology of partial flag manifold $F_{n_{1} \cdots n_{k}}$, Comm. Math. Phys. 170 (1995), 503-528.

[B] V. Batyrev, Quantum cohomology of toric manifolds, Astérisque 218 (1992), 9-34.

[BT] R. Bott and L.W. Tu, Differential forms in algebraic topology, SpringerVerlag, New York (1982).

[C] P.E. Conner, Differentiable Periodic Maps, Springer-Verlag, New York (1979).

[CF] I. Ciocan-Fontanine, Quantum cohomology of flag varieties, preprint alggeom/9505002 (1995).

[F1] A. Floer, The unregularized gradient flow of the symplectic action, Comm. Pure Appl. Math. 41 (1988), 775-813.

[GK] A. Givental and B. Kim, Quantum cohomology of flag manifolds and Toda lattices, Comm. Math. Phys. 168 (1995), 609-641.

[JS] J. Jost, Two dimensional geometric variational problems, A Wiley-Interscience Publication, New York (1990). 
[K1] B. Kim, Quantum cohomology of partial flag manifolds and a residue formula for their intersection pairings, IMRN No.1 (1995), 1-16.

[K2] $\longrightarrow$ On equivariant quantum cohomology, preprint q-alg/9509029 (1995).

[M1] D. McDuff, Examples of symplectic structures, Invent. Math. 89 (1987), 1336 .

[M2] The local behaviour of holomorphic curves in almost complex 4-manifolds, J. Diff. Geom. 34 (1991), 143-164.

[M3] Symplectic manifolds with contact boundaries, Invent. Math. 103 (1991), 651-671.

[PW] T. Parker and J. Wolfson, A compactness theorem for Gromov's moduli space, J. Geom. Anal. 3 (1993), 63-98.

[ST] B. Seiber and G. Tian, On quantum cohomology of Fano manifolds and a formula of Vafa and Intriigator, preprint alg-geom/9403010 (1994).

[RT] Y. Ruan and G. Tian, A mathematical theory of quantum cohomology, J. Diff. Geom. 42 (1995), 259-367.

[V] C. Vafa, Topology mirrors and quantum rings, Essays on mirror manifolds, ed S. -T. Yau, International Press (1992).

[W] E. Witten, The Verlinde algebra and the cohomology of Grassmannians, Geometry, Topology, and Physics, ed. S.-T. Yau, International Press (1995), $357-423$.

RECEIVED OCTOBER 16, 1996.

\author{
UNIVERSITY OF MINNESOTA \\ SCHOOL OF MATHEMATICS \\ 127 VINCENT HALL \\ 206 ChURCH ST. S.E. \\ MINNEAPOLIS, MN 55455 \\ E-MAIL ADDRESS: PENGLU@MATH.UMN.EDU
}

